\title{
Comparison of methods for milk pre-processing, exosome isolation, and RNA extraction in bovine and human milk
}

3

Sanoji Wijenayake $^{1 *}$, Shafinaz Eisha ${ }^{1,2}{ }^{*}$, Zoya Tawhidi $^{1}$, Michael A. Pitino ${ }^{5,6}$, Michael A. Steele $^{7}$, Alison S. Fleming ${ }^{8}$, Patrick O. McGowan ${ }^{1,2,3,4}$

*Authors contributed equally to the manuscript

${ }^{1}$ Center for Environmental Epigenetics and Development, Department of Biological Sciences, University of Toronto Scarborough, Toronto. ON, Canada.

${ }^{2}$ Department of Cell and Systems Biology, University of Toronto, Toronto, ON, Canada.

${ }^{3}$ Department of Psychology, University of Toronto, Toronto, ON, Canada.

${ }^{4}$ Department of Physiology, University of Toronto, Toronto, ON, Canada.

${ }^{5}$ Department of Nutritional Sciences, University of Toronto, Toronto, ON, Canada.

${ }^{6}$ Translational Medicine Program, The Hospital for Sick Children, Toronto, ON, Canada

${ }^{7}$ Department of Animal and Poultry Science, University of Guelph, Guelph, ON, Canada.

${ }^{8}$ Department of Psychology, University of Toronto, Mississauga, Mississauga, ON, Canada.

\section{Corresponding Author}

Dr. Patrick O. McGowan

University of Toronto, Scarborough Campus, 1265 Military Trail, Toronto, Ontario, Canada. M1C1A4. Tel: +1-416-208-5153, fax: +1-416-287-7676.

E-mail address: patrick.mcgowan@utoronto.ca

ORCID ID - 0000-0002-6155-490X 


\section{Abstract}

36 Milk is a highly complex, heterogeneous biological fluid that contains bioactive, membrane-

37 bound extracellular vesicles called exosomes. Characterization of milk-derived exosomes

38 (MDEs) is challenging due to the lack of standardized methods that are currently being used for

39 milk pre-processing, exosome isolation, and RNA extraction. In this study, we tested: 1) three

40 pre-processing methods to remove cream, fat, and casein proteins from bovine milk to determine

41 whether pre-processing of whole milk, prior to long-term storage, improves MDE isolations, 2)

42 two commonly-used exosome isolation methods, and 3) four extraction protocols for obtaining

43 high quality MDE RNA from bovine and human milk. MDEs were characterized via

44 Transmission Electron Microscopy (TEM) and Nanoparticle Tracking Analysis (NTA). We also

45 present an optimized method of TEM sample preparation and isolation of total soluble protein

46 from MDEs. Our results indicated that: 1) pre-processing of bovine milk prior to storage does not

47 affect the final exosome yield or the purity, 2) ExoQuick precipitation is better suited for MDE

48 isolation than ultracentrifugation for bovine and human milk, and 3) TRIzol LS produced the

49 highest RNA yield in bovine milk, whereas TRIzol LS, TRIzol+RNA Clean and Concentrator,

50 and TRIzol LS+RNA Clean and Concentrator methods can be used for human milk.

52 Keywords

53 Milk exosomes; bioactive compounds; extracellular vesicles; ExoQuick solution;

54 ultracentrifugation; human; bovine 


\section{Introduction}

56 Maternal milk is the primary nutritional source of newborn mammals. Unlike

57 standardized infant formula, which has a narrow macro/micronutrient range, maternal milk

58 composition is highly dynamic and varies across lactational stages, the circadian cycle, maternal

59 age, ethnicity, diet, and gestational age (1-3). Mammalian milk is a highly complex and

60 heterogeneous solution that contains protein, lipids, carbohydrates, minerals, vitamins, active

61 enzymes, hormones, immune factors, and microbiota $(1,2,4,5)$. Mammalian milk is also

62 biologically customized to fit the physiological, neurodevelopmental, and immune requirements

63 of offspring as they age $(1,2,6,7)$. In particular, human colostrum, is a rich source of

64 immunological components including IgA, lactoferrin, leukocytes, and human milk

65 oligosaccharides (HMOs), and is a vital source of early-life immune programming. In contrast,

66 transition and mature milk are mainly tailored to meet the nutrient and energy demands of the

67 growing offspring $(8,9)$.

68 Recently, maternal milk was found to contain functional microRNAs (miRNAs)

69 encapsulated in protective milk-derived exosomes (MDEs) (10-18). MDEs (a subtype of

70 extracellular vesicles (EVs)) range from 30-150 nm in size and were identified in many

71 mammals including humans, cows, rodents, goats, pigs, and marsupials $(17,19,20)$. MDEs are

72 secreted from mammary gland epithelial cells (MECs), can travel across offspring's intestinal

73 endothelium post-ingestion into circulation, and are taken up by surrounding tissues (14). Upon

74 intake into cells, MDEs may release their cargo and regulate cellular functions of the recipient

75 cells $(10-14,16,17,20-22)$. Lactation-specific miRNAs have been shown to induce post-

76 transcriptional regulation of target mRNA in various recipient tissues $(11,18,20,21,23)$. MDEs

77 have also been shown to cross biological barriers in vitro and in vivo $(14,24)$, increasing interest 
78 in their potential roles in nutritional and agricultural research, translational medicine, and drug 79 therapeutics (25-27).

80 Thus, the isolation and characterization of MDEs have become an important area of

81 research, and there is a need to establish standardized and reproducible methods to isolate and

82 quantify MDEs. In 2014, the International Society for Extracellular Vesicles (ISEV) published a

83 Position Editorial detailing the minimal requirements and recommendations for the identification

84 and characterization of extracellular vesicles and their proposed functions (28). Although these

85 guidelines are regularly updated, they indicate that isolation of intact MDEs with high purity and

86 yield remains a challenge largely due to the numerous handling, processing, and isolation

87 techniques currently used in EV research. The use of non-standardized techniques is more of an

88 issue in MDE research due to the high intra- and interspecific variability that naturally exist

89 across milk samples. In particular, some studies report that changes in temperature and long-term

90 storage of maternal milk do not affect milk composition, integrity, and the final yield of isolated

91 MDEs, while other studies have found that the recovery of MDEs from maternal milk is

92 influenced by sample collection and pre-processing steps (12,29-31). Consequently, here we

93 tested three pre-processing techniques on unpasteurized, whole bovine milk to determine

94 whether removing cream, fat globules and/or casein proteins prior to ultracold storage is required

95 to obtain high quality MDEs.

96 The purity of the isolated exosomes can vary due to the presence of contaminating

97 particles, other EVs, viscosity of the sample, the presence of milk proteins, and nucleic acids that

98 are often precipitated alongside MDEs (32-34). Ultracentrifugation-based (UC) isolations and

99 polymer-based precipitation techniques (i.e. ExoQuick (EQ) reagent) are two of the more

100 commonly used exosome isolation methods (10,11,36-41,12-14,26,32-35). Differential 
101 centrifugation combined with UC is considered the gold standard for exosome isolation,

102 although ultra-high speeds and continuous handling of the samples can result in degradation and

103 low recovery rates. UC is also time consuming, requires specialized equipment and a large

104 starting volume $(26,35)$. In comparison, EQ precipitation has a high recovery rate, is a faster

105 method, does not require a large sample volume $(13,26,32,33,35,37,38)$, and can produce higher

106 miRNA yield with greater purity than other techniques (42). However, EQ precipitation may also

107 co-precipitate contaminants and other EVs that can interfere with downstream applications

$108(26,32,33)$. Therefore, further studies are necessary to confirm the suitability of both isolation

109 techniques in MDE research across species, especially taking into account the downstream

110 applications, including RNA and protein yield resulting from each method. As such, we tested

111 two exosome isolation methods: EQ precipitation and differential UC using unpasteurized

112 bovine and human milk. Transmission electron microscopy (TEM) and Nanoparticle Tracking

113 Analysis (NTA) were used to characterize the isolated exosomes.

114 Since the first identification of RNA in exosomes in 2007 (25), numerous extraction

115 methods have been used for RNA profiling via real time quantitative PCR (RT-qPCR),

116 microarrays, and RNA sequencing. However, enrichment and molecular profiling of MDEs

117 remains technically challenging (37) due to the variability in RNA extraction protocols and

118 commercially available RNA extraction kits that are often utilized in EV research. Here, we

119 tested four MDE-based RNA extraction protocols: three commercially available kits (QIAzol +

120 miRNeasy Mini Kit, TRIzol + RNA Clean and Concentrator Kit (RCC), TRIzol LS + RCC) and

121 an inhouse phenol-based extraction method (TRIzol LS) (43) to identify the most reproducible

122 and optimal RNA extraction method that can be used to generate high quality RNA ( $\geq 17$

123 nucleotides) from bovine and human MDEs. 
124 The overall aim of the current study was to compare methods of milk pre-processing,

125 MDE isolation techniques, and RNA extraction protocols that are currently being used in the EV

126 field to identify the most robust and reproducible techniques that can be used to standardize

127 MDE research. Our results may be useful for the selection of purification methods in future

128 studies using human and/or bovine milk where sample volumes are limited, samples are

129 subjected to extended storage times, and are frozen upon collection (e.g. human donor milk 130 banks).

\section{Materials and Methods}

134 Bovine milk collection and processing

135 Unpasteurized bovine milk was obtained from Loa-De-Mede Holsteins Farm (Oshawa,

136 Ontario, Canada) from 3 different dairy cows. $100 \mathrm{~mL}$ of bovine milk per dairy cow was

137 collected into sterile, DNA/RNase-free conical tubes via hand milking, stored at $4{ }^{\circ} \mathrm{C}$ and

138 transported to the University of Toronto, Scarborough for analysis. All bovine samples were

139 pooled to remove variability in milk composition across dairy cows but, processed separately to

140 ensure independent sample extractions.

141 Three different processing steps were conducted ( $\mathrm{n}=3$ independent trials/group) to test

142 whether removal of cream, fat globules, and casein proteins prior to long-term storage at $-80{ }^{\circ} \mathrm{C}$

143 may impact MDE isolation and characterization efficiency (Supplementary Fig. 1): Group (G) 1)

144 unprocessed, whole milk stored at $-80{ }^{\circ} \mathrm{C}$, where samples were immediately frozen at $-80{ }^{\circ} \mathrm{C}$

145 upon arrival; G2) processed milk without fat globules and cream, where bovine milk was

146 centrifuged twice at $3,000 \mathrm{x} \mathrm{g}$ for $10 \mathrm{~min}$ at room temperature (RT) and the supernatant was

147 collected and stored at $-80{ }^{\circ} \mathrm{C}$; and G3) isolated whey fraction without fat globules, cream,

148 cellular debris, and casein proteins. G3 bovine milk samples were processed as per G2 procedure 
149 plus $2 \mathrm{x}$ centrifugations at $1,200 \mathrm{x}$ g for $10 \mathrm{~min}$ at $4{ }^{\circ} \mathrm{C}$ to remove residual fat globules and

150 cellular debris. Subsequently, the defatted supernatants were centrifuged $2 \mathrm{x}$ at 21,500 x g for 30

$151 \mathrm{~min}$ at $4{ }^{\circ} \mathrm{C}$ followed by a subsequent centrifugation at $21,500 \mathrm{x} \mathrm{g}$ for $1 \mathrm{~h}$ to pellet casein

152 proteins. The supernatants were filtered once through $0.45 \mu \mathrm{M}$ (FroggaBio; SF0.45PES) and

$1530.22 \mu \mathrm{M}$ (FroggaBio; SF0.22PES) PES syringe filters to remove residual cell debris. Isolated

154 whey portion of bovine milk was stored at $-80{ }^{\circ} \mathrm{C}$ for later use.

\section{Human milk collection}

156 Expressed human milk from 2 different donors $(500 \mathrm{~mL} /$ donor) was obtained from the

157 Roger Hixon Ontario Human Milk Bank (Toronto, Ontario, Canada). The unpasteurized samples

158 of human milk used in this study contained a bacterial load $>5 \times 10^{7}$ colony forming units/L and

159 were therefore not suitable for processing or dispensing for human consumption as per the

160 policies of the milk bank. $500 \mathrm{~mL}$ of human milk/donor were collected into sterile collection

161 bags, frozen at $-20{ }^{\circ} \mathrm{C}$ immediately upon collection, remained frozen during transport to the milk

162 bank, and subsequently stored at $-20^{\circ} \mathrm{C}$ till use. Next, the milk samples were thawed overnight at

$1634{ }^{\circ} \mathrm{C}$ and were pooled but processed separately to ensure independent sample extractions. Note:

164 the samples were used in the analysis within 8 months of storage.

\section{Exosome Isolation}

166 Two main isolation methods that are frequently used in exosome-isolation and

167 characterization studies, including EQ precipitation and UC, were compared to determine the

168 most efficient method for isolating MDEs from the whey portion of bovine and human milk $(n=3$

169 independent isolations/method) (Supplementary Fig. 2).

\section{Method 1: ExoQuick Precipitation}


171 Human and G1 bovine milk samples were centrifuged at $2,000 \mathrm{xg}$ for $10 \mathrm{~min}$ at $4{ }^{\circ} \mathrm{C}$ to

172 remove upper cream layer. The supernatants were collected carefully and centrifuged again at

$17312,000 \times \mathrm{g}$ for $30 \mathrm{~min}$ at $4{ }^{\circ} \mathrm{C}$ to remove fat cells and globules. Finally, the supernatants were

174 further centrifuged at $12,000 \mathrm{x}$ f for $5 \mathrm{~min}$ at $4{ }^{\circ} \mathrm{C}$ to pellet cell debris. Supernatants were

175 isolated and filtered once through $0.45 \mu \mathrm{M}$ syringe filters to eliminate traces of cellular debris.

$176 \mathrm{G} 2$ bovine samples were filtered once through $0.45 \mu \mathrm{M}$ (FroggaBio; SF0.45PES) syringe filters

177 to eliminate cellular debris. G3 whey fractions were used directly in the exosome precipitation

178 reaction.

179 MDEs were isolated from G1-G3 bovine and human milk samples (1.5 mL/sample) using

180 the EQ reagent (System Biosciences: EXOQ5A-1) $(13,14,38,40,44)$. EQ reagent was added to all

181 samples (1: $0.2, \mathrm{v} / \mathrm{v})$, mixed by inversion, and incubated for $12 \mathrm{~h}$ at $4{ }^{\circ} \mathrm{C}$ to enhance

182 precipitation. Post incubation, all samples were centrifuged at $18,000 \mathrm{x} \mathrm{g}$ for $45 \mathrm{~min}$ at $4{ }^{\circ} \mathrm{C}$ to

183 pellet the exosomes. The pelleted exosomes were re-suspended in 200-400 $\mu \mathrm{L}$ of 1x-filtered PBS

184 (determined based on the size of the pellet). The supernatants were used as the negative control

185 for subsequent experiments.

186 Method 2: Ultracentrifugation

187 An optimized version of the UC method as previously described $(10,11,35,39,40,45)$ was

188 used. Human and G1 bovine milk samples were centrifuged twice at 3,000 x g for $10 \mathrm{~min}$ at RT

189 to remove the upper cream layer. The supernatants were centrifuged twice at $1,200 \times \mathrm{g}$ for 10

$190 \mathrm{~min}$ at $4{ }^{\circ} \mathrm{C}$, followed by two top-speed centrifugations at $21,500 \mathrm{x} \mathrm{g}$ for $30 \mathrm{~min}$ at $4{ }^{\circ} \mathrm{C}$ and a

191 final centrifugation at $21,500 \mathrm{x}$ for $1 \mathrm{~h}$ at $4{ }^{\circ} \mathrm{C}$ to remove fat globules and casein proteins. The

192 supernatants were filtered through $0.45 \mu \mathrm{M}$ and $0.22 \mu \mathrm{M}$ PES syringe filters to remove cell

193 debris and residual fat cells. G2 bovine samples were centrifuged twice at 1,200 x g for $10 \mathrm{~min}$ at 
$1944{ }^{\circ} \mathrm{C}$, followed by two top-speed, centrifugations at $21,500 \times \mathrm{g}$ for $30 \mathrm{~min}$ at $4{ }^{\circ} \mathrm{C}$ and a final

195 centrifugation at $21,500 \mathrm{x}$ g for $1 \mathrm{~h}$ at $4{ }^{\circ} \mathrm{C}$ to remove casein proteins. Similar to G1 bovine

196 samples, the supernatants were filtered through $0.45 \mu \mathrm{M}$ and $0.22 \mu \mathrm{M}$ PES syringe filters to

197 remove cell debris and residual fat cells. G3 bovine samples were directly used in the

198 ultracentrifugation step. All whey fractions were centrifuged at 100,000 x g for 90 min using a

199 SW55 TI swing bucket ultracentrifuge at $4{ }^{\circ} \mathrm{C}$. The pellets were re-suspended in $200 \mu \mathrm{L}$ of

200 filtered $1 \mathrm{X}$ PBS. The supernatants were used as the negative control for all subsequent

201 experiments.

\section{Exosome Visualization}

203 The isolated MDEs were visualized by TEM with negative staining using an optimized

204 sample preparation technique. Four-hundred mesh carbon-coated copper grids (Electron

205 Microscopy Sciences; CF400-CU-50) were incubated for 5 min with $10 \mu \mathrm{L}$ of isolated MDEs.

206 Three consecutive wash steps with $20 \mu \mathrm{L}$ of $\mathrm{ddH}_{2} \mathrm{O}$ (2 min each) were done to minimize

207 crystallization and coagulation of milk residue. All copper grids were negatively stained with 20

$208 \mu \mathrm{L}$ of $2 \%$ uranyl acetate for $5 \mathrm{~min}$ at RT (Supplementary Fig. 3). All excess reagents were

209 removed with filter paper to ensure a $100 \mathrm{~nm}$ thickness and all grids were dried under an

210 incandescent light for 2 min. The copper grids were observed and photographed using a Hitachi

211 H-7500 transmission electron microscope with a Megaview III camera (Olympus).

\section{Exosome Quantification}

213 Particle size and concentration of isolated exosomes and negative controls were

214 quantified using Nanoparticle Tracking Analysis (Malvern Instruments Ltd.; NanoSight NS300)

215 as per manufacturer's instructions at the Structural and Biophysical Core Facility located in the

216 Hospital for Sick Children (Toronto, Ontario, Canada). NTA utilize the properties of Brownian 
217 motion and light scattering to measure particle size and concentration (particles/mL) of EVs. The

218 software tracks individual particles frame by frame and calculates particle size based on Stokes-

219 Einstein equation (46). A 1:700 dilution factor for bovine samples and a 1:300 dilution factor for

220 human samples were used for the analysis. Standard curves ranging from 1:100 to 1:700 (v/v in

221 1X PBS) were run per species to determine the correct dilution range (60-100 particles/frame).

222 An absolute control of 1X PBS was also assessed. Processing settings consisted of a detection

223 threshold of 8 , camera level of 15 , and 3 replicates of 30 s captures. The laser type was Blue 488

$224 \mathrm{~nm}$.

\section{RNA Extraction of MDEs}

226 Four RNA extraction methods that are commonly used in exosome studies (7$22712,18,20,27,32,34,35,37,39,41,43-46)$ were compared to identify the most repeatable and

228 suitable method to obtain high quality MDEs from bovine and human milk ( $\mathrm{n}=6$ independent 229 extractions/protocol) (Supplementary Fig. 4).

\section{Method 1: QIAzol + miRNeasy Mini Kit}

231 QIAzol lysis reagent combined with miRNeasy Mini Kit (Qiagen; 217004) was used as

232 per the manufacturer's instructions with slight modifications. QIAzol reagent was added to 200

$233 \mu \mathrm{L}$ of isolated milk exosomes $(5: 1, \mathrm{v} / \mathrm{v})$. All samples were homogenized by pipetting $20 \mathrm{x}$

234 followed by aspirating $20 \mathrm{x}$ with 18-gauge needles and incubated for $5 \mathrm{~min}$ at RT. Chloroform

235 was added to each sample (1:1, v/v to the starting sample), then all samples were shaken

236 vigorously for $15 \mathrm{~s}$ to $\mathrm{mix}$ and incubated for $3 \mathrm{~min}$ at RT. Phase separation was done by

237 centrifuging at $12,000 \times \mathrm{g}$ for $15 \mathrm{~min}$ at $4{ }^{\circ} \mathrm{C}$. The upper aqueous phase was collected for RNA

238 extraction and $100 \%$ ethanol (1.5:1, v/v) was added and pipetted to mix. The lower phenol layer

239 was kept aside for protein extractions. All content, including any precipitate, was transferred to 
240 RNeasy mini columns and centrifuged at $10,000 \mathrm{x} \mathrm{g}$ for $15 \mathrm{~s}$ at RT. Columns were washed

241 according to the manufacturer's instructions. Post washing, columns were centrifuged at the

242 maximum speed for $5 \mathrm{~min}$ to remove ethanol contamination. RNA was eluted with $50 \mu \mathrm{L}$

243 DNA/RNase free $\mathrm{ddH}_{2} \mathrm{O}$. Columns were incubated for $10 \mathrm{~min}$ after adding DNA/RNase-free

244 water and re-eluted to increase RNA yield. RNA concentration (ng/ $\mu \mathrm{L})$ and quality (A260/A280;

245 A260/A230) were determined using a Nanodrop Spectrophotometer (Thermo Scientific; ND246 2000C).

\section{Method 2: TRIzol LS}

248 TRIzol LS reagent (Thermo Scientific; 10296010) was used as described in (43) with

249 minor modifications. TRIzol LS reagent was added to $200 \mu \mathrm{L}$ of isolated milk exosomes (3:1, $250 \mathrm{v} / \mathrm{v})$. All samples were homogenized by pipetting $20 \mathrm{x}$ followed by aspirating $20 \mathrm{x}$ with 18 -gauge

251 needles and incubated for $5 \mathrm{~min}$ at RT. $200 \mu \mathrm{L}$ of chloroform was added to each sample.

252 Samples were mixed by shaking for $30 \mathrm{~s}$ and incubated for $10 \mathrm{~min}$ at RT. Phase separation was

253 done by centrifuging samples at $12,000 \mathrm{x}$ g for $15 \mathrm{~min}$ at $4{ }^{\circ} \mathrm{C}$. The upper aqueous phase was

254 collected for RNA extraction, while the lower phenol phase was kept aside for protein isolation.

$25510 \%$ sodium acetate $(3 \mathrm{M}, \mathrm{pH} 5.5), 4 \mu \mathrm{L}$ of glycogen, and $100 \%$ ethanol $(2.5: 1, \mathrm{v} / \mathrm{v})$ of the

256 volume of aqueous phase were added per sample. Samples were mixed and incubated overnight

257 at $-80{ }^{\circ} \mathrm{C}$ to facilitate RNA precipitation. Post incubation, samples were centrifuged at $16,000 \mathrm{x} \mathrm{g}$

258 for $30 \mathrm{~min}$ at $4{ }^{\circ} \mathrm{C}$ to pellet the RNA. Subsequently, RNA pellets were washed with $500 \mu \mathrm{L}$ of 70

$259 \%$ ethanol and centrifuged at $16,000 \mathrm{xg}$ for $5 \mathrm{~min}$ at $4{ }^{\circ} \mathrm{C}$. Ethanol was aspirated and pellet was

260 centrifuged again at top speed for $1 \mathrm{~min}$ to remove any residual ethanol. Of note, that this step

261 was extremely important for the proper removal of ethanol contamination. The pellet was air262 dried for 10 min and re-suspended in $32 \mu \mathrm{L}$ of RNase-free $\mathrm{ddH}_{2} \mathrm{O}$. RNA concentration $(\mathrm{ng} / \mu \mathrm{L})$ 
263 and quality (A260/A280; A260/A230) were determined using a Nanodrop Spectrophotometer

264 (Thermo Scientific; ND-2000C).

265 Method 3: TRIzol + RNA Clean and Concentrator Kit

266 MDEs were lysed using TRIzol reagent (Thermo Scientific; 15596026) as per the 267 manufacturer's instructions with modifications. Cold TRIzol reagent was added to $200 \mu \mathrm{L}$ of 268 isolated MDEs (5:1, v/v). Samples were homogenized by pipetting $20 \mathrm{x}$ followed by aspirating $26920 \mathrm{x}$ with 18-gauge needles and incubated for $5 \mathrm{~min}$ at RT. Subsequently, $200 \mu \mathrm{L}$ of chloroform 270 was added to the samples (0.2:1, v/v to TRIzol), vortexed for $30 \mathrm{~s}$, and incubated for $3 \mathrm{~min}$ at 271 RT. Samples were centrifuged at $12,000 \mathrm{x} \mathrm{g}$ for $15 \mathrm{~min}$ at $4{ }^{\circ} \mathrm{C}$ to induce phase separation. The 272 colorless, upper aqueous phase, containing total soluble RNA, was collected while the lower 273 phenol phase was kept aside for protein isolation. Following TRIzol phase separation, $100 \%$ 274 ethanol was added to the aqueous phase $(1: 1, \mathrm{v} / \mathrm{v})$ and transferred to RNA Clean and 275 Concentrator (RCC) ${ }^{\mathrm{TM}}-5$ kit (Zymo Research; R1013) and centrifuged at 16,000 x g for $30 \mathrm{~s}$. 276 RCC kit can be used to isolate ultra-pure, total RNA ( $\geq 17$ ntd in length). Subsequently, the 277 columns were washed once with RNA prep buffer and twice with RNA wash buffer (supplied 278 with the kit) as per the manufacturer's instructions. After the last wash, the columns were 279 centrifuged on max speed for 5 min to remove residual ethanol. RNA was eluted with $40 \mu \mathrm{L}$ of 280 DNA/RNase-free water. Note: columns were incubated for 10 min after adding DNA/RNase-free 281 water and re-eluted to increase RNA yield. RNA concentration (ng/ $\mu \mathrm{L})$ and quality (A260/A280; 282 A260/A230) were determined using a Nanodrop Spectrophotometer (Thermo Scientific; ND283 2000C).

284 Method 4: TRIzol LS + RNA Clean and Concentrator Kit 
MDEs were lysed using TRIzol LS reagent (Thermo Scientific; 10296010) as per the

286 manufacturer's instructions with modifications. Cold TRIzol LS reagent was added to $200 \mu \mathrm{L}$ of

287 isolated milk exosomes $(3: 1, \mathrm{v} / \mathrm{v})$. Samples were homogenized by pipetting $20 \mathrm{x}$ followed by 288 aspirating $20 \mathrm{x}$ with 18 -gauge needles and incubated for $5 \mathrm{~min}$ at RT. Subsequently, $200 \mu \mathrm{L}$ of 289 chloroform was added to the samples (0.3:1, v/v to TRIzol LS), vortexed for $30 \mathrm{~s}$ and incubated 290 for $3 \mathrm{~min}$ at RT. Samples were centrifuged at $12,000 \mathrm{xg}$ for $15 \mathrm{~min}$ at $4{ }^{\circ} \mathrm{C}$ to induce phase 291 separation. The colorless, upper aqueous phase, containing total soluble RNA was collected 292 while the lower phenol phase was kept aside for protein isolation. All the steps involving the use 293 of RCC kit are identical to that of TRIzol + RCC method. RNA concentration (ng/ $\mu \mathrm{L})$ and quality 294 (A260/A280; A260/A230) were determined using a Nanodrop Spectrophotometer (Thermo 295 Scientific; ND-2000C).

All RNA samples were separated via gel electrophoresis (1\% TAE agarose, w/v) for 60

$297 \mathrm{~min}$ at $300 \mathrm{mV}$ to visualize RNA integrity and traces of cellular RNA contamination of the 298 isolated exosome fractions. $1 \mathrm{kB}$ DNA ladder (250 bp - 4,000 bp) (Genedirex; DM101-R500) 299 and a cellular RNA control were run alongside the samples.

\section{$300 \quad$ Protein Isolation}

301 Total soluble protein was isolated from the lower phenol phase from all four RNA 302 extraction protocols listed above, as per manufacturer's instructions with modifications. $100 \%$ 303 ethanol was added (1:0.3, v/v) to the samples, incubated for $3 \mathrm{~min}$ at RT, and centrifuged at $3042,000 \mathrm{x} \mathrm{g}$ for $5 \mathrm{~min}$ at $4{ }^{\circ} \mathrm{C}$ to pellet gDNA. Isopropanol $(1: 1.5, \mathrm{v} / \mathrm{v})$ was added to the resulting 305 supernatant and incubated for $10 \mathrm{~min}$ at RT. Subsequently, the samples were centrifuged at $30612,000 \mathrm{x} \mathrm{g}$ for $10 \mathrm{~min}$ at $4^{\circ} \mathrm{C}$ to pellet the proteins. The pellets were washed with $0.3 \mathrm{M}$ 307 guanidine hydrochloride in $95 \%$ ethanol (1:2, v/v) and incubated for $20 \mathrm{~min}$ at RT. The washing 
308 step was repeated twice more. Finally, the pellets were washed with $2 \mathrm{~mL}$ of $100 \%$ ethanol and

309 incubated for another $20 \mathrm{~min}$ at RT. All centrifugation steps were done at 7,500 x $\mathrm{g}$ for $5 \mathrm{~min}$ at

$3104{ }^{\circ} \mathrm{C}$. The pellets were air-dried for $10 \mathrm{~min}$ to remove residual ethanol and phenol contamination.

311 The pellets were re-suspended in $200 \mu \mathrm{L}$ of $1 \%$ SDS and incubated in a water bath at $50{ }^{\circ} \mathrm{C}$ for

$31220 \mathrm{~min}$. To enhance the solubility of proteins, the samples were incubated for $12 \mathrm{~h}$ at $4{ }^{\circ} \mathrm{C}$ and

313 centrifuged at $10,000 \mathrm{x}$ g for $10 \mathrm{~min}$ at $4{ }^{\circ} \mathrm{C}$ to remove insoluble material. Protein concentration

314 was determined using a Pierce ${ }^{\mathrm{TM}}$ Bicinchoninic Acid (BCA) Protein Microplate Assay (Thermo

315 Scientific; 23225) and BSA standards ranging from $2000 \mu \mathrm{g} / \mathrm{mL}$ to $0 \mu \mathrm{g} / \mathrm{mL}$.

\section{Statistical Analysis}

317 Statistical analysis was conducted using SPSS statistical software (IBM Corp.), and 318 figures were created using GraphPad Prism Version 7 and BioRender.com. A Shapiro-Wilk test 319 was used to assess normality. The data were normally distributed ( $>0.05)$ and as such 320 parametric analyses were carried out. One-way analysis of variance (ANOVA) was used to test 321 for main effect of bovine milk pre-processing (G1-G3). Three-way ANOVA was used to test for 322 main effect of exosome fractionation (pellet and supernatant), exosome isolation methods (EQ 323 and UC), RNA extraction protocols, and their interactions. Tukey post-hoc analysis was used to 324 conduct all pairwise comparisons. Relationships were considered statistically significant at $\mathrm{p} \leq$ 3250.05 .

\section{$327 \quad$ Results}

\section{Pre-processing of Bovine Milk}

329 NTA (Fig 1A-B), RNA (Fig. 1C-E), and protein (Fig. 1F) results indicated no significant

330 effect of pre-processing on bovine milk samples (NTA: main effect of pre-processing, $\left(F_{(2,23)}=\right.$ 
$3310.440, p=0.654)$; RNA concentration: $\left(F_{(2,95)}=1.050, p=0.354\right)$; RNA purity-A260/A280:

$332 \quad\left(F_{(2,95)}=1.313, p=0.274\right) ; \mathrm{A} 260 / \mathrm{A} 230:\left(F_{(2,95)}=0.568, p=0.569\right) ;$ protein concentration: $\left(F_{(2,}\right.$

$33395)=0.431, p=0.651)$. However, slight differences in exosome quality were visually observed

334 across G1-G3 via TEM (Fig. 2-3).

335 Bovine Milk-derived Exosome Isolation: EQ versus UC Methods

336 Bovine exosome pellets were compared against their corresponding supernatants

337 (supernatants were used in our study as a negative control that should not contain MDEs) to test

338 for efficiency of fractionation. According to the TEM analysis, both methods yielded more

339 MDEs of the correct EV size $(30-150 \mathrm{~nm})$ in the pellet fractions, when compared to their

340 respective supernatants. Similarly, NTA indicated that exosome pellets isolated via the EQ

341 method contained more MDEs [particles/mL], belonging to the correct particle range, when

342 compared to the negative controls (main effect of fractionation, $\left(F_{(1,23)}=22.236, p<0.001\right.$ ),

343 Tukey post hoc $p<0.001$, Fig. 4). However, the MDE concentration between the UC pellet and

344 the supernatant remained unchanged in bovine milk (Tukey post hoc $p=0.995$ ). Additionally,

345 EQ and UC exosome pellets resulted in higher RNA yield compared to their corresponding

346 supernatants (main effect of fractionation, RNA concentration: $\left(F_{(1,95)}=26.756, p<0.001\right.$ );

347 A260/A280: $\left(F_{(1,95)}=13.244, p<0.001\right)$; A260/A230: $\left(F_{(1,95)}=46.868, p<0.001\right)$. Similar

348 results were seen in gel electrophoresis, where exosome pellets extracted from both EQ and UC

349 had visible RNA bands (ranging from 20-150 bp) compared to their respective supernatants (Fig.

350 5). There was also a significant difference in total soluble protein $[\mu \mathrm{g} / \mathrm{mL}]$ between exosome

351 pellets and their respective supernatants $\left(F_{(1,95)}=69.884, p<0.001\right.$, Fig 6)).

352 EQ versus UC isolation methods were compared to determine whether one method is

353 more suitable than the other for isolating high quality, intact MDEs. According to TEM results, 
354 exosome pellets isolated via UC contained more intact, spherical exosomes of the correct particle

355 size (30-150nm) compared to the EQ pellets. However, the NTA results indicated that EQ pellets

356 contained a higher concentration of exosomes of the correct particle size compared to UC (main

357 effect of exosome isolation method, $\left.\left(F_{(1,23)}=11.942, p<0.002\right)\right)$. Minimal differences were seen

358 in RNA concentration, RNA purity (A260/A80; A260/A230), and protein concentration $[\mu \mathrm{g} / \mathrm{mL}]$

359 of exosome pellets obtained via EQ and UC methods (main effect of exosome isolation method,

360 A260/A280: $\left(F_{(1,95)}=1.186, p=0.279\right) ;$ A260/A230: $\left(F_{(1,95)}=0.629, p=0.430\right)$; and protein

361 concentration: $\left.\left(F_{(1,95)}=0.814, p=0.370\right)\right)$.

\section{RNA Extraction of Bovine Milk-derived Exosomes}

363 The TRIzol LS protocol produced the highest RNA yield $[\mathrm{ng} / \mu \mathrm{L}]$ for bovine MDEs

364 isolated via EQ and UC methods. EQ: TRIzol LS vs QIAzol miRNeasy minikit (main effect of 365 RNA extraction protocol, $\left(F_{(3,95)}=51.215, p<0.001\right)$, Tukey post hoc $\left.p=0.002\right)$, TRIzol LS vs

366 TRIzol + RCC (Tukey post hoc $p<0.001$ ), and TRIzol LS vs TRIzol LS + RCC (Tukey post 367 hoc $p<0.001)$. UC: TRIzol LS vs QIAzol miRNeasy minikit (Tukey post hoc $p<0.001$ ), TRIzol

368 LS vs TRIzol + RCC (Tukey post hoc $p<0.001)$, and TRIzol LS vs TRIzol LS + RCC (Tukey

369 post hoc $p<0.001)$. There was also a significant fractionation/RNA extraction interaction $\left(F_{(3,95)}\right.$ $370=9.569, p<0.001)$.

371 RNA purity (A260/A280) showed a significant main effect of RNA extraction protocol, $372\left(F_{(3,95)}=29.122, p<0.001\right)$ and fractionation/RNA extraction interaction, $\left(F_{(3,95)}=6.637, p<\right.$ 373 0.001). Moreover, TRIzol + RCC and TRIzol LS + RCC protocols produced higher quality RNA 374 (A260/A230) compared to QIAzol miRNeasy minikit and TRIzol LS protocols. UC exosome 375 pellets: TRIzol + RCC vs QIAzol miRNeasy minikit (main effect of RNA extraction protocol, $376\left(F_{(3,95)}=16.805, p<0.001\right)$, Tukey post hoc $\left.p=0.001\right)$, TRIzol + RCC vs TRIzol LS (Tukey 
377 post hoc $p<0.001)$. EQ exosome pellets: TRIzol LS + RCC vs QIAzol miRNeasy minikit

378 (Tukey post hoc $p=0.027$ ), and TRIzol LS + RCC vs TRIzol LS (Tukey post hoc $p=0.003$ ).

379 Total soluble protein $[\mu \mathrm{g} / \mathrm{mL}]$ isolated from the lower organic-phase of the four phenol-

380 based RNA extractions remained unchanged across methods (main effect of RNA extraction $\left.381 \operatorname{protocol}\left(F_{(3,95)}=1.416, p=0.244\right)\right)$.

\section{Human Milk-derived Exosome Isolation: EQ versus UC Methods}

384 Human MDEs isolated via EQ and UC methods were compared against their 385 corresponding supernatants to determine the efficiency of fractionation. TEM results confirmed 386 that EQ and UC pellets contained more intact exosomes of the correct MV size compared to their 387 respective supernatants (Fig. 7). NTA analysis also indicated that exosome pellets isolated via 388 both EQ and UC methods produced more exosomes [particles/mL] compared to their 389 corresponding supernatants (main effect of fractionation, $\left(F_{(1,7)}=534.670, p<0.001\right.$ ), Tukey 390 post hoc $p<0.001$, Fig. 8). Similarly, RNA concentration $[\mathrm{ng} / \mu \mathrm{L}]\left(F_{(1,31)}=131.638, p<0.001\right)$ 391 and RNA purity (A260/A280 $\left(F_{(1,31)}=154.293, p<0.001\right) ; \mathrm{A} 260 / \mathrm{A} 230\left(F_{(1,31)}=111.250, p<\right.$ 392 0.001)) of the exosome pellets were higher than their respective supernatants. Corresponding 393 results were seen in the 1\% TAE agarose gel, where exosomes pellets isolated via EQ and UC 394 methods had visible RNA bands (ranging from 20-200 base pairs) compared to their supernatants 395 (Fig. 9). Total soluble protein concentration $[\mu \mathrm{g} / \mathrm{mL}]$ was also higher in the pellet fractions $\left(F_{(1,}\right.$ $39631)=28.778, p<0.001$, Fig. 10) compared to the supernatants.

397 According to the TEM, the EQ pellets contained less cell debris and more intact 398 exosomes within the expected size range $(30-150 \mathrm{~nm})$ compared to the UC exosome pellets. 399 However, the NTA analysis indicated no differences in exosome concentration [particles/mL] 
400 between EQ and UC isolation methods (main effect of exosome isolation method, $\left(F_{(1,7)}=0.848\right.$, $401 p=0.409)$ ). EQ exosome pellets contained more RNA $[\mathrm{ng} / \mu \mathrm{L}]$ (main effect of exosome isolation 402 method, $\left.\left(F_{(1,31)}=39.609, p<0.001\right)\right)$, and total soluble protein $[\mu \mathrm{g} / \mathrm{mL}]\left(F_{(1,31)}=5.220, p=\right.$ 403 0.036) than UC exosome pellets. There was a significant fractionation/exosome isolation 404 interaction (RNA concentration: $\left(F_{(1,31)}=40.938, p<0.001\right)$; A260/A280: $\left(F_{(1,31)}=7.877, p=\right.$ 405 0.013); and A260/A230: $\left.\left(F_{(1,31)}=14.082, p=0.002\right)\right)$.

\section{RNA Extraction of Human Milk-derived Exosome}

411 0.063), and TRIzol LS + RCC (Tukey post hoc $p=0.049$ ). There was a significant

412 fractionation/RNA extraction interaction $\left(F_{(3,31)}=7.286, p=0.003\right)$ and exosome isolation/RNA 413 extraction interaction $\left(F_{(3,31)}=4.855, p=0.014\right)$. There was also significant main effect of RNA 414 extraction protocol on RNA purity (A260/A280: $\left(F_{(3,31)}=9.168, p=0.001\right)$; A260/A230: $\left(F_{(3,31)}\right.$ $415=6.039, p=0.006))$ and protein concentration: $\left(F_{(3,31)}=6.413, p=0.005\right)$.

\section{Discussion}

Recent studies have identified a number of novel bioactive components in maternal milk,

420 including lactation-specific miRNAs and stem cells. The abundance of lactation-specific 421 miRNAs in milk fractions $(17,18,21,50)$, their stability in offspring's GI tracts $(11,13,16,41)$, and 422 their localization in peripheral organs (14), the blood (19), and the brain (24), suggest an 423 important role for maternal milk miRNAs in postnatal development. Reproducible and 424 standardized methods for milk processing, storage, and MDE isolation are required to ensure 
425 accurate characterization of MDEs and their function. In this study, we tested three milk pre-

426 processing methods, two commonly used MDE isolation techniques, and four RNA extraction

427 protocols used to obtain high quality exosomal RNA from bovine and human milk. Collectively,

428 our results indicate that pre-processing of whole milk to remove fat, cream, and casein proteins

429 prior to long-term storage is not required to obtain high-quality MDEs. Moreover, EQ

430 precipitation is better suited for the isolation of exosomes from bovine and human whey milk

431 fractions compared to UC. Further, the TRIzol LS protocol produced the highest RNA yield from

432 bovine MDEs, while TRIzol LS, TRIzol+RCC, and TRIzol LS+RCC methods were found to be

433 efficient for the extraction of high quality human MDEs.

434 Pre-processing of Bovine Milk

435 Some studies have reported that the pre-processing of whole milk to remove 436 contaminants and unwanted components prior to long-term storage affects the yield and the 437 purity of isolated MDEs $(29,31)$, while other studies have reported minimal effects of pre438 processing on the quality of MDEs $(12,51,52)$. We found some differences in exosome quality 439 among the three pre-processing groups via TEM. However, NTA, RNA, and protein data 440 confirmed that the pre-processing of milk prior to long-term storage at $-80{ }^{\circ} \mathrm{C}$ is not necessary to 441 obtain high quality MDEs (Fig. 1). The visual differences in TEM images that were recorded 442 across G1-G3 are not surprising given that operator image selection can lead to false 443 interpretations regarding EV quality. As others have shown, operator image selection is more 444 suitable to demonstrate the presence of EVs in a sample, but is less suitable to demonstrate the 445 quality of the fractionation (29). Our results are thus consistent with other published studies 446 indicating that pre-processing of whole milk prior to long-term storage does not affect the final 447 MDE yield, integrity and/or biological activities (51,52). Further, hold pasteurization involving 
$44862.5^{\circ} \mathrm{C}$ temperature treatment for $30 \mathrm{~min}$ also does not appear to adversely affect the integrity

449 and biological function of MDEs, and pasteurized MDEs were shown to be as functionally

450 beneficial as raw milk MDEs in therapeutics (12). However, a few studies have shown the

451 opposite results, where pre-processing of whole milk prior to storage was required for successful

452 MDE isolations $(29,30,51)$. Notably, these select studies used different storage conditions,

453 handling procedures, sample preparation, and exosome isolation techniques compared to the

454 methods used in our study. In particular, Zonneveld et al. (2014) processed a batch of human

455 milk immediately after collection, processed two more batches after storing at $-80{ }^{\circ} \mathrm{C}$ and $4{ }^{\circ} \mathrm{C}$

456 for $2 \mathrm{~h}$, and used density gradient centrifugation for isolating MDEs (29). In contrast, the

457 samples of human donor milk used in our study were obtained from a human milk bank and it

458 was stored immediately upon collection at $-20{ }^{\circ} \mathrm{C}$ and thawed at $4{ }^{\circ} \mathrm{C}$ overnight before

459 processing. Note: the standard procedure for the storage of human milk by milk banks is that all

460 human milk samples are frozen immediately upon collection by the donors at $-20^{\circ} \mathrm{C}$, transported

461 to the milk bank in a frozen state, and subsequently stored at $-20{ }^{\circ} \mathrm{C}$ until ready for pasteurization

462 and processing. As such, differences in sample handling conditions limit direct comparisons

463 between these studies and the present investigation. This highlights one of the main limitations

464 of current MDE research, where a lack of standardized methods for sample handling/storage and

465 MDE isolations may lead to erroneous conclusions and incomparable results. Our findings

466 suggest that it is feasible to process whole milk after long-term storage without incurring

467 significant losses in MDE concentration, purity, and integrity. This is a very useful finding as all

468 donor milk obtained from human milk banks is frozen soon after expression without pre-

469 processing. As a majority of MDE research in human milk involves samples obtained from milk

470 banks, the ability to isolate high quality MDEs in large quantities post-long-term storage is of 
471 great value. In addition, there is evidence that eliminating time sensitive pre-processing

472 requirements may also increase the viability of other bioactive and macro/micronutrient

473 components of milk, including hormones, vitamins, and growth factors that are sensitive to

474 repeated handling and temperature fluctuations $(29,31)$.

\section{Bovine Milk-derived Exosome Isolation: UC versus EQ Methods}

476 UC-based isolations, EQ precipitation, size-based isolations, and immunity capture-based

477 techniques are some of the most commonly used exosome isolation methods. Each technique

478 utilizes a particular trait of EVs, including density, shape, size, and/or surface

479 receptors/membrane proteins for the isolations. As such, there are unique advantages and

480 disadvantages to each method (33). A robust method with the ability to minimize exosome loss,

481 lysis, as well as co-isolation of contaminants is an important pre-requisite that should be

482 standardized across $\mathrm{MDE}$ research in order to minimize confounding effects that can alter

483 downstream applications. Density gradient centrifugation, where exosomes are separated based

484 on size, mass, and density in a sucrose gradient, is the gold standard for exosome isolations.

485 However, an important drawback of this technique is the loading limitation, where only a small

486 volume of milk must be loaded onto the gradient (33). Density gradient centrifugation can also

487 be labor-intensive and can take more than $24 \mathrm{~h}$ to complete (53). Thus, differential UC (100,000

$488 \mathrm{x} \mathrm{g}$ for $90 \mathrm{~min}$ ) coupled with serial filtration has become increasingly popular for large-scale

489 MDE isolations in recent years $(12,37,38,44)$. However, due to the mechanical stress of ultra-

490 high speeds and increased handling that is associated with each step, UC can suffer from

491 exosome loss (54). We also found that the UC method resulted in a large loss of MDEs in bovine

492 milk (Fig. 4). In particular, the UC-supernatant (the designated negative control) contained a

493 similar concentration of MDEs compared to the enriched pellet, indicative of an unsuccessful 
494 fractionation and a large loss of MDEs. Specific g-force / $\mathrm{k}$ factor usage and centrifugation run

495 times during UC may have contributed to this, as speeds of $100,000 \mathrm{x} \mathrm{g}$ or more can greatly

496 influence the sedimentation ratio of pure exosomes (55). Serial UC methods may need to be

497 coupled with another purification protocol, including density gradient centrifugation and/or

498 buoyant density gradient centrifugation to isolate high quality MDEs from bovine milk whey

$499(12,26,33,37,50,56)$. Nevertheless, the EQ method successfully fractionated MDEs in bovine

500 milk, where a significant difference in exosome concentration was observed between the pellet

501 and the supernatant. We found that EQ precipitation is more effective at isolating MDEs from

502 bovine milk whey compared to the UC method. However, minimal differences were seen in the

503 total RNA (Fig. 5) and soluble protein (Fig. 6) obtained between the two methods. It is therefore

504 imperative to consider the downstream molecular applications that are associated with the MDE

505 isolations prior to choosing a method. Notably, this is also one of the major recommendations of

506 ISEV2014 (28).

\section{Human Milk-derived Exosome Isolation: UC versus EQ Methods}

508 In human milk, both the UC and EQ methods successfully isolated MDEs, as evidenced by

509 the higher concentrations of exosomes (Fig. 7-8), RNA (Fig. 9), and protein (Fig. 10) in the

510 pellet fractions compared to their respective supernatants. However, similar to bovine milk, we

511 found the EQ method to be more efficient at isolating high quality intact MDEs of the correct

512 size from human milk compared to the UC method. Indeed, EQ has been shown to be far

513 superior than size exclusion, immunoaffinity, and UC methods by previous studies, as the

514 polymer-based technology alters the solubility and dispersibility of the biological fluid and force

515 insoluble EVs out of the solution $(32,33,42,57)$. Similarly, several maternal milk-based studies to

516 date have used EQ method to successfully isolate MDEs across bovine, human, and rodent milk 
$517(13,38,56,58)$. It should be noted that EQ precipitation requires pre-clean up steps and may also

518 co-precipitate non-exosome contaminants, including protein aggregates and other unwanted MVs

519 (33,54). As such, in order to accurately compare UC and EQ precipitation methods, in terms of

520 exosome yield, purity, and size exclusivity, we added several thorough pre-purification steps to

521 remove cellular debris, fat, cream, and casein proteins, and serial filtration steps $(0.45 \mu \mathrm{M}$ and

$5220.22 \mu \mathrm{M})$ to both methods.

\section{RNA Extraction of Bovine and Human Milk-derived Exosomes}

$524 \quad$ High RNA yield, purity, and integrity are essential parameters that can determine the validity

525 of MDE-based molecular applications, including RT-qPCR, microarrays, RNA/miRNA

526 sequencing. We found that the TRIzol LS protocol is best suited for extracting RNA from bovine

527 MDEs. Similarly, previous studies have also isolated high quality RNA, suitable for RNA

528 sequencing, from bovine MDEs using the Trizol LS protocol (47,59). Moreover, we did not

529 detect significant differences in human MDE RNA yield and purity across TRIzol LS, TRIzol +

530 RCC, and TRIzol LS + RCC protocols. We concluded that all three methods can be used to

531 isolate sequencing-quality exosome RNA from human MDEs. The QIAzol + miRNeasy Mini Kit

532 is the least preferable RNA extraction method, as this protocol produced the lowest RNA yield.

533 Although several previous studies have reported successful RNA extractions using QIAzol +

534 miRNeasy Mini Kit in bovine, mice, and human milk samples, their sample processing, exosome

535 extraction techniques, starting material, and parturient histories varied from ours

$536(11,15,21,45,48)$. For example, Oh et al. (2015) stored bovine colostrum immediately after

537 collection at $-80{ }^{\circ} \mathrm{C}$ until further use and only conducted differential centrifugations to remove

538 fat and casein proteins. Whereas, Izumi et al. (2012) stored bovine colostrum immediately at -20

$539{ }^{\circ} \mathrm{C}$ after collection and conducted serial filtrations to eliminate residual cell debris along with 
540 removing fat and casein proteins via differential centrifugation. Our samples were not stored at -

$54180{ }^{\circ} \mathrm{C}$ and/or $-20{ }^{\circ} \mathrm{C}$ immediately upon collection, and we performed serial filtration and

542 processing steps prior to MDE isolations via the UC method.

543 In conclusion, our findings indicate that it is not necessary to pre-process unpasteurized

544 bovine milk to remove cream, fat globules, and casein proteins soon after collection and prior to

545 long-term storage at $-80{ }^{\circ} \mathrm{C}$. Similar quantities and quality of MDEs can be obtained from frozen

546 whole milk, as long as the processing to remove unwanted materials is conducted prior to MDE

547 fraction. Furthermore, we have refined an EQ method, coupled with serial centrifugation and

548 filtration steps, that can be used to successfully precipitate high quality MDEs from both bovine

549 and human frozen whole milk. We also identified TRIzol LS as best suited for the isolation of

550 bovine MDE RNA, whereas TRIzol LS, TRIzol + RCC, and TRIzol LS + RCC methods can be

551 used interchangeably in human samples. Taken together, our findings provide new experimental

552 insights into MDE processing and storage requirements, fractionation techniques, RNA, and

553 protein extraction methods that can be used to establish standardized guidelines and protocols for

554 MDE isolation, characterization, and analysis. Standardized protocols will enhance the

555 comparability and reproducibility of MDE research and minimize confounding variables that

556 may mask relevant biological differences across milk samples. 


\section{Acknowledgements}

558 We like to thank Loa-De-Mede Holsteins Farm in Oshawa, Ontario, Canada for providing

559 unpasteurized, fresh, bovine milk. We like to thank Ms. Durga Archarya and Mr. Bruno Chue at

560 the Center for the Neurobiology of Stress, Toronto, Ontario, Canada for their valuable assistance

561 and guidance with Transmission Electron Microscopy. We like to thank Mr. Greg Wesney and

562 Mr. James Jorgensen at The Structural \& Biophysical Core Facility at the Peter Gilgan Center for

563 Research and Learning, Toronto, Ontario, Canada for assistance with Nanoparticle Tracking

564 Analysis. We like to also thank Dr. Deborah O'Connor and Dr. Agostino Pierro at the Hospital

565 for Sick Children for their advice and scientific guidance.

\section{Declaration of Interest Statement}

567 The authors declare that they have no competing or financial interests.

\section{$568 \quad$ Funding}

569 This work was supported by Discovery grants from the Natural Sciences and Engineering

570 Council of Canada (NSERC) to Dr. Patrick O. McGowan and Dr. Alison S. Fleming. Dr. Sanoji

571 Wijenayake holds an NSERC Postdoctoral Research Fellowship.

\section{Author Contributions}

573 SW designed the experiments. SW and SE conducted all the molecular work and analyzed the

574 data. ZT assisted in exosome fractionation experiments. MAP provided the human milk samples.

575 MAS provided the bovine milk samples. ASF provided material support. POM provided

576 necessary equipment and resources and supervised the research. SW and SE wrote the first draft

577 of the paper, and all authors contributed to revising the manuscript.

\section{Availability of data and materials}

579 The data used in this study are available from the corresponding author upon request. 


\section{$580 \quad$ References}

581 1. Ballard O, Morrow A. Human Milk Composition: Nutrients and Bioactive Factors. Pediatr $582 \quad$ Clin North Am. 2013;60:49-74.

583 2. Andreas N, Kampmann B, Mehring Le-Doare K. Human breast milk: A review on its $584 \quad$ composition and bioactivity. Early Hum Dev. 2015;91:629-35.

585 3. Chen Y, Wang J, Yang S, Utturkar S, Crodian J, Cummings S, et al. Effect of high-fat diet 586 on secreted milk transcriptome in midlactation mice. Physiol Genomics.

$587 \quad 2017 ; 49(12): 747-62$.

588 4. De Leoz M, Kalanetra K, Bokulich N, Strum J, Underwood M, German J, et al. Human 589 milk glycomics and gut microbial genomics in infant feces show a correlation between 590 human milk oligosaccharides and gut microbiota: A proof-of-concept study. J Proteome $591 \quad$ Res. 2015;14:491-502.

592 5. Hunt K, Foster J, Forney L, Schütte U, Beck D, Abdo Z, et al. Characterization of the 593 Diversity and Temporal Stability of Bacterial Communities in Human Milk. PLoS One. 2011;6:e21313.

595 6. Der G, Batty G, Deary I. Effect of breast feeding on intelligence in children: Prospective 596 study, sibling pairs analysis, and meta-analysis. Br Med J. 2006;333:945-8.

597 7. Hård A, Nilsson A, Lund A, Hansen-Pupp I, Smith L, Hellström A. Review shows that 598 donor milk does not promote the growth and development of preterm infants as well as 599 maternal milk. Acta Paediatr Int J Paediatr. 2019;108:998-1007.

600 8. Kulski JK, Hartmann PE. Changes in human milk composition during the initiation of $601 \quad$ lactation. Aust J Exp Biol Med Sci. 1981;59:101-14.

602 9. Hennet T, Borsig L. Breastfed at Tiffany's. Vol. 41, Trends in Biochemical Sciences. 
603 2016. p. 508-18.

604 10. Lässer C, Seyed Alikhani V, Ekström K, Eldh M, Torregrosa Paredes P, Bossios A, et al.

605 Human saliva, plasma and breast milk exosomes contain RNA: Uptake by macrophages. J

$606 \quad$ Transl Med. 2011;9:9.

607 11. Izumi H, Kosaka N, Shimizu T, Sekine K, Ochiya T, Takase M. Bovine milk contains

608 microRNA and messenger RNA that are stable under degradative conditions. J Dairy Sci.

$609 \quad 2012 ; 95: 4831-41$.

610 12. Miyake H, Lee C, Chusilp S, Bhalla M, Li B, Pitino M, et al. Human breast milk

611 exosomes attenuate intestinal damage. Pediatr Surg Int. 2020;36:155-63.

612 13. Hock A, Miyake H, Li B, Lee C, Ermini L, Koike Y, et al. Breast milk-derived exosomes

613 promote intestinal epithelial cell growth. J Pediatr Surg. 2017;52:755-9.

614 14. Manca S, Upadhyaya B, Mutai E, Desaulniers A, Cederberg R, White B, et al. Milk

615 exosomes are bioavailable and distinct microRNA cargos have unique tissue distribution

$616 \quad$ patterns. Sci Rep. 2018;8:1-11.

617 15. Title A, Denzler R, Stoffel M. Uptake and function studies of maternal milk-derived

618 MicroRNAs. J Biol Chem. 2015;290:23680-91.

619 16. Zempleni J, Sukreet S, Zhou F, Wu D, Mutai E. Milk-Derived Exosomes and Metabolic

620 Regulation. Annu Rev Anim Biosci. 2019;7:245-62.

621 17. Melnik B, Kakulas F, Geddes D, Hartmann P, John S, Carrera-Bastos P, et al. Milk

622 miRNAs: Simple nutrients or systemic functional regulators? Nutr Metab. 2016;13:42.

623 18. Izumi H, Kosaka N, Shimizu T, Sekine K, Ochiya T, Takase M. Time-Dependent

624 Expression Profiles of microRNAs and mRNAs in Rat Milk Whey. PLoS One.

$625 \quad 2014 ; 9: \mathrm{e} 88843$. 
626 19. Modepalli V, Kumar A, Hinds LA, Sharp JA, Nicholas KR, Lefevre C. Differential 627 temporal expression of milk miRNA during the lactation cycle of the marsupial tammar 628 wallaby (Macropus eugenii). BMC Genomics. 2014;15:1012.

629 20. Melnik B, Schmitz G. Milk's Role as an Epigenetic Regulator in Health and Disease. $630 \quad$ Diseases. 2017 Mar 15;5(1):12.

631 21. Alsaweed M, Lai C, Hartmann P, Geddes D, Kakulas F. Human milk miRNAs primarily 632 originate from the mammary gland resulting in unique miRNA profiles of fractionated 633 milk. Sci Rep. 2016;6:1-13.

634 22. Zempleni J, Aguilar-Lozano A, Sadri M, Sukreet S, Manca S, Wu D, et al. Biological 635 Activities of Extracellular Vesicles and Their Cargos from Bovine and Human Milk in $636 \quad$ Humans and Implications for Infants. J Nutr. 2017;147:3-10.

637 23. Alsaweed M, Hepworth A, Lefèvre C, Hartmann P, Geddes D, Hassiotou F. Human Milk 638 MicroRNA and Total RNA Differ Depending on Milk Fractionation. J Cell Biochem. $639 \quad 2015 ; 116: 2397-407$.

640 24. Chen CC, Liu L, Ma F, Wong CW, Guo XE, Chacko J V, et al. Elucidation of exosome 641 migration across the blood-brain barrier model in vitro. Cell Mol Bioeng [Internet]. 2016 642 Dec;9(4):509-29. Available from: http://www.ncbi.nlm.nih.gov/pubmed/28392840

643 25. EL Andaloussi S, Lakhal S, Mäger I, Wood M. Exosomes for targeted siRNA delivery 644 across biological barriers. Vol. 65, Advanced Drug Delivery Reviews. 2013. p. 391-7.

645 26. Martins T, Catita J, Rosa I, Da Cruz e Silva O, Henriques A. Exosome isolation from 646 distinct biofluids using precipitation and column-based approaches. PLoS One. 647 2018;13(6):e0198820.

648 27. Alvarez-Erviti L, Seow Y, Yin H, Betts C, Lakhal S, Wood M. Delivery of siRNA to the 
5.

651 28. Lötvall J, Hill AF, Hochberg F, Buzás EI, Vizio D Di, Gardiner C, et al. Minimal

652 experimental requirements for definition of extracellular vesicles and their functions: A

653 position statement from the International Society for Extracellular Vesicles. J Extracell

$654 \quad$ Vesicles. 2014;3(1).

655 29. Zonneveld M, Brisson A, van Herwijnen M, Tan S, van de Lest C, Redegeld F, et al.

656 Recovery of extracellular vesicles from human breast milk is influenced by sample

657 collection and vesicle isolation procedures. J Extracell Vesicles. 2014;3:24215.

658 30. García-Lara N, Escuder-Vieco D, García-Algar O, De La Cruz J, Lora D, Pallás-Alonso

659 C. Effect of freezing time on macronutrients and energy content of breastmilk. Breastfeed

$660 \quad$ Med. 2012;7(4):295-301.

661 31. Leiferman A, Shu J, Upadhyaya B, Cui J, Zempleni J. Storage of Extracellular Vesicles in 662 Human Milk, and MicroRNA Profiles in Human Milk Exosomes and Infant Formulas. J

663 Pediatr Gastroenterol Nutr. 2019;69(2):235-8.

664 32. Momen-Heravi F, Balaj L, Alian S, Mantel P, Halleck A, Trachtenberg A, et al. Current 665 methods for the isolation of extracellular vesicles. Biol Chem. 2013;394(10):1253-62.

666 33. Li P, Kaslan M, Lee S, Yao J, Gao Z. Progress in exosome isolation techniques.

667 Theranostics. 2017;7(3):789-804.

668 34. Greening D, Xu R, Ji H, Tauro B, Simpson R. Proteomic Profiling. Methods and

669 Protocols. In: Posch A, editor. Methods in Molecular Biology. 1295th ed. Humana Press

$670 \quad$ Inc.; 2015. p. 179-209.

671 35. Théry C, Amigorena S, Raposo G, Clayton A. Isolation and Characterization of Exosomes 
672

673

674 36. Lässer C. Identification and analysis of circulating exosomal microRNA in human body

675 fluids. Vol. 1024, Methods in Molecular Biology. 2013. 109-128 p.

676 37. Vaswani K, Koh YQ, Almughlliq F, Peiris H, Mitchell M. A method for the isolation and

678

694 44. Gao R, Zhang R, Qian T, Peng X, He W, Zheng S, et al. A comparison of exosomes enrichment of purified bovine milk exosomes. Reprod Biol. 2017;

38. Yamada T, Inoshima Y, Matsuda T, Ishiguro N. Comparison of methods for isolating exosomes from bovine milk. J Vet Med Sci. 2012;74:1523-5.

39. Tang Y, Huang Y, Zheng L, Qin S, Xu X, An T, et al. Comparison of isolation methods of exosomes and exosomal RNA from cell culture medium and serum. Int J Mol Med. 2017;40:834-44.

40. Tamkovich S, Yunusova N, Stakheeva M, Somov A, Frolova A, Kirushina N, et al. Isolation and characterization of exosomes from blood plasma of breast cancer and colorectal cancer patients. Biomeditsinskaya Khimiya. 2017;63:165-9.

41. Benmoussa A, Lee C, Laffont B, Savard P, Laugier J, Boilard E, et al. Commercial dairy cow milk microRNAs resist digestion under stimulated gastrointestinal tract conditions. J Nutr. 2016;146:2206-15.

42. Taylor D, Zacharias W, Gercel-taylor C. Exosome isolation for proteomic analyses and RNA profiling. Methods Mol Biol. 2011;728:235-46.

43. Prendergast E, De Souza Fonseca M, Dezem F, Lester J, Karlan B, Noushmehr H, et al. Optimizing exosomal RNA isolation for RNA-Seq analyses of archival sera specimens. PLoS One. 2018;13:e0196913. 
695 derived from different periods breast milk on protecting against intestinal organoid injury.

696 Pediatr Surg Int. 2019;35:1363-8.

697 45. Izumi H, Kosaka N, Shimizu T, Sekine K, Ochiya T, Takase M. Purification of RNA from 698 milk whey. Methods Mol Biol. 2013;1024:191-201.

699 46. Filipe V, Hawe A, Jiskoot W. Critical evaluation of nanoparticle tracking analysis (NTA) 700 by NanoSight for the measurement of nanoparticles and protein aggregates. Pharm Res. $701 \quad 2010 ; 27: 796-810$.

702 47. Jin X lu, Wei Z hai, Liu L, Liu H yun, Liu J xin. Comparative studies of two methods for 703 miRNA isolation from milk whey. J Zhejiang Univ Sci B. 2015 Jun 29;16(6):533-40.

704 48. Oh S, Park M, Son J, Kim Y. Comparison of Total RNA Isolation Methods for Analysis 705 of Immune-Related microRNAs in Market Milks. Korean J Food Sci An. 2015;35:459-65.

706 49. van Herwijnen M, Driedonks T, Snoek B, Kroon A, Kleinjan M, Jorritsma R, et al. 707 Abundantly Present miRNAs in Milk-Derived Extracellular Vesicles Are Conserved $708 \quad$ Between Mammals. Front Nutr. 2018;5:81.

709 50. Li R, Dudemaine P, Zhao X, Lei C, Ibeagha-Awemu E. Comparative Analysis of the 710 miRNome of Bovine Milk Fat, Whey and Cells. PLoS One. 2016;11:e0154129.

711 51. Howard KM, Jati Kusuma R, Baier SR, Friemel T, Markham L, Vanamala J, et al. Loss of 712 miRNAs during processing and storage of cow's (Bos taurus) milk. J Agric Food Chem. 2015;63(2):588-92.

714 52. Munagala, Radha, Farrukh Aqil JJ, Gupta RC. Bovine milk-derived exosomes for drug 715 delivery. Cancer Lett. 2016;371(1):48-61.

716 53. Hata T, Murakami K, Nakatani H, Yamamoto Y, Matsuda T, Aoki N. Isolation of bovine 717 milk-derived microvesicles carrying mRNAs and microRNAs. Biochem Biophys Res 
718 Commun. 2010 May 28;396(2):528-33.

719 54. Witwer KW, Buzás EI, Bemis LT, Bora A, Lässer C, Lötvall J, et al. Standardization of

720 sample collection, isolation and analysis methods in extracellular vesicle research. $\mathbf{J}$

$721 \quad$ Extracell Vesicles. 2013;2(1).

722 55. Jeppesen DK, Hvam ML, Primdahl-Bengtson B, Boysen AT, Whitehead B, Dyrskjøt L, et

723 al. Comparative analysis of discrete exosome fractions obtained by differential

724 centrifugation. J Extracell Vesicles. 2014;3(1).

725 56. Yu L-L, Zhu J, Liu J-X, Jiang F, Ni W-K, Qu L-S, et al. A Comparison of Traditional and

726 Novel Methods for the Separation of Exosomes from Human Samples. Biomed Res Int.

$2018 ; 2018$.

728 57. Alvarez ML, Khosroheidari M, Kanchi Ravi R, Distefano JK. Comparison of protein, 729 microRNA, and mRNA yields using different methods of urinary exosome isolation for 730 the discovery of kidney disease biomarkers. Kidney Int. 2012 Nov 1;82(9):1024-32.

731 58. Qin W, Tsukasaki Y, Dasgupta S, Mukhopadhyay N, Ikebe M, Sauter ER. Exosomes in

732 human breast milk promote EMT. Clin Cancer Res. 2016 Sep 1;22(17):4517-24.

733 59. Benmoussa A, Laugier J, Beauparlant CJ, Lambert M, Droit A, Provost P. Complexity of 734 the microRNA transcriptome of cow milk and milk-derived extracellular vesicles isolated 735 via differential ultracentrifugation. J Dairy Sci. 2020 Jan 1;103(1):16-29.

736

737

738 


\section{Figure Captions}

740 Figure 1. Pre-processing of whole milk prior to long-term storage of bovine milk. Group 1)

741 unprocessed whole milk, group 2) milk without fat globules and cream, and group 3) isolated

742 whey fraction without fat globules, cream and casein proteins. Exosome concentration

743 [particles/mL] isolated via ExoQuick and ultracentrifugation methods across G1-G3, as

744 determined by Nanoparticle Tracking Analysis (NTA) (A); size and distribution profiles of milk-

745 derived exosomes as determined by NTA (B); RNA concentration $[\mathrm{ng} / \mu \mathrm{L}](\mathrm{C})$; RNA purity

746 (A260/A280 and A260/A230) (D-E); and protein concentration $[\mu \mathrm{g} / \mathrm{mL}](\mathrm{F})$ obtained from the

747 isolated exosomes. Data are mean \pm SEM with $n=3$ independent trials/group. For NTA 3 video

748 frames of $30 \mathrm{~s}$ each were used. Data were analyzed using one-way analysis of variance with a

749 Tukey post-hoc test $(\mathrm{p} \leq 0.05)$ for main effect of pre-processing groups.

750

751 Figure 2. Morphology of bovine milk-derived exosomes visualized by Transmission Electron

752 Microscopy (TEM) with negative staining (uranyl acetate). Milk-derived exosomes were

753 isolated via ExoQuick precipitation method. Group 1) unprocessed whole milk, group 2) milk

754 without fat globules and cream, and group 3) isolated whey fraction without fat globules, cream

755 and casein proteins. Scale bars: $200 \mathrm{~nm}-500 \mathrm{~nm}$.

756

757 Figure 3. Morphology of bovine milk-derived exosomes visualized by Transmission Electron

758 Microscopy (TEM) with negative staining (uranyl acetate). Milk-derived exosomes were isolated

759 via ultracentrifugation method. Group 1) unprocessed whole milk, group 2) milk without fat

760 globules and cream, and group 3) isolated whey fraction without fat globules, cream and casein

761 proteins. Scale bars: $200 \mathrm{~nm}-1000 \mathrm{~nm}$. 
762 Figure 4. Size and distribution profiles of bovine milk-derived exosomes as determined by

763 Nanoparticle Tracking Analysis (NTA). Exosomes were isolated via ExoQuick precipitation and

764 ultracentrifugation methods. Exosome concentration [particles/mL] in pellets and the

765 corresponding negative controls (the supernatants) (A). Size and distribution profiles of the

766 absolute control (1x PBS) (B). Size and distribution profiles of the ExoQuick exosome pellet (C).

767 Size and distribution profiles of the ExoQuick negative control (D). Size and distribution profiles

768 of the ultracentrifugation exosome pellet (E). Size and distribution profiles of the

769 ultracentrifugation negative control $(\mathrm{F})$. Data are mean \pm SEM with $\mathrm{n}=6$ independent

770 trials/group. For NTA, 3 video frames of $30 \mathrm{~s}$ each were used. Data were analyzed using a two-

771 way analysis of variance with a Tukey post-hoc test $(\mathrm{p} \leq 0.05)$. Main effect of fractionation: * (p

$772 \leq 0.05)$. Main effect of exosome isolation method: $\$(\mathrm{p} \leq 0.05)$. Fractionation/exosome isolation

773 method interaction: $* \$(\mathrm{p} \leq 0.05)$

774

775 Figure 5. RNA yield $[\mathrm{ng} / \mu \mathrm{L}]$, purity and quality of bovine milk-derived exosome pellets and

776 supernatants isolated via ExoQuick precipitation and ultracentrifugation methods. RNA was

777 extracted using four protocols, 1) QIAzol + miRNeasy MiniKit, 2) TRIzol LS, 3) TRIzol + RNA

778 Clean and Concentrator Kit (RCC), and 4) TRIzol LS + RCC. RNA concentration [ng/ $\mu \mathrm{L}](\mathrm{A}), 1$

$779 \%$ TAE agarose gel electrophoresis (B), RNA purity - absorbance at 260nm/280nm (C), and

780 absorbance at 260nm/230nm (D). Data are mean \pm SEM with $\mathrm{n}=6$ independent trials/group.

781 Data were analyzed using a three-way analysis of variance with a Tukey post-hoc test $(\mathrm{p} \leq 0.05)$.

782 Main effect of fractionation: * $(\mathrm{p} \leq 0.05)$. Main effect of exosome isolation method: $\$(\mathrm{p} \leq 0.05)$.

783 Main effect of RNA extraction protocol: \# $(\mathrm{p} \leq 0.05)$. Fractionation/exosome isolation

784 interaction: $* \$(\mathrm{p} \leq 0.05)$. Fractionation/RNA extraction interaction: $* \#(\mathrm{p} \leq 0.05)$. Exosome 
785 isolation/RNA extraction interaction: $\$ \#(p \leq 0.05)$. $p$-values on top of supernatant bars indicate

786 significant difference between pellets and the corresponding supernatants of that particular

787 exosome isolation protocol and RNA extraction protocol.

788

789 Figure 6. Protein concentration $[\mu \mathrm{g} / \mathrm{mL}]$ of bovine milk-derived exosome pellets and 790 supernatants isolated via ExoQuick precipitation and ultracentrifugation methods. Total soluble 791 proteins were extracted from the lower organic phase resulting from four RNA extraction 792 protocols, 1) QIAzol + miRNeasy MiniKit, 2) TRIzol LS, 3) TRIzol + RNA Clean and 793 Concentrator Kit (RCC), and 4) TRIzol LS + RCC. Data are mean \pm SEM with $\mathrm{n}=6$ 794 independent trial/group. Data were analyzed using a three-way analysis of variance with a Tukey 795 post-hoc test $(\mathrm{p} \leq 0.05)$. Main effect of fractionation: $*(\mathrm{p} \leq 0.05)$. Fractionation/exosome

796 isolation interaction: $* \$(p \leq 0.05)$. Fractionation/RNA extraction interaction: $* \#(p \leq 0.05)$. $p$ 797 values on top of supernatant bars indicate significant difference between pellets and the 798 corresponding supernatants of that particular exosome isolation protocol and RNA extraction 799 protocol.

800

801 Figure 7. Morphology of human milk-derived exosomes visualized by Transmission Electron 802 Microscopy (TEM) with negative staining (uranyl acetate). Human milk-derived exosomes were 803 isolated via ExoQuick precipitation and ultracentrifugation methods. Scale bars: $200 \mathrm{~nm}$.

805 Figure 8. Size and distribution profiles of human milk-derived exosomes as determined by 806 Nanoparticle Tracking Analysis (NTA). Exosomes were isolated via ExoQuick precipitation and 807 ultracentrifugation methods. Exosome concentration [particles/mL] of the pellet and the 
808 corresponding negative controls (the supernatants) (A). Size and distribution profiles of the 809 absolute control (1x PBS) (B). Size and distribution profiles of the ExoQuick exosome pellet (C).

810 Size and distribution profiles of the ExoQuick negative control (D). Size and distribution profiles

811 of the ultracentrifugation exosome pellet (E). Size and distribution profiles of the

812 ultracentrifugation negative control (F). Data are mean \pm SEM with $\mathrm{n}=6$ independent

813 trials/group. For NTA, 3 video frames of $30 \mathrm{~s}$ each were used. Data were analyzed using a two-

814 way analysis of variance with a Tukey post-hoc test $(\mathrm{p} \leq 0.05)$. Main effect of fractionation: * (p

$815 \leq 0.05)$. Main effect of exosome isolation method: $\$(\mathrm{p} \leq 0.05)$. Fractionation/exosome isolation

816 method interaction: $* \$(p \leq 0.05)$.

818 Figure 9. RNA yield $[\mathrm{ng} / \mu \mathrm{L}]$, purity and quality of human milk-derived exosome pellets and 819 supernatants isolated via ExoQuick precipitation and ultracentrifugation methods. RNA was 820 extracted using four protocols, 1) QIAzol + miRNeasy MiniKit, 2) TRIzol LS, 3) TRIzol + RNA 821 Clean and Concentrator Kit (RCC), and 4) TRIzol LS + RCC. RNA concentration [ng/ $\mu \mathrm{L}](\mathrm{A}), 1$ $822 \%$ TAE agarose gel electrophoresis of exosome RNA (B), RNA purity - absorbance at $823260 \mathrm{~nm} / 280 \mathrm{~nm}(\mathrm{C})$, and absorbance at 260nm/230nm (D). Data are mean \pm SEM with $\mathrm{n}=3$ 824 technical replicates/exosome isolation method /RNA extraction protocol. Data were analyzed 825 using a three-way analysis of variance with a Tukey post-hoc test $(\mathrm{p} \leq 0.05)$. Main effect of 826 fractionation: * $(\mathrm{p} \leq 0.05)$. Main effect of exosome isolation method: $\$(\mathrm{p} \leq 0.05)$. Main effect of 827 RNA extraction protocol: \# $(\mathrm{p} \leq 0.05)$. Fractionation/exosome isolation interaction: $* \$(\mathrm{p} \leq$ 828 0.05). Fractionation/RNA extraction interaction: *\# $(\mathrm{p} \leq 0.05)$. Exosome isolation/RNA 829 extraction interaction: \$\# $(\mathrm{p} \leq 0.05)$. $\mathrm{p}$-values on top of supernatant bars indicate significant 
830 difference between pellets and the corresponding supernatants of that particular exosome

831 isolation protocol and RNA extraction protocol.

832 Figure 10. Protein concentration $[\mu \mathrm{g} / \mathrm{mL}]$ of human milk-derived exosome pellets and 833 supernatants isolated via ExoQuick precipitation and ultracentrifugation methods. Total soluble

834 proteins were extracted from the lower organic phase resulting from four RNA extraction 835 protocols, 1) QIAzol + miRNeasy MiniKit, 2) TRIzol LS, 3) TRIzol + RNA Clean and 836 Concentrator Kit (RCC), and 4) TRIzol LS + RCC. Data are mean \pm SEM with $\mathrm{n}=6$ 837 independent trial/group Data were analyzed using a three-way analysis of variance with a Tukey 838 post-hoc test $(\mathrm{p} \leq 0.05)$. Main effect of fractionation: $*(\mathrm{p} \leq 0.05)$. Main effect of exosome 839 isolation method: $\$(\mathrm{p} \leq 0.05)$. Main effect of RNA extraction protocol: \# $(\mathrm{p} \leq 0.05)$. $\mathrm{p}$-values on 840 top of supernatant bars indicate significant difference between pellets and the corresponding 841 supernatants of that particular exosome isolation protocol and RNA extraction protocol.

\section{Supplementary Figure Captions}

844 Supplementary Figure 1. Pre-processing of whole bovine milk prior to long-term storage at -80

$845{ }^{\circ} \mathrm{C}$. Group 1) Unprocessed whole milk stored directly at $-80{ }^{\circ} \mathrm{C}$. Group 2) Samples without 846 cream fat globules. Group 3) Whey fractions without cream, fat globules and casein proteins.

847 Supplementary Figure 2. Bovine and human milk-derived exosome isolation via enhanced 848 ExoQuick precipitation and ultracentrifugation methods.

849 Supplementary Figure 3. Optimized sample preparation protocol for Transmission Electron 850 Microscopy (TEM) for the visualization of milk-derived exosomes. Copper grids were 851 negatively stained with $2 \%$ uranyl acetate (UA). 
852 Supplementary Figure 4. Four optimized protocols, 1) QIAzol + miRNeasy MiniKit, 2) TRIzol

853 LS, 3) TRIzol + RNA Clean and Concentrator Kit (RCC), and 4) TRIzol LS + RCC used for the 854 isolation of total RNA from isolated milk exosomes. 
$A(-\infty)$

Exosome Concentration [Particles/mL]

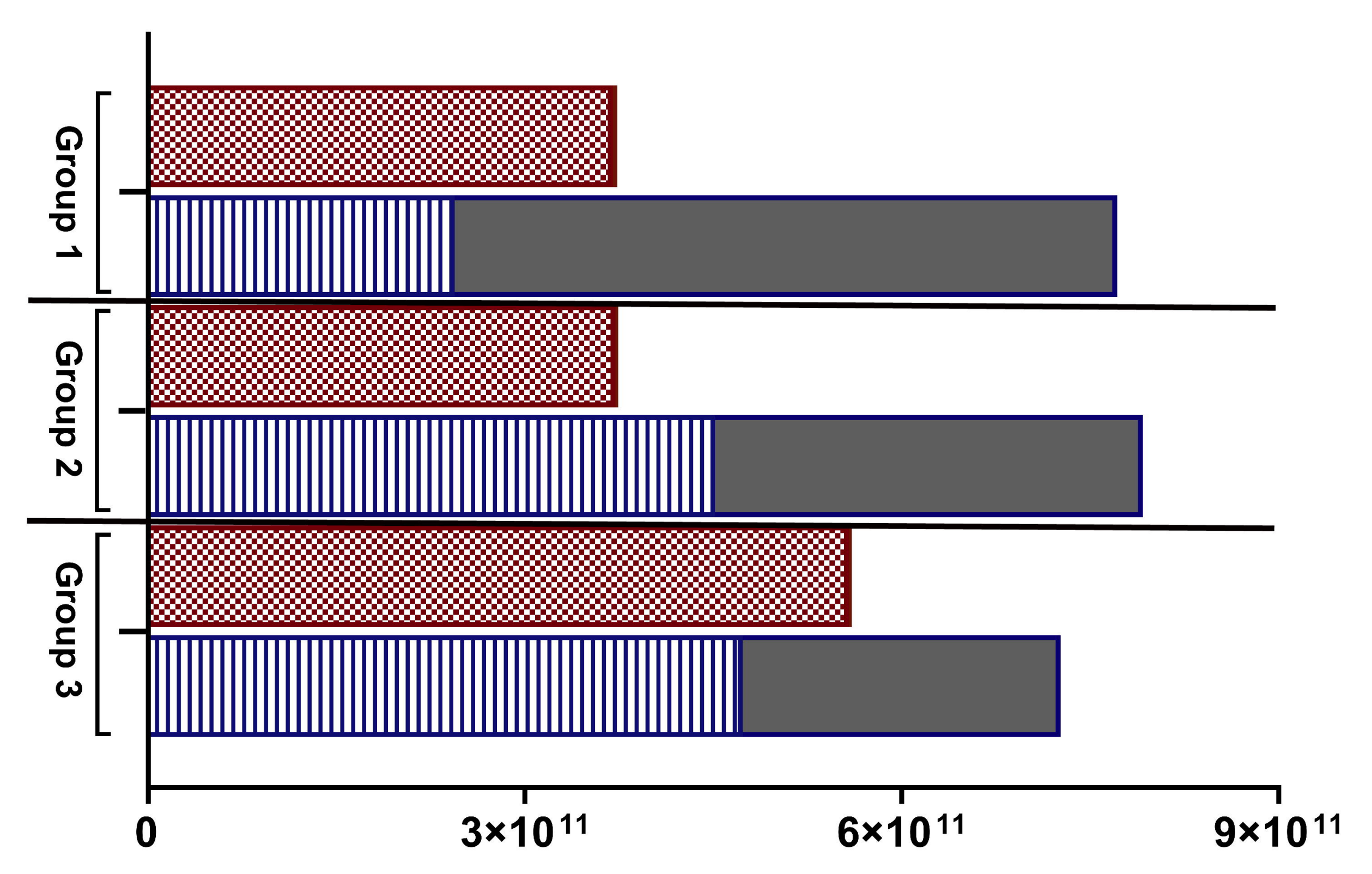

C

RNA Concentration [ng/uL]

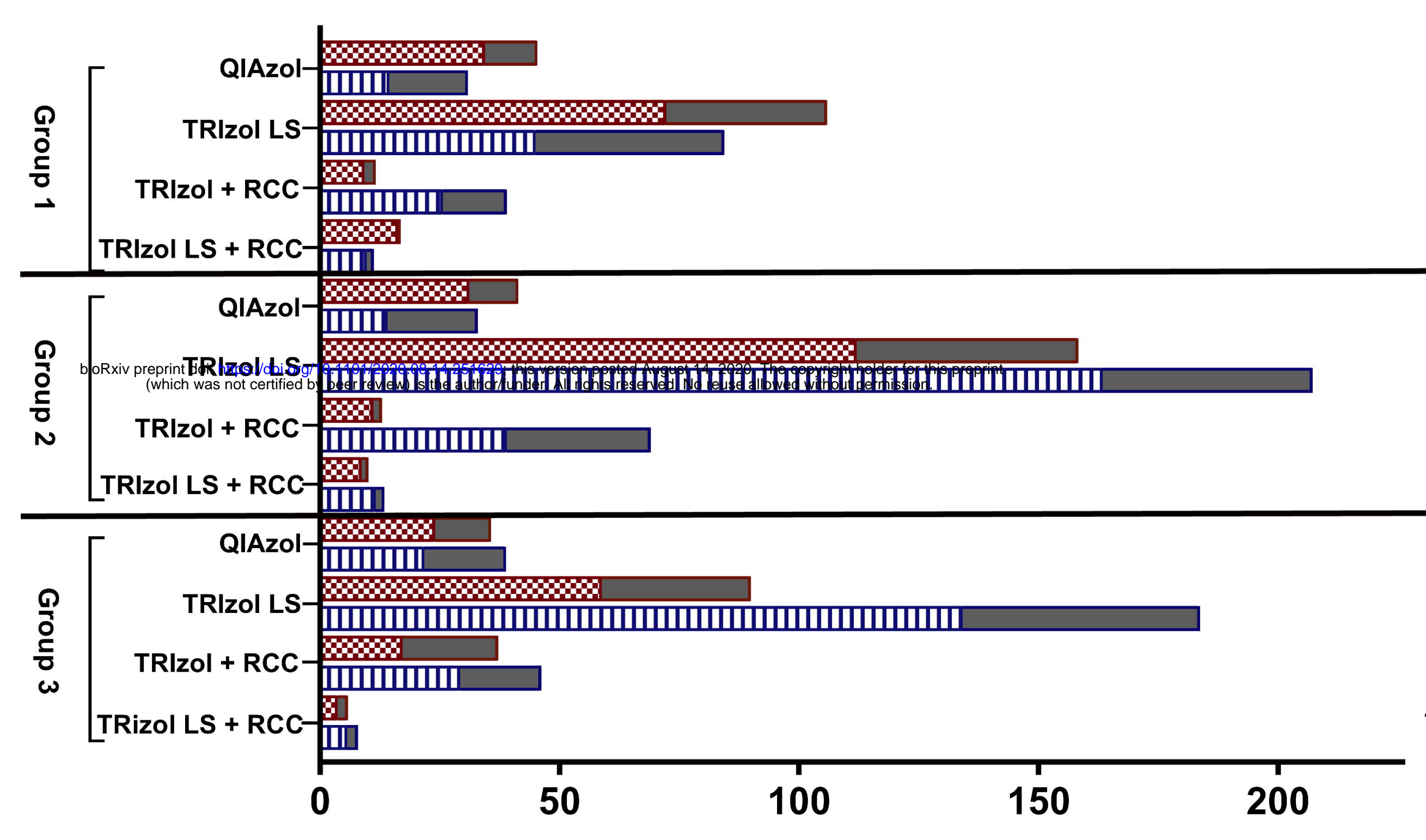

$\mathbf{F}$

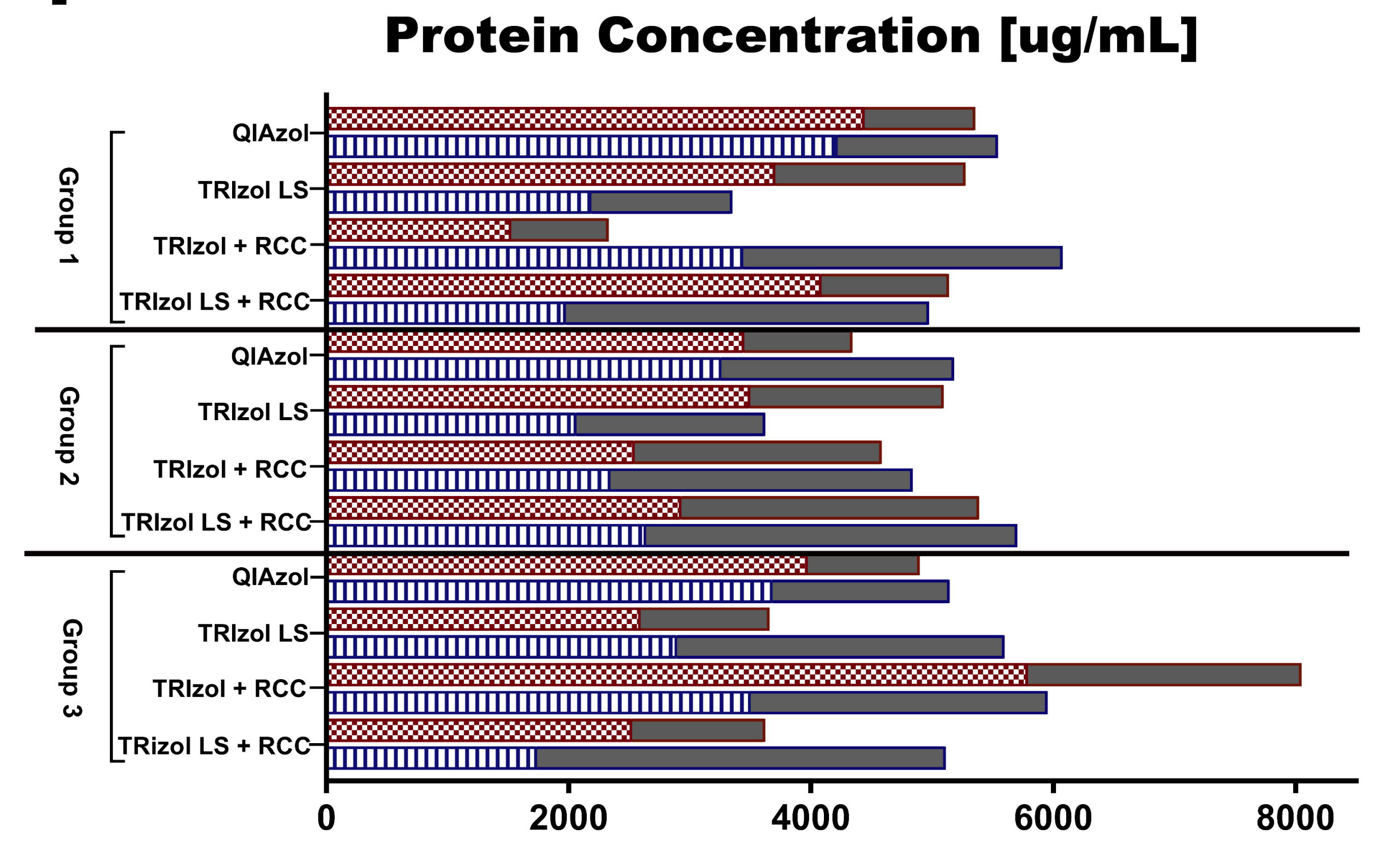

G1 / ExoQuick Pellet 1

G2 / ExoQuick Pellet 1

G3 / ExoQuick Pellet 1
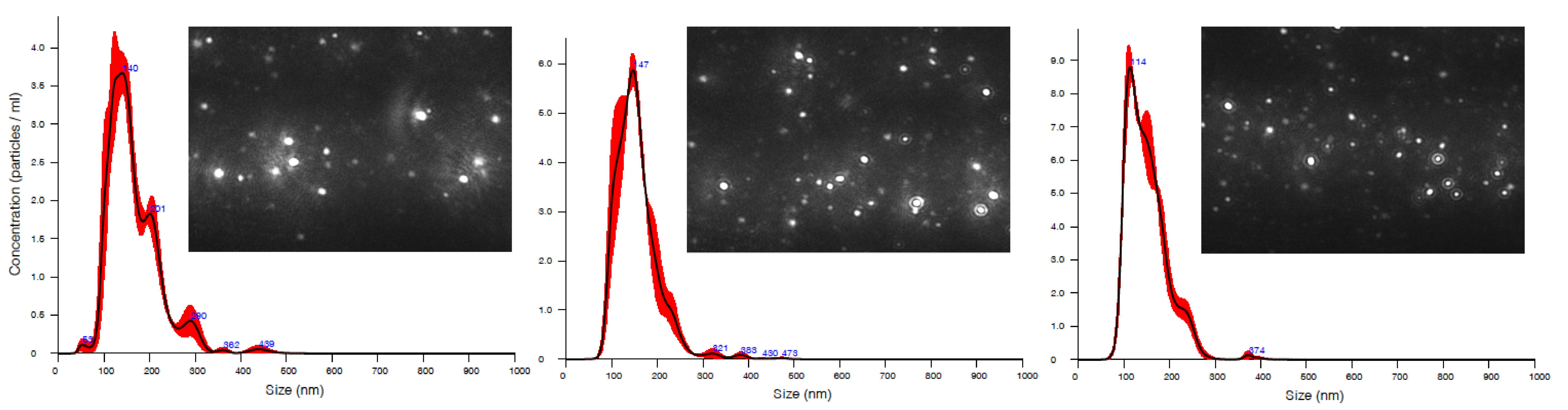

D

\section{RNA Purity A260/280}

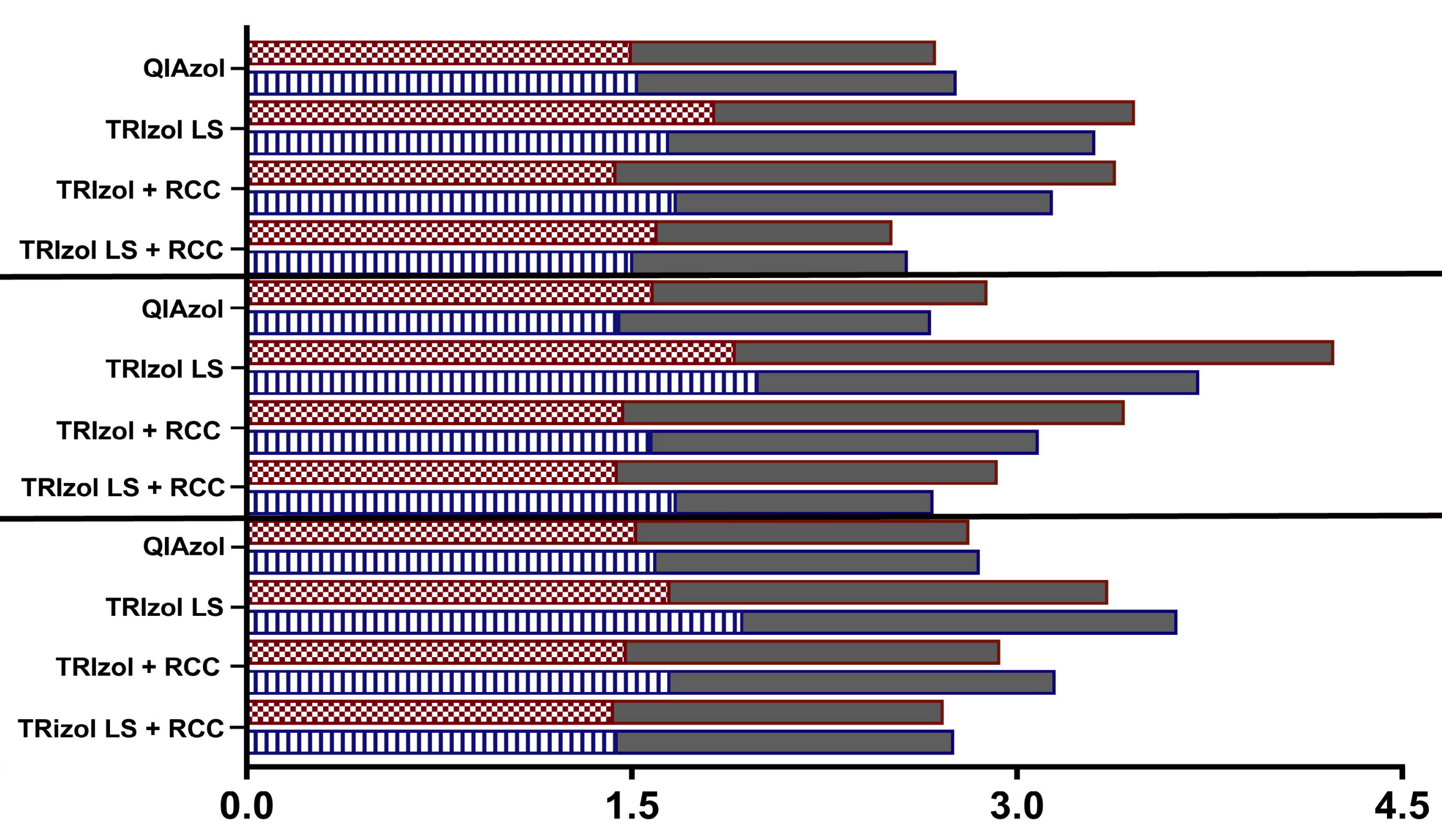

E

RNA Purity A260/230

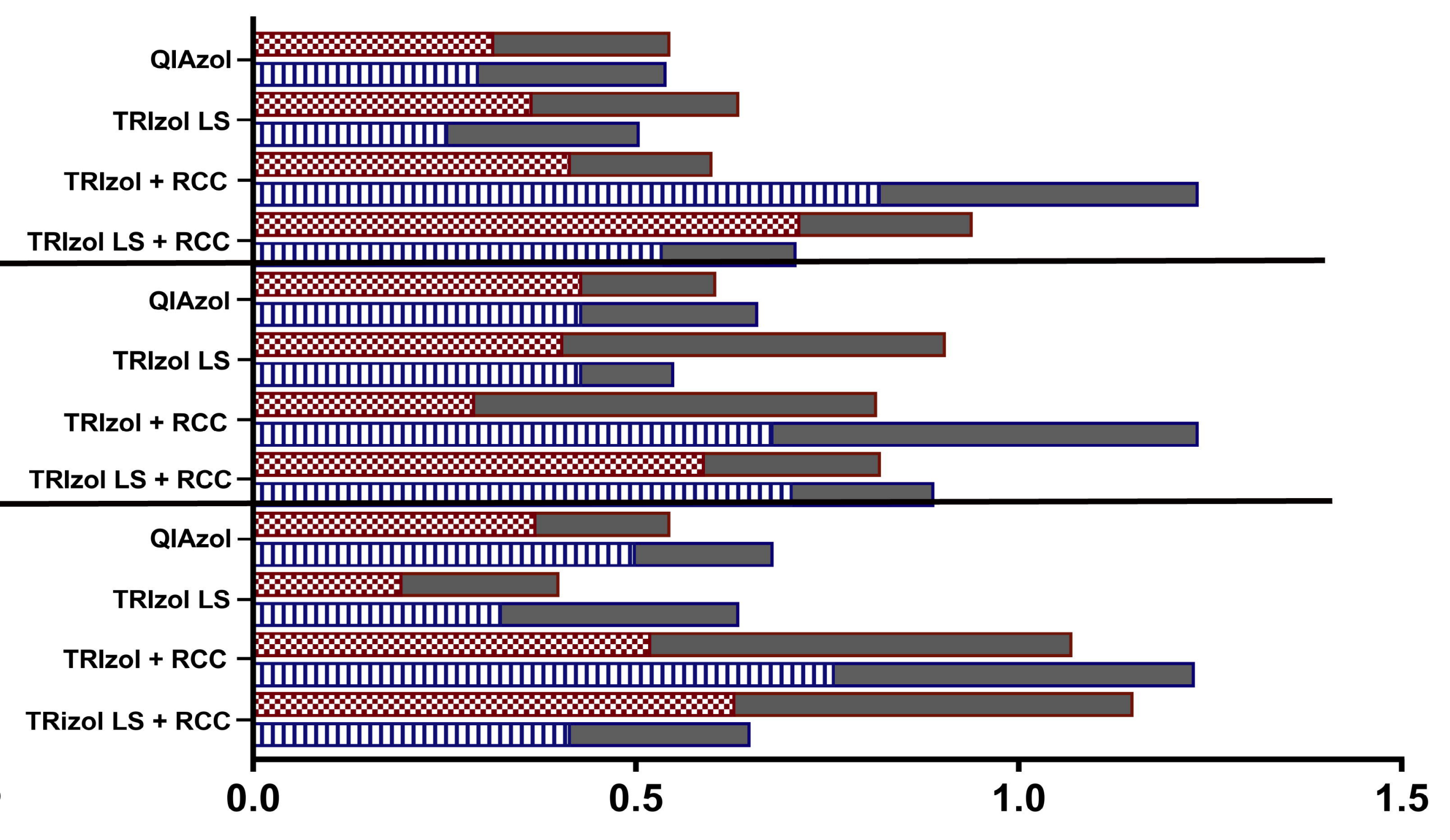

ExoQuick Pellet ||W Ultra Pellet

$\square$ ExoQuick Supernatant $\square$ Ultra Supernatant 
G1: Unprocessed

\section{Whole Milk}

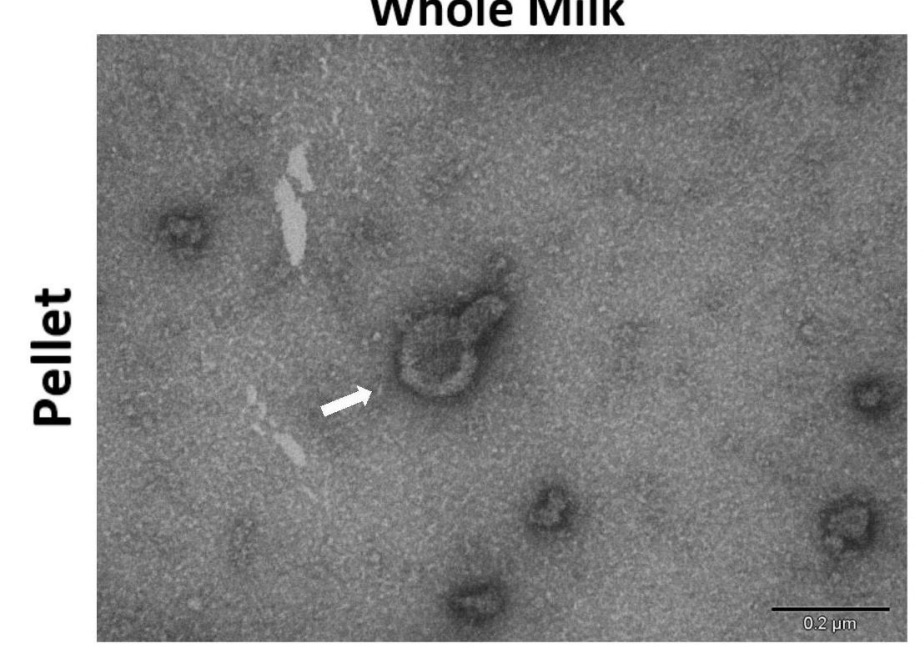

G2: w/o Cream \& Fat Globules
G3: w/o Cream, Fat Globules \& Casein Proteins

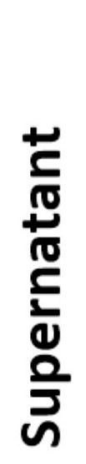


G1: Unprocessed

Whole Milk

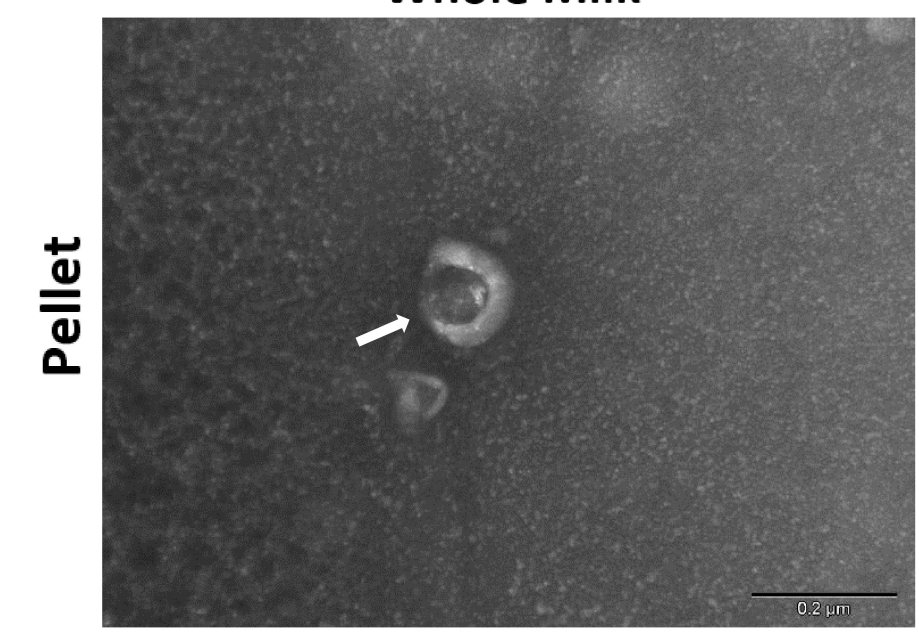



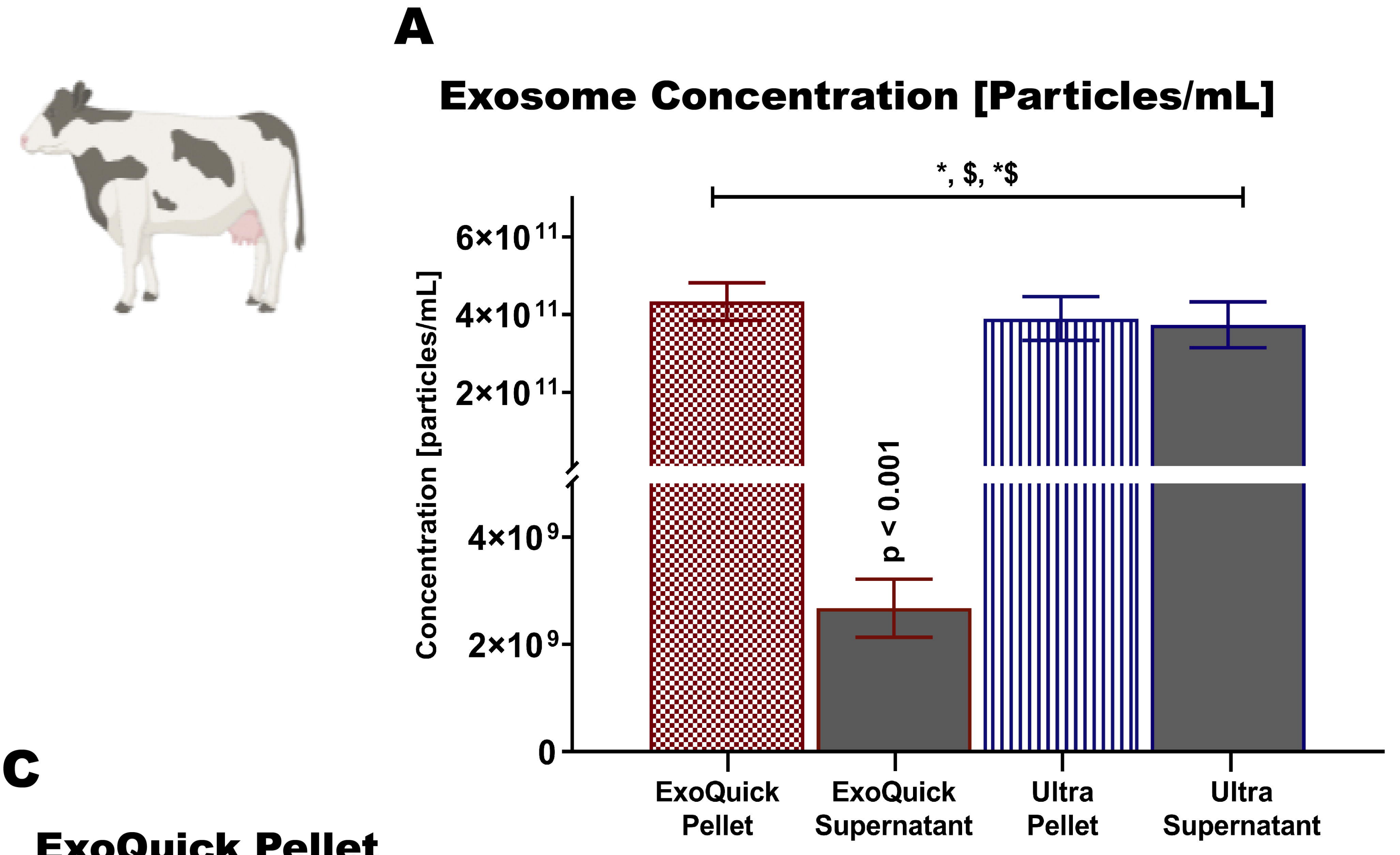

\section{ExoQuick Pellet}

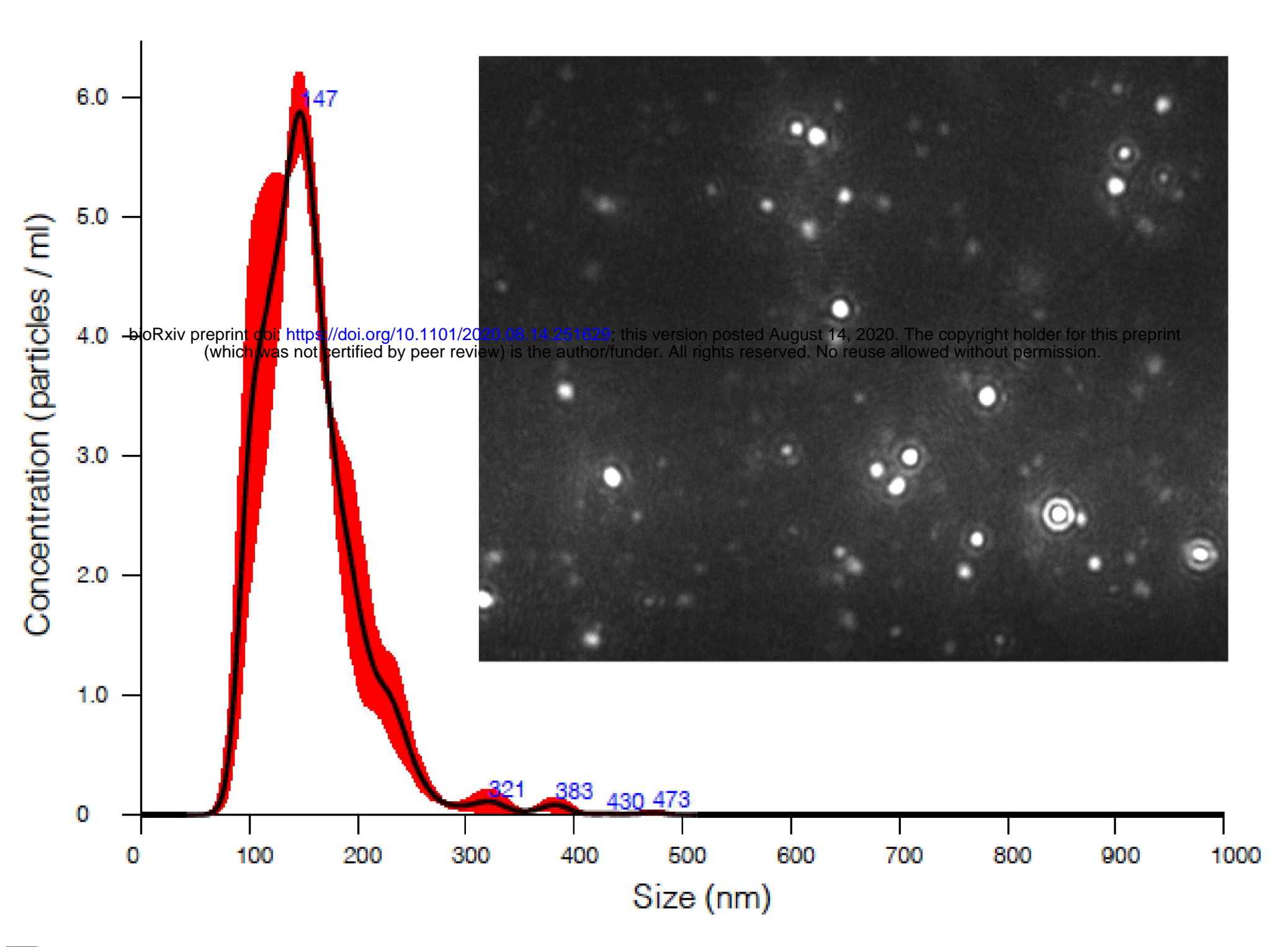

D

\section{ExoQuick Supernatant}

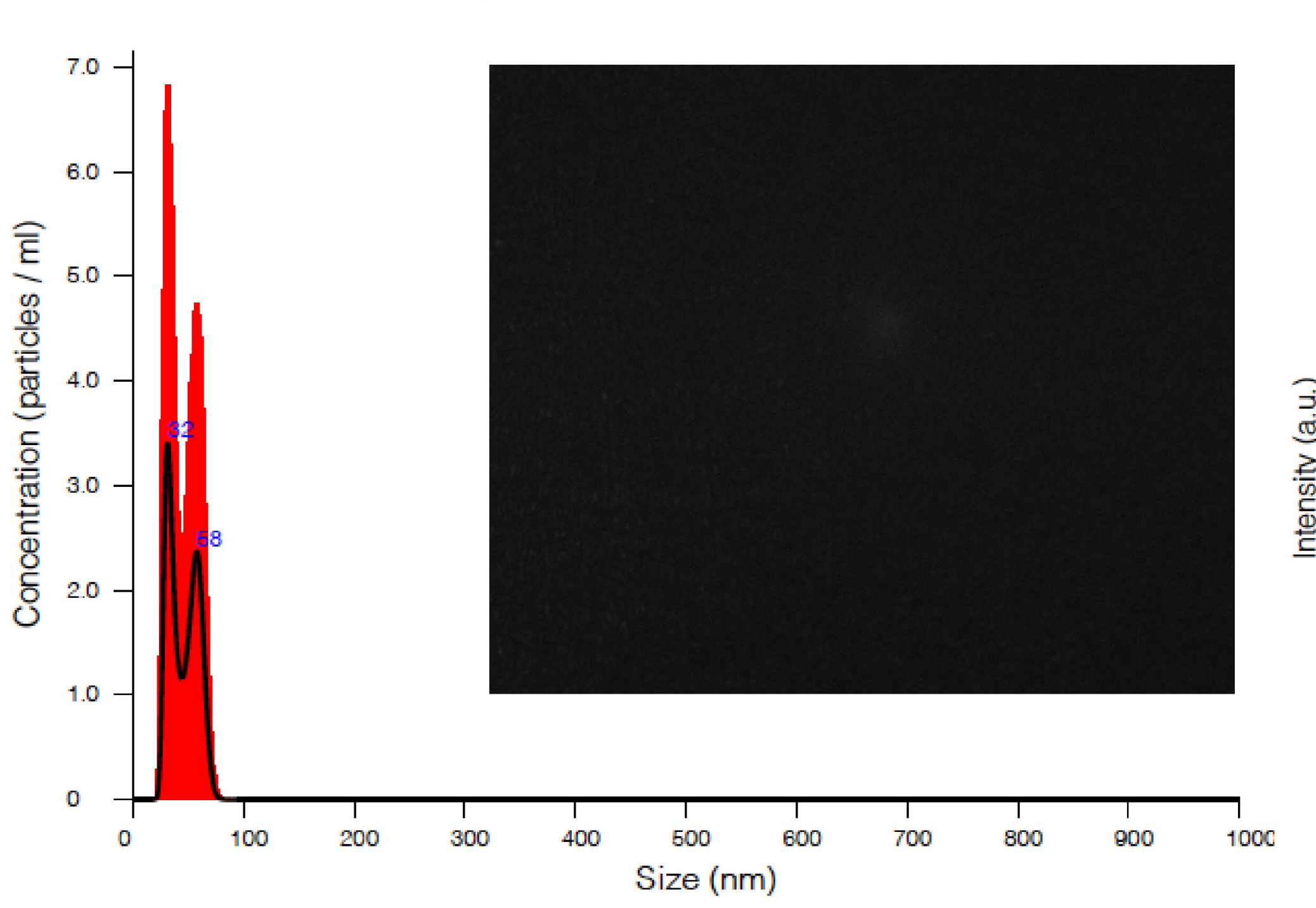

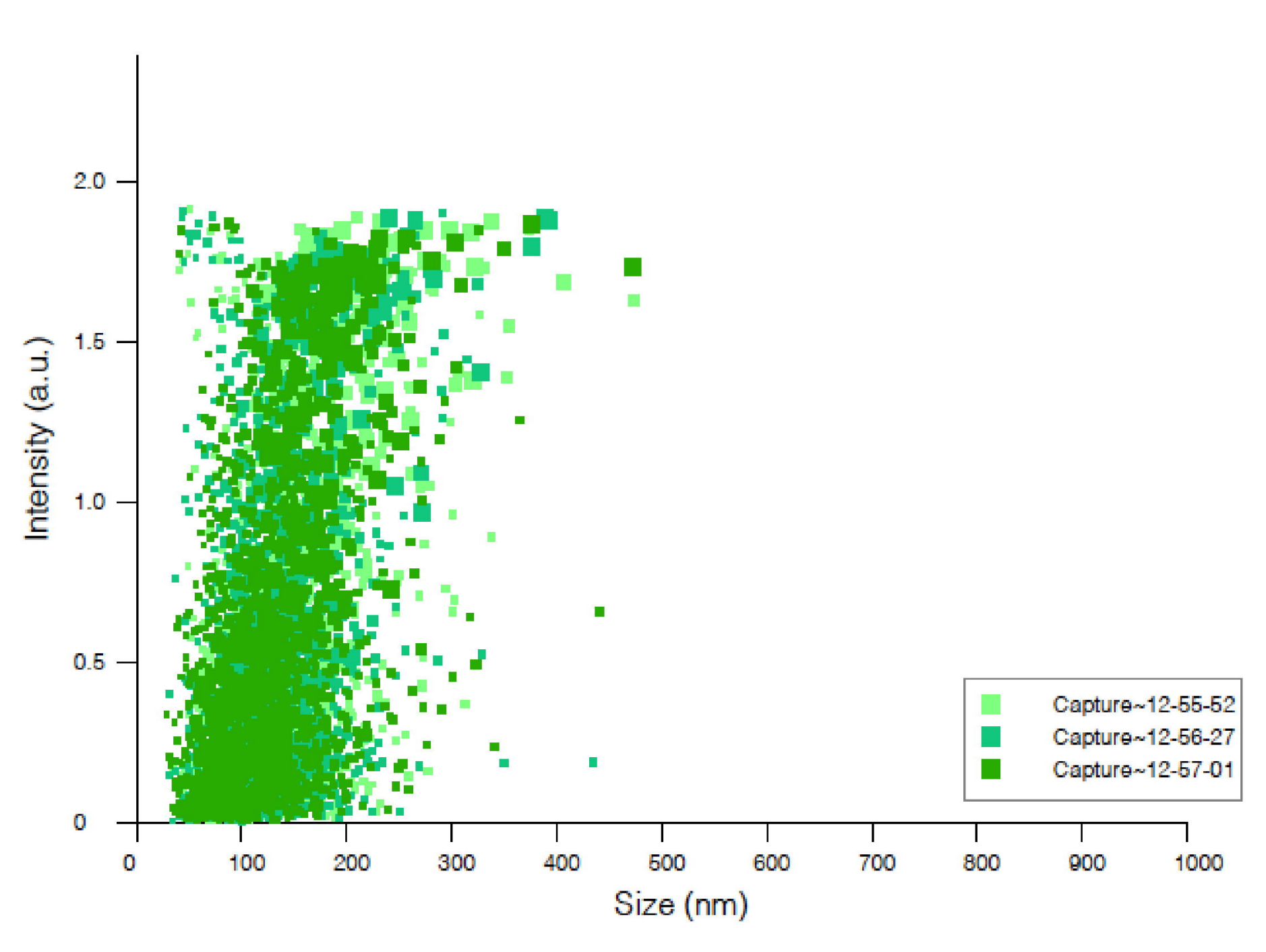

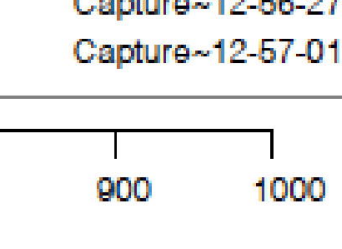

\section{$\mathbf{F}$}

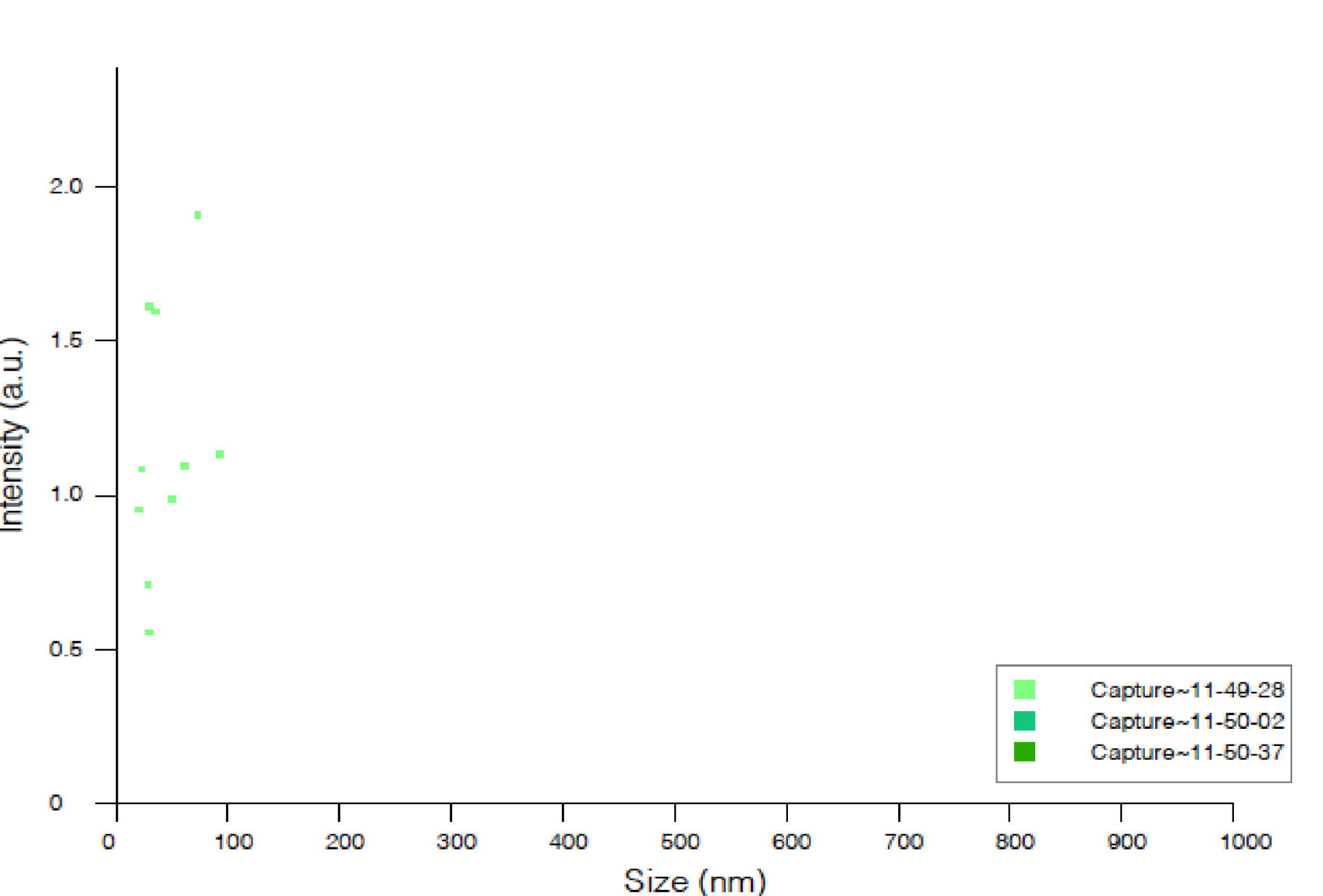

PBS Negative Control [Particles/mL]

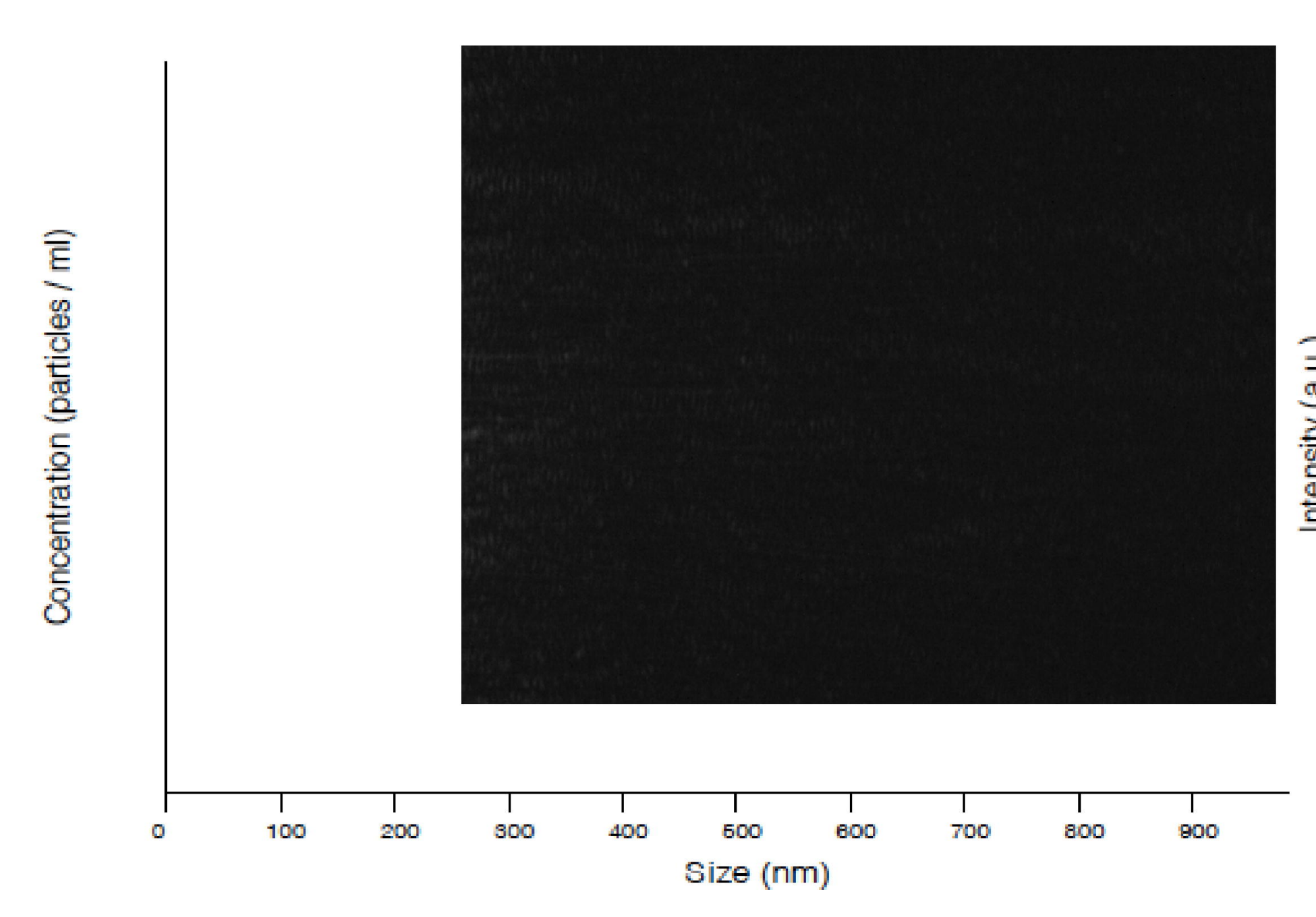

E

\section{Ultra Pellet}
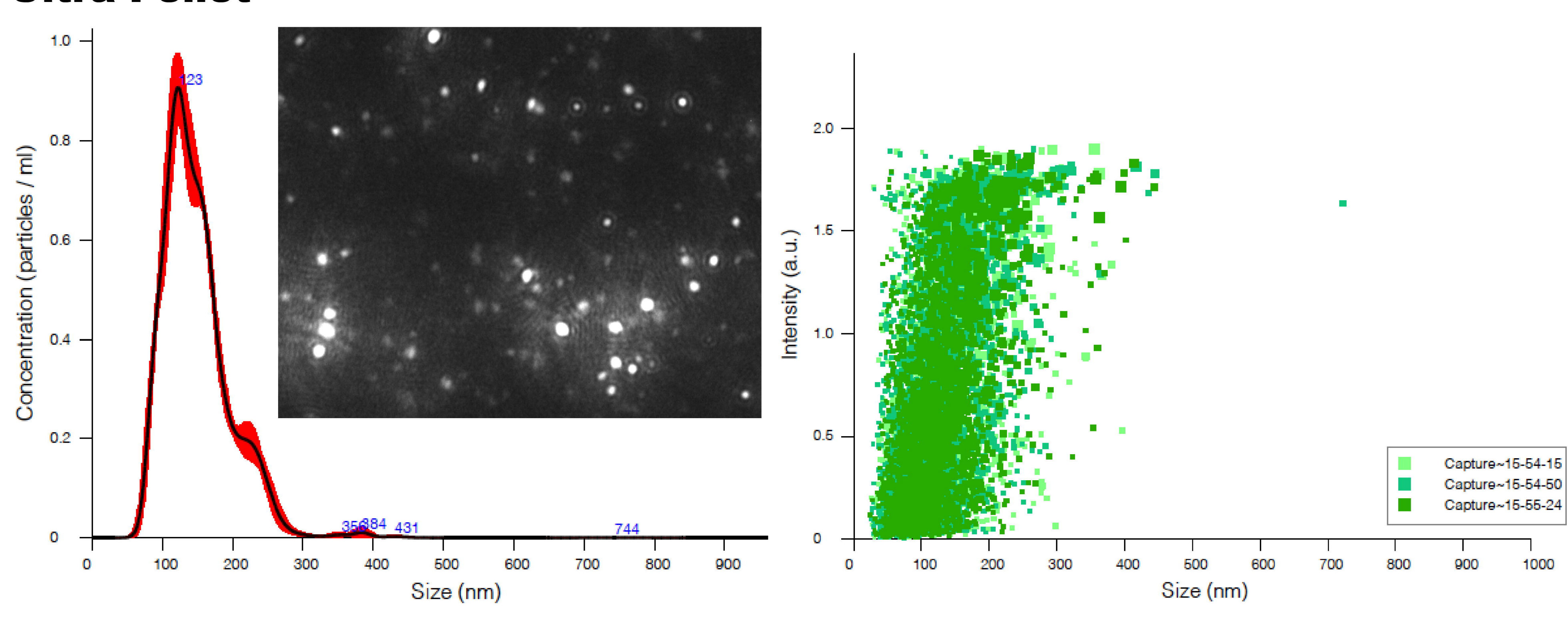

\section{Ultra Supernatant}

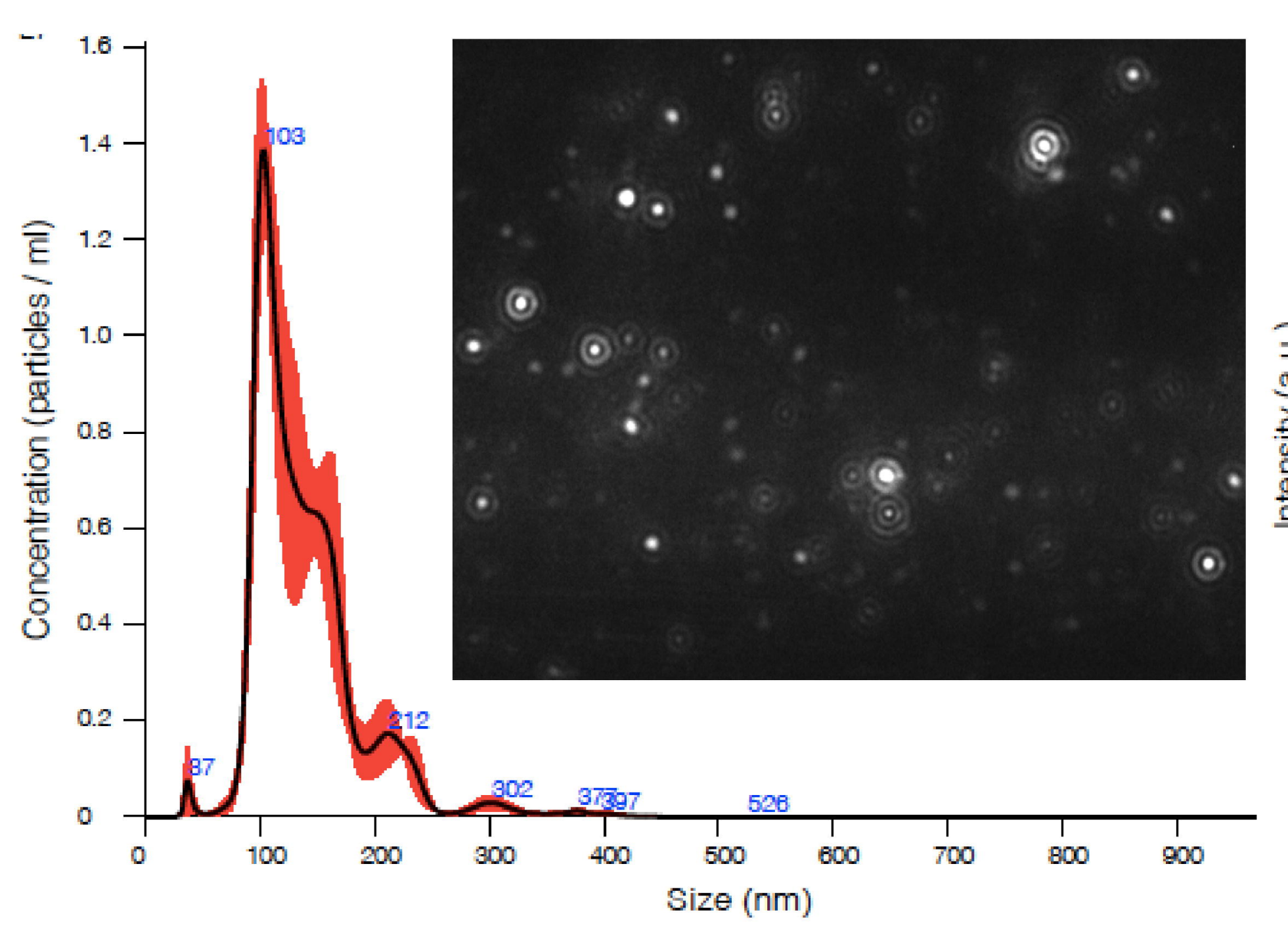

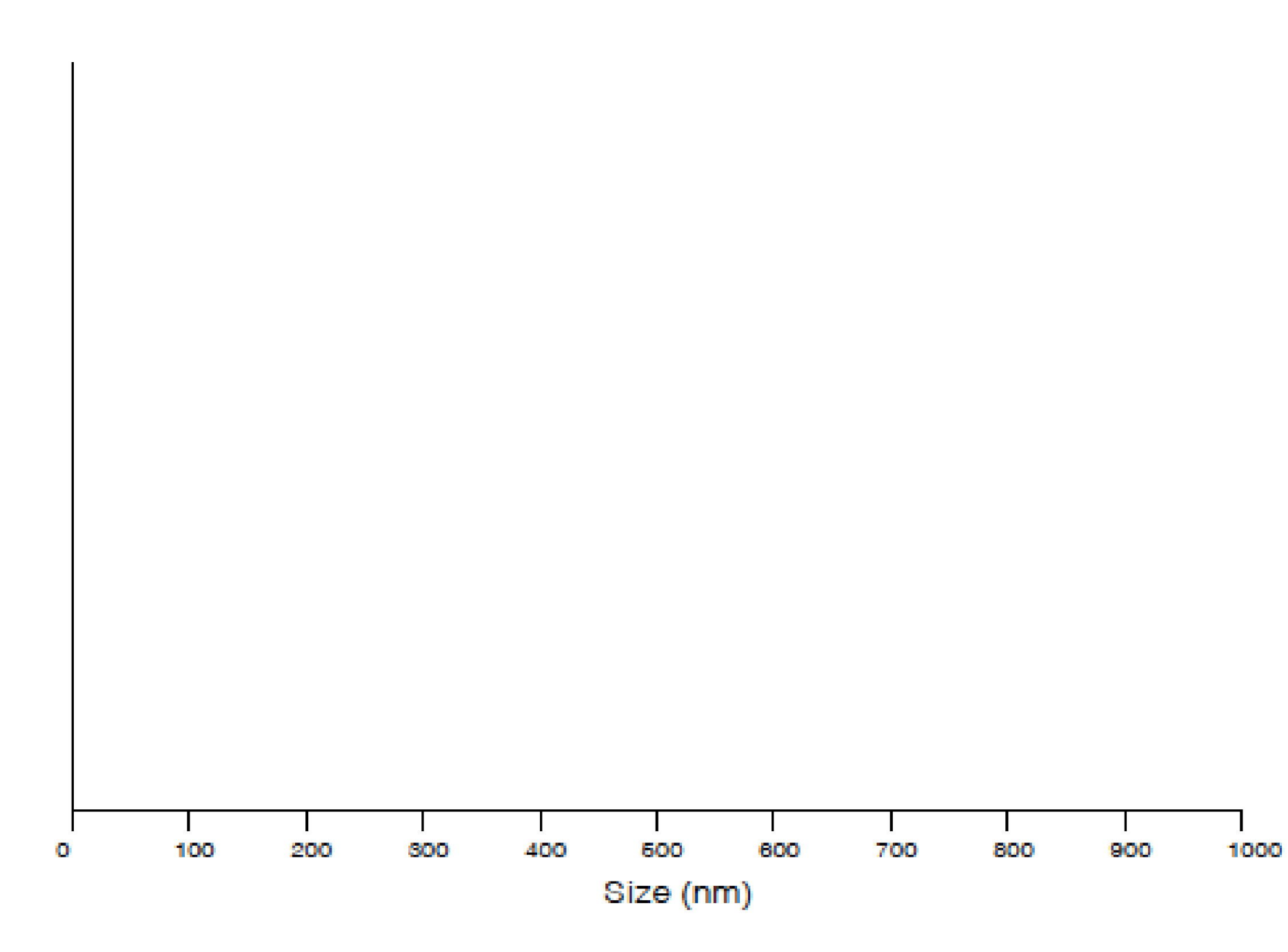

Size $(\mathrm{nm})$

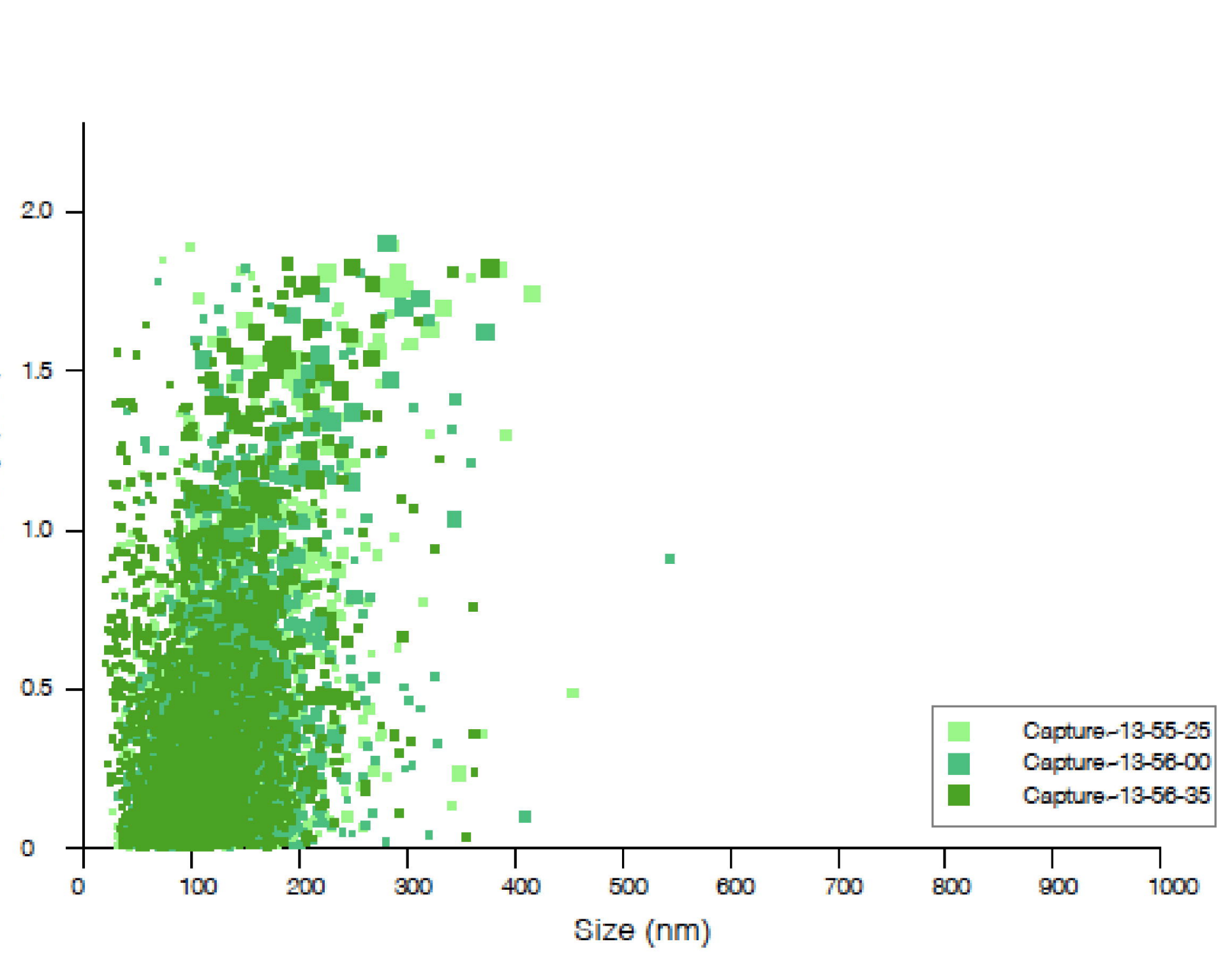


$\mathbf{A}$

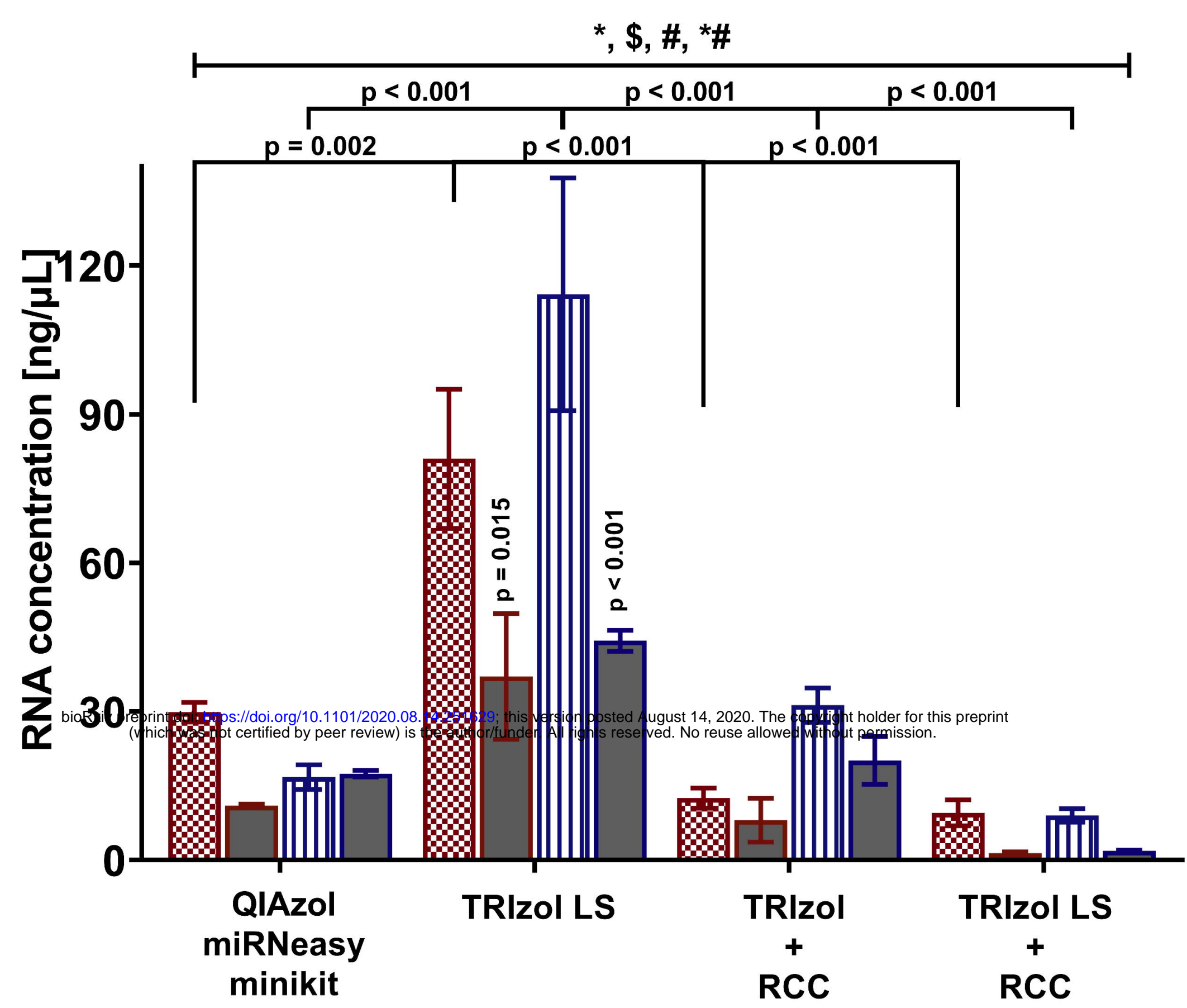

C

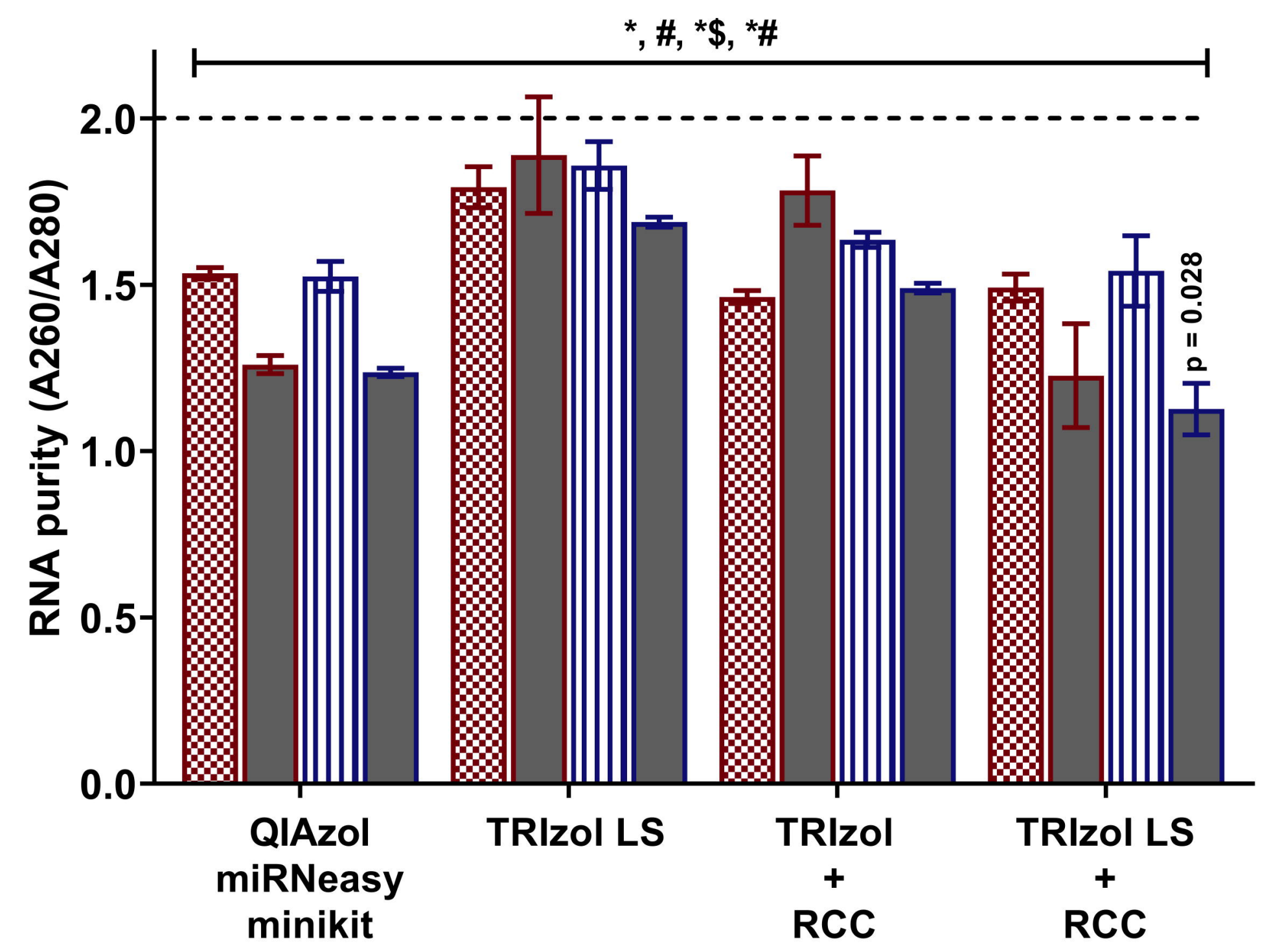

B

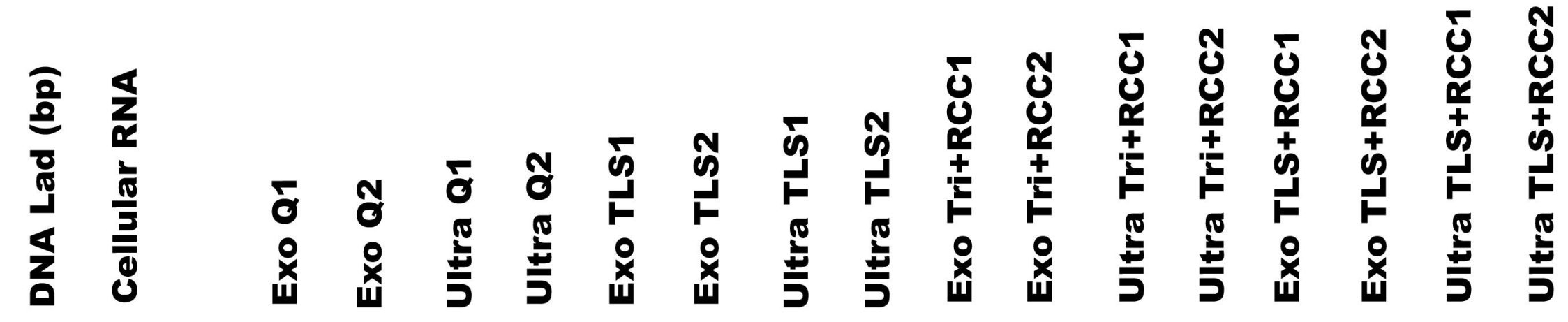

\begin{tabular}{|r|}
\hline $\mathbf{4}, 000$ \\
\hline 3,000 \\
2,500 \\
$\mathbf{2 , 0 0 0}$ \\
$\mathbf{1 , 5 0 0}$ \\
$\mathbf{1 , 0 0 0}$ \\
$\mathbf{7 5 0}$ \\
$\mathbf{5 0 0}$ \\
$\mathbf{2 5 0}$
\end{tabular}

Pellet

4,000

리

(5)
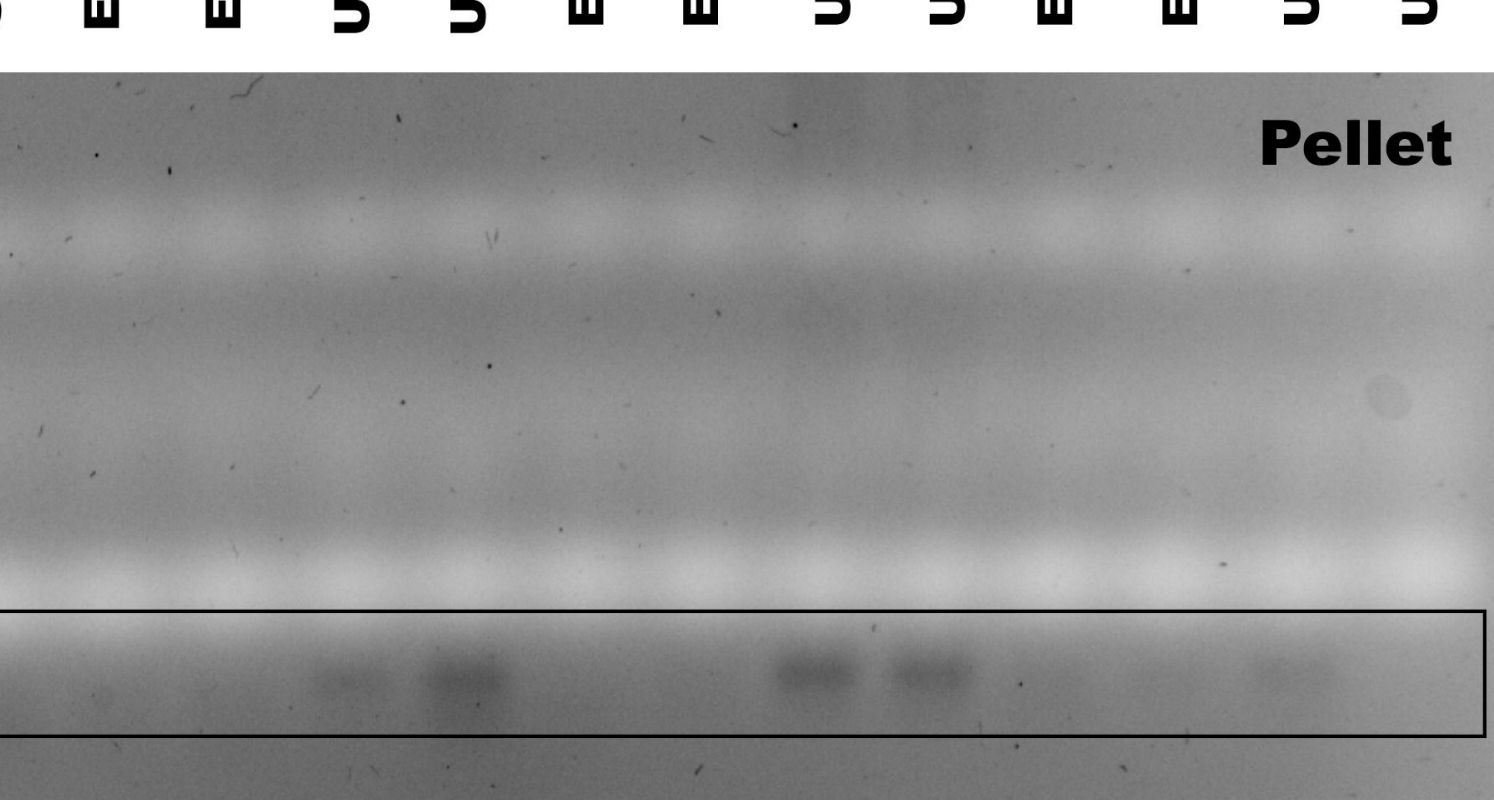

Supernatant

$\mathbf{2}, 500$
$\mathbf{1}, \mathbf{5 0 0}$
$\mathbf{1 , 0 0 0}$
$\mathbf{7 5 0}$

500

250

D

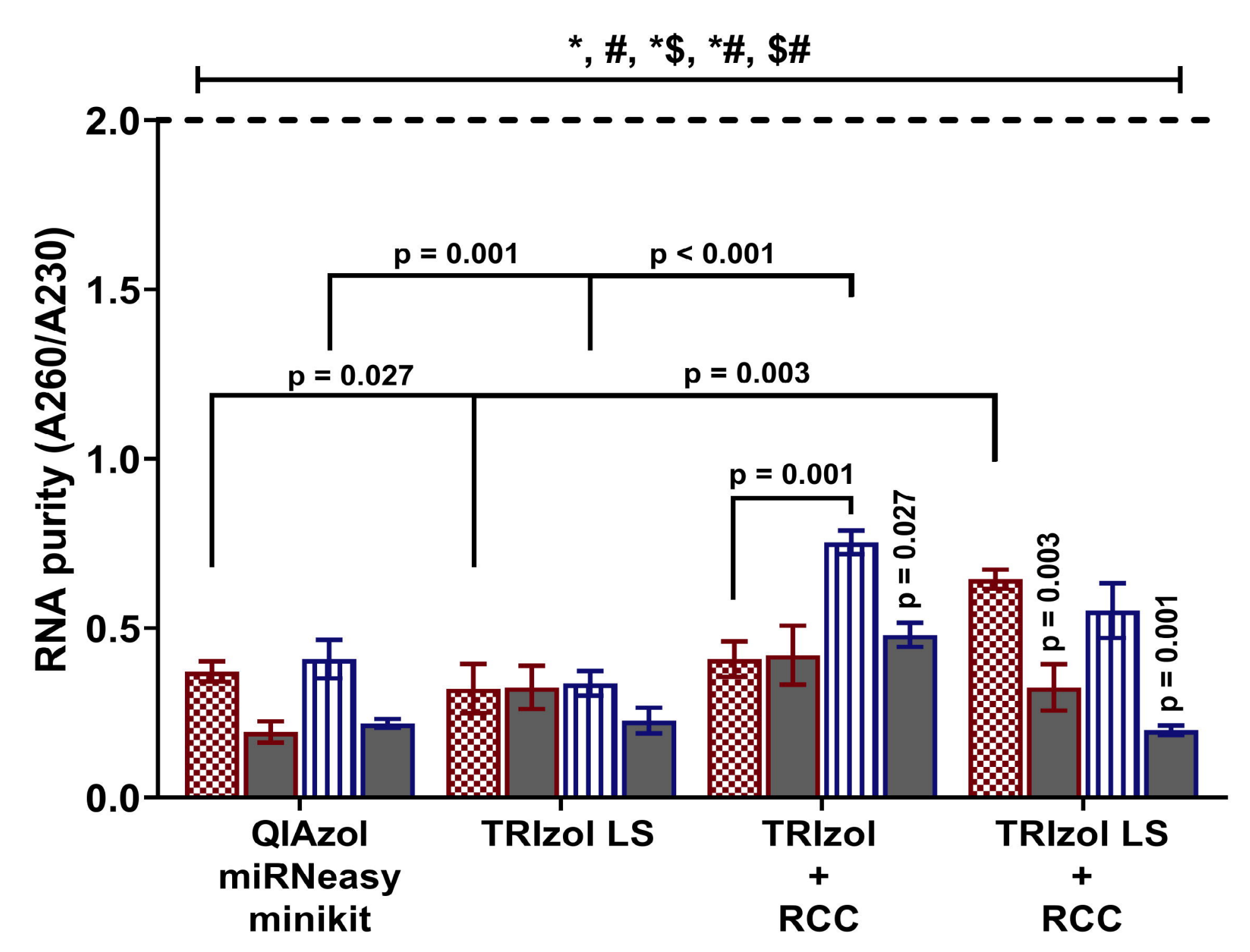

IIII Ultra Pellet

ExoQuick Supernatant 


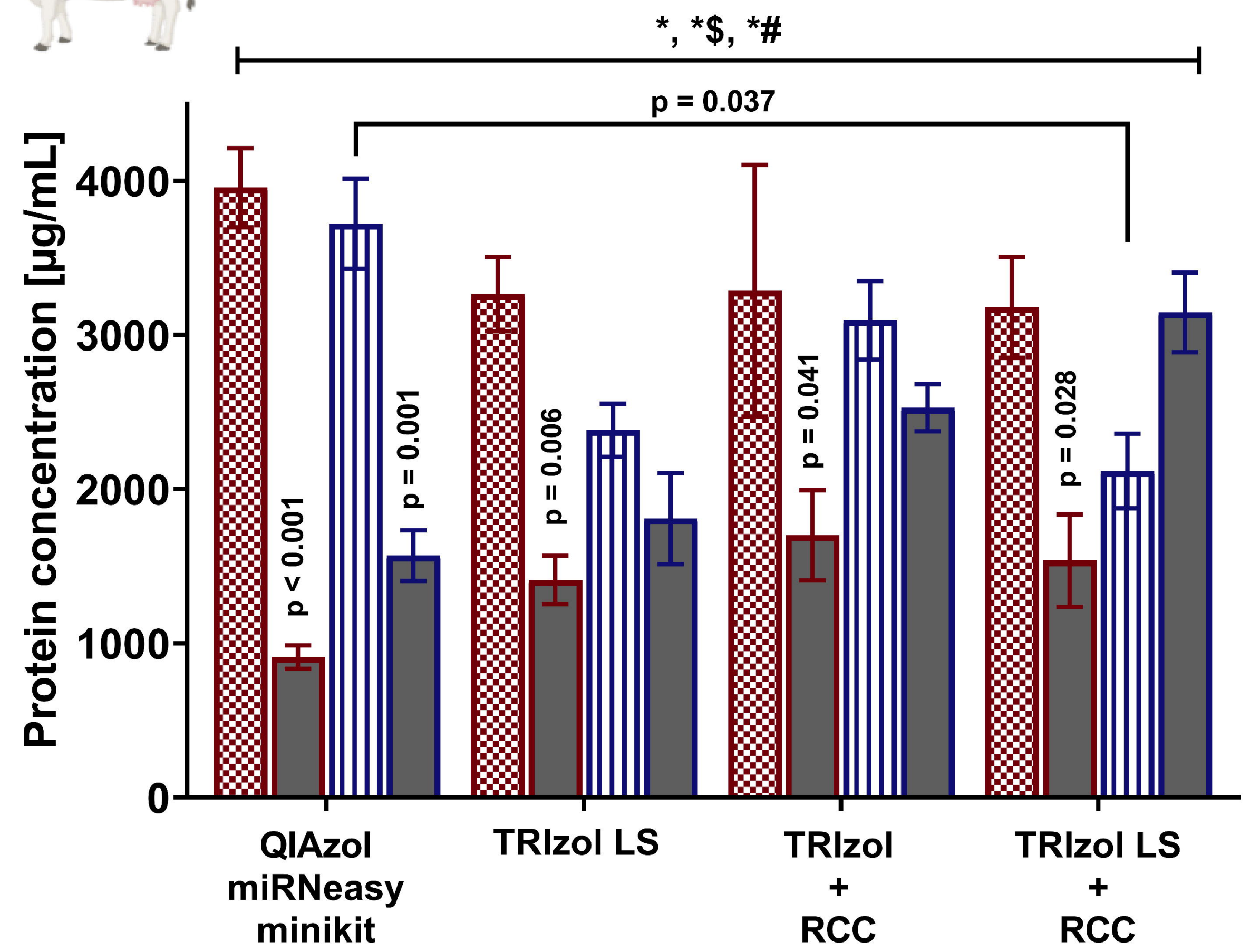

\%) ExoQuick Pellet

IIII Ultra Pellet

$\square$ ExoQuick Supernatant $\square$ Ultra Supernatant 
ExoQuick

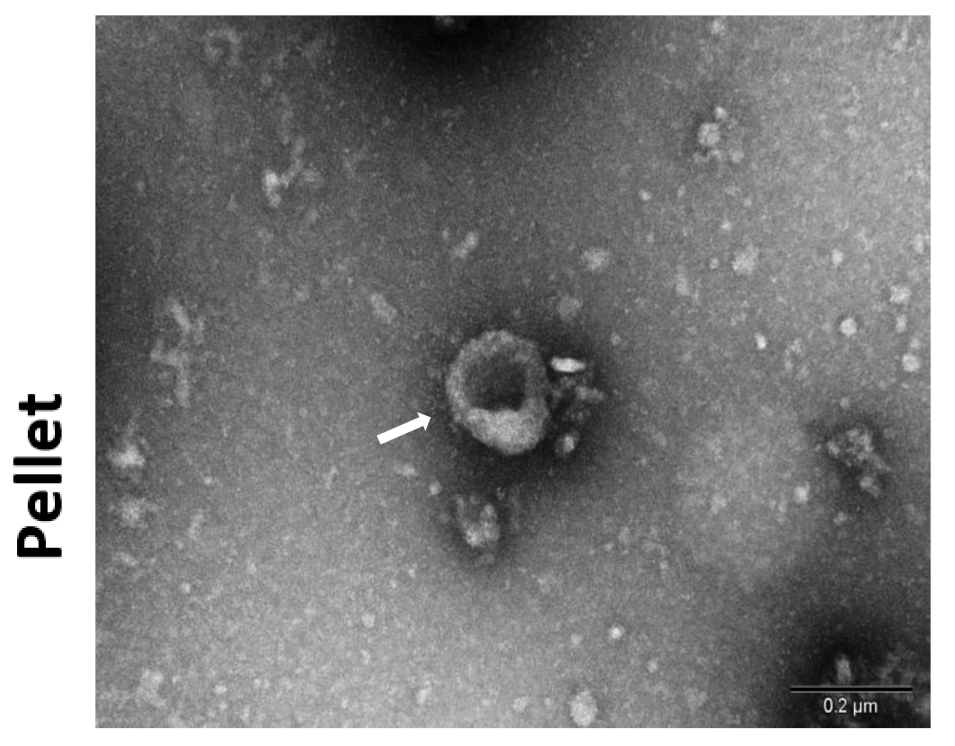

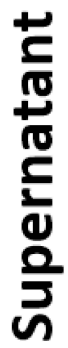

Ultracentrifugation
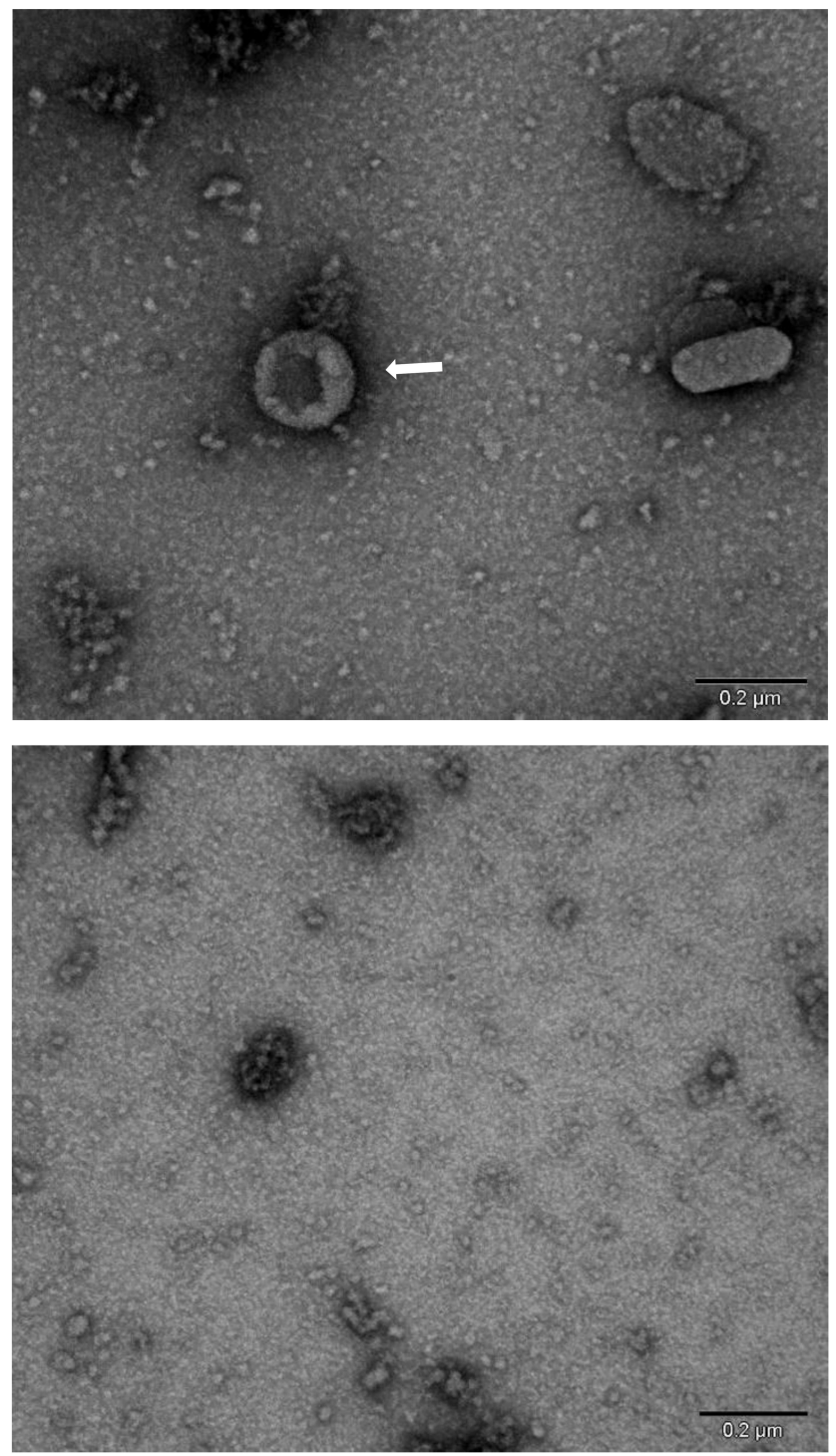
Exosome Concentration [Particles $/ \mathbf{m L}$ ]

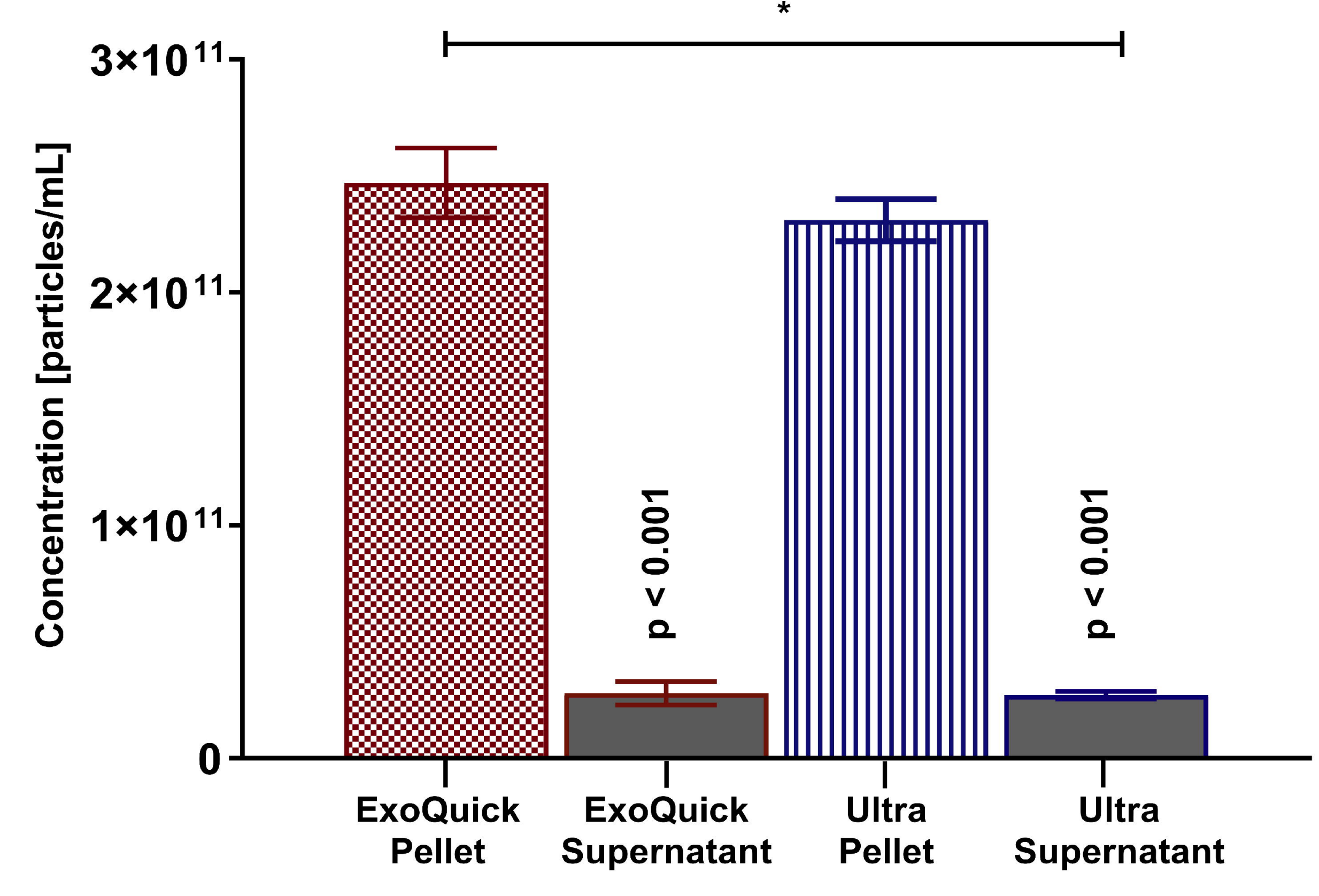

ExoQuick Pellet

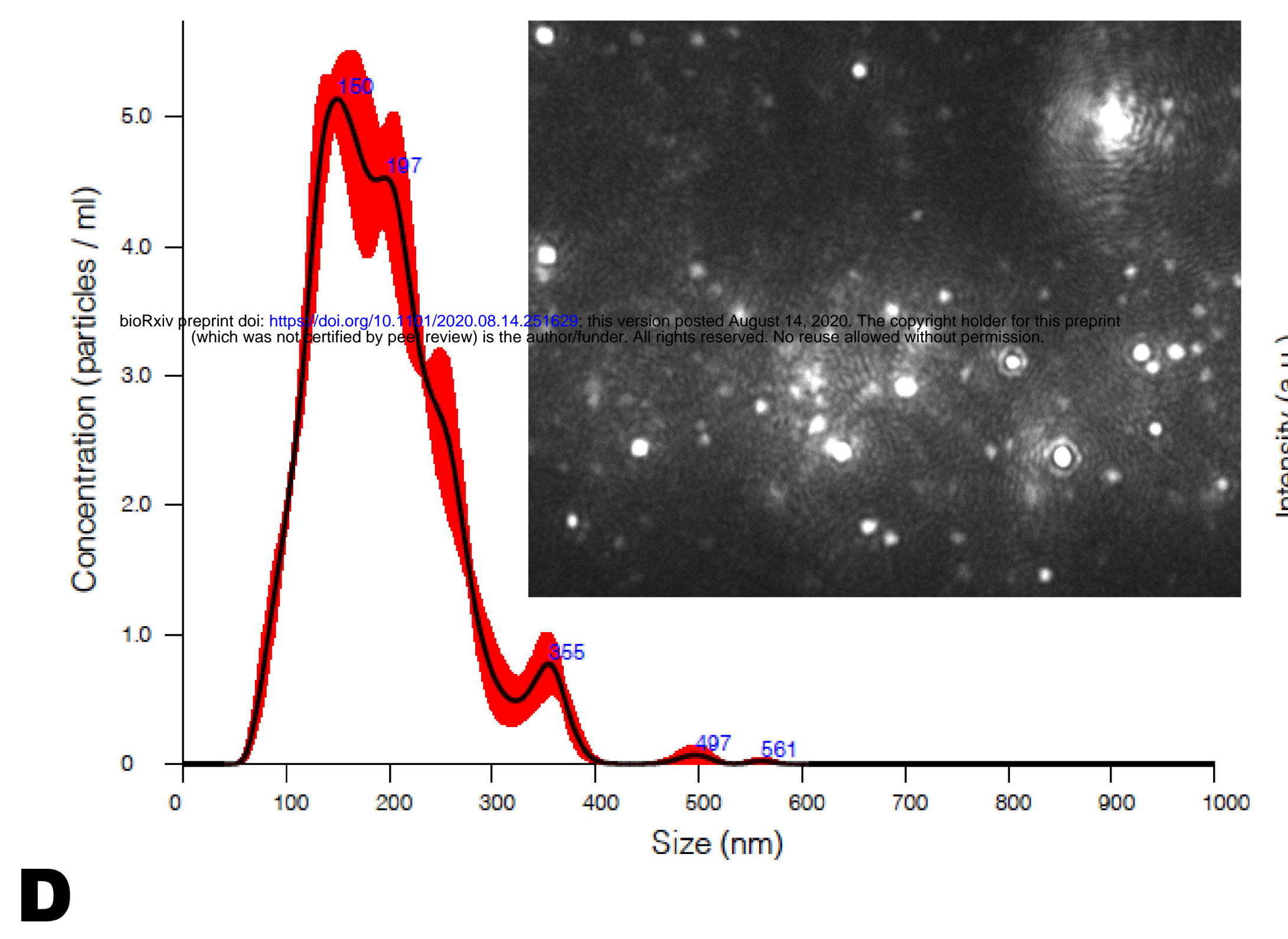

D

\section{ExoQuick Supernatant}

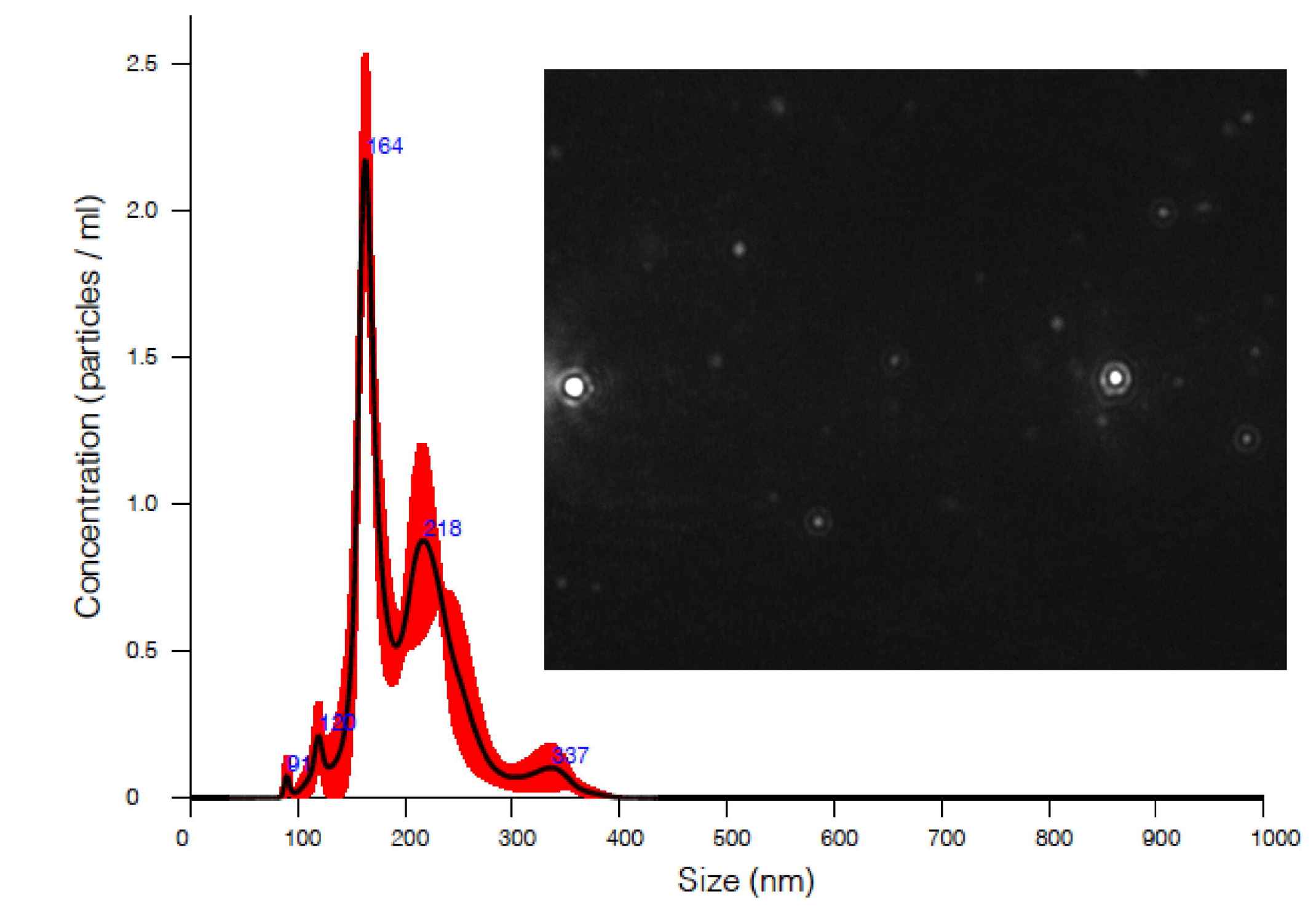

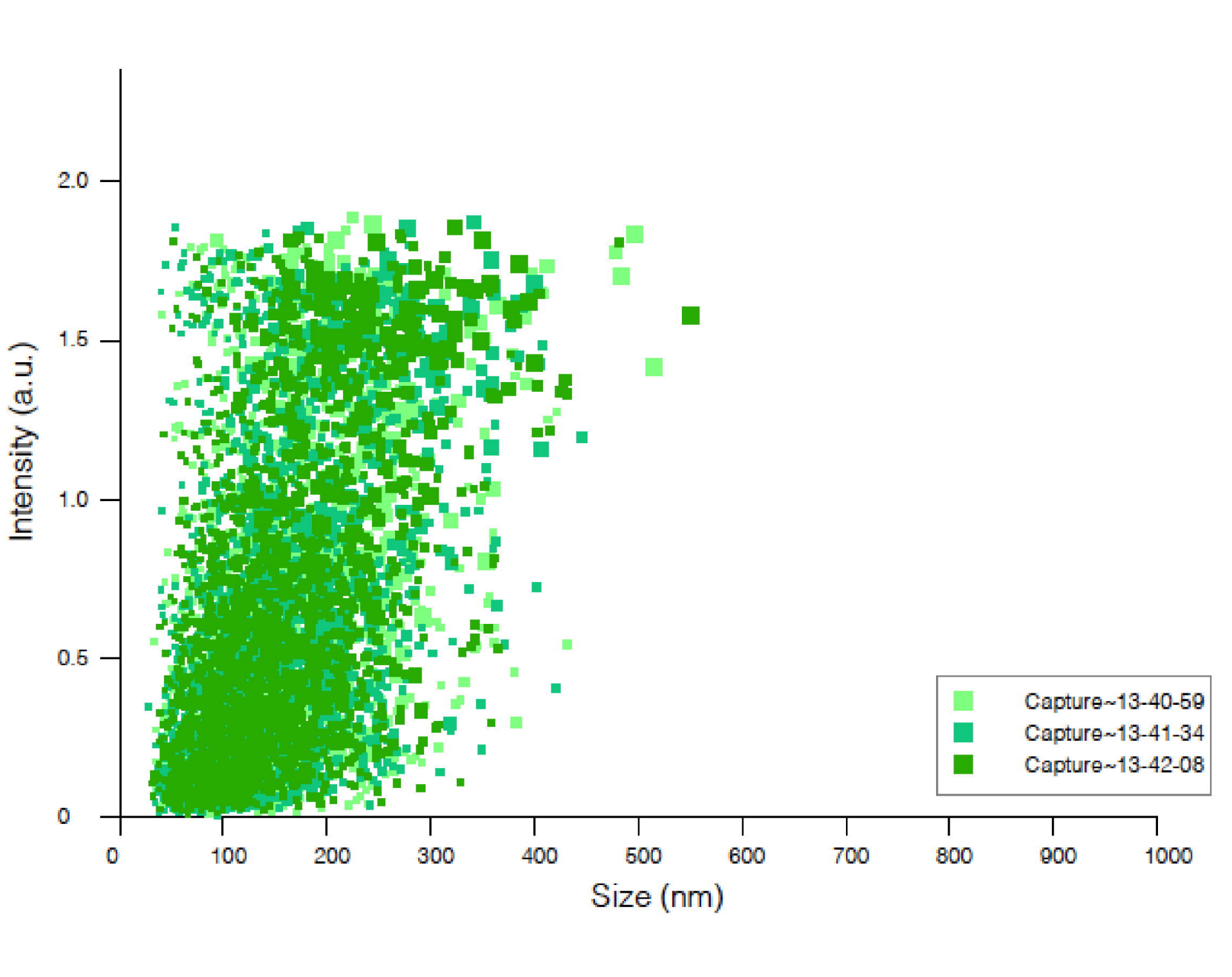

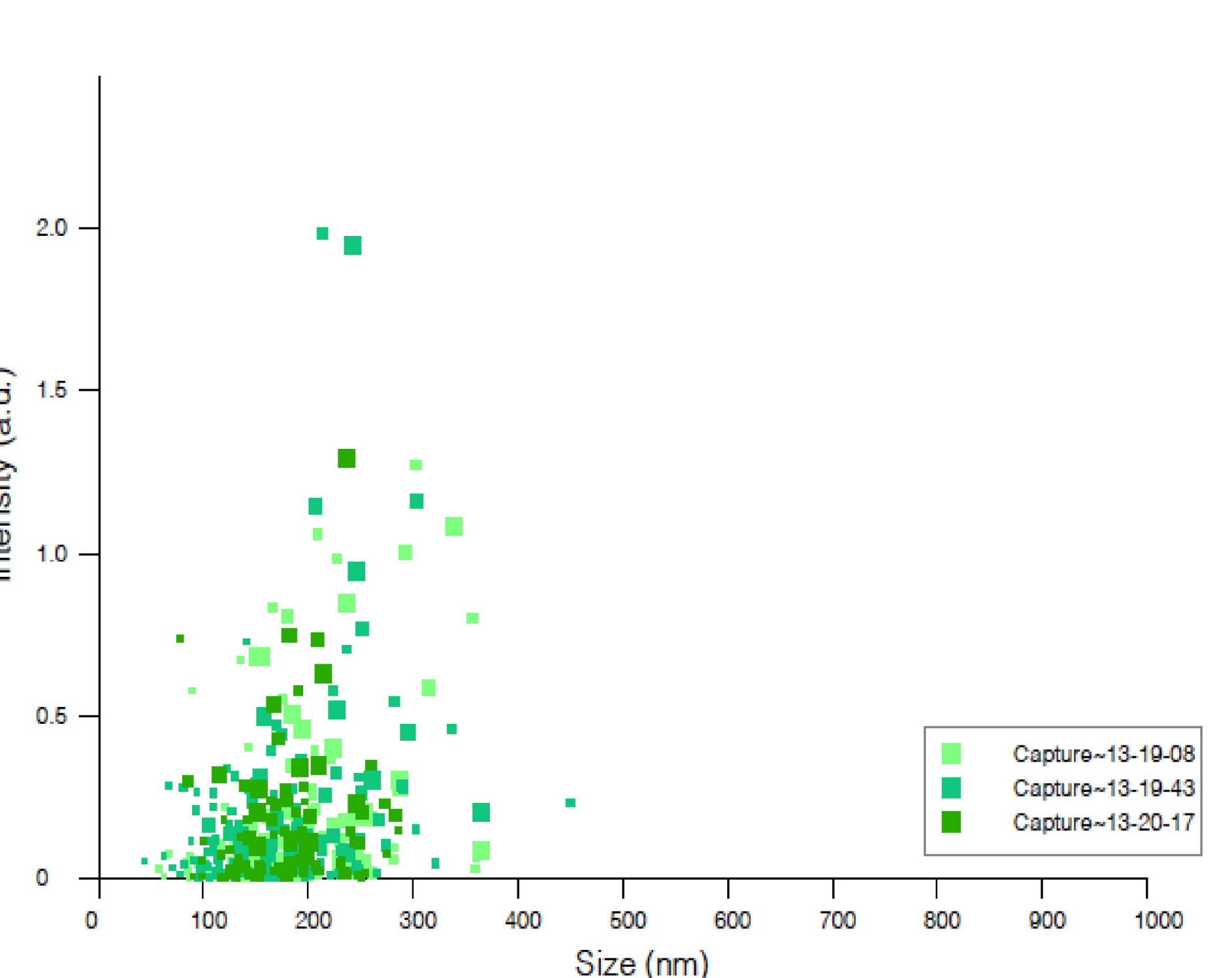

PBS Negative Control [Particles/mL]

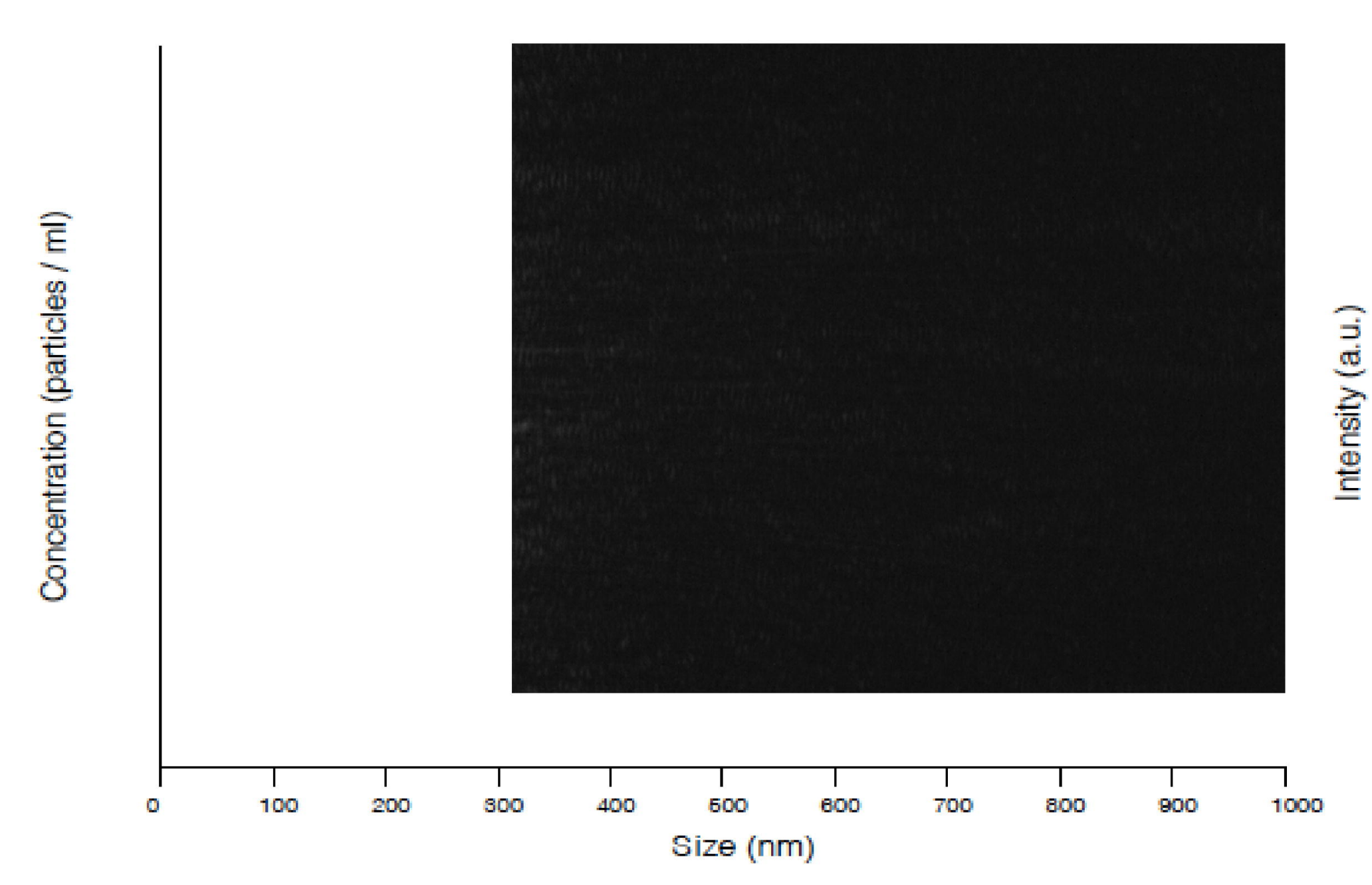

E

\section{Ultra Pellet}
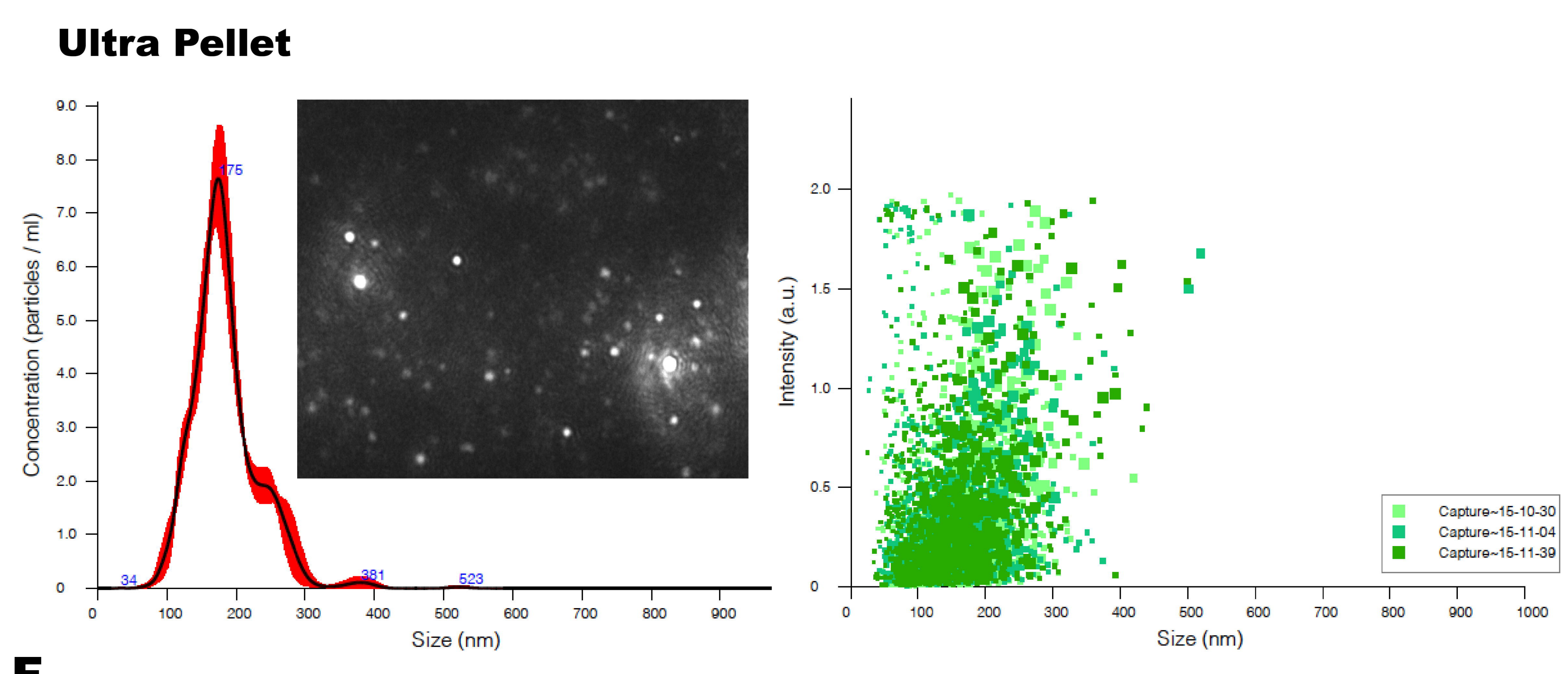

F

Ultra Supernatant

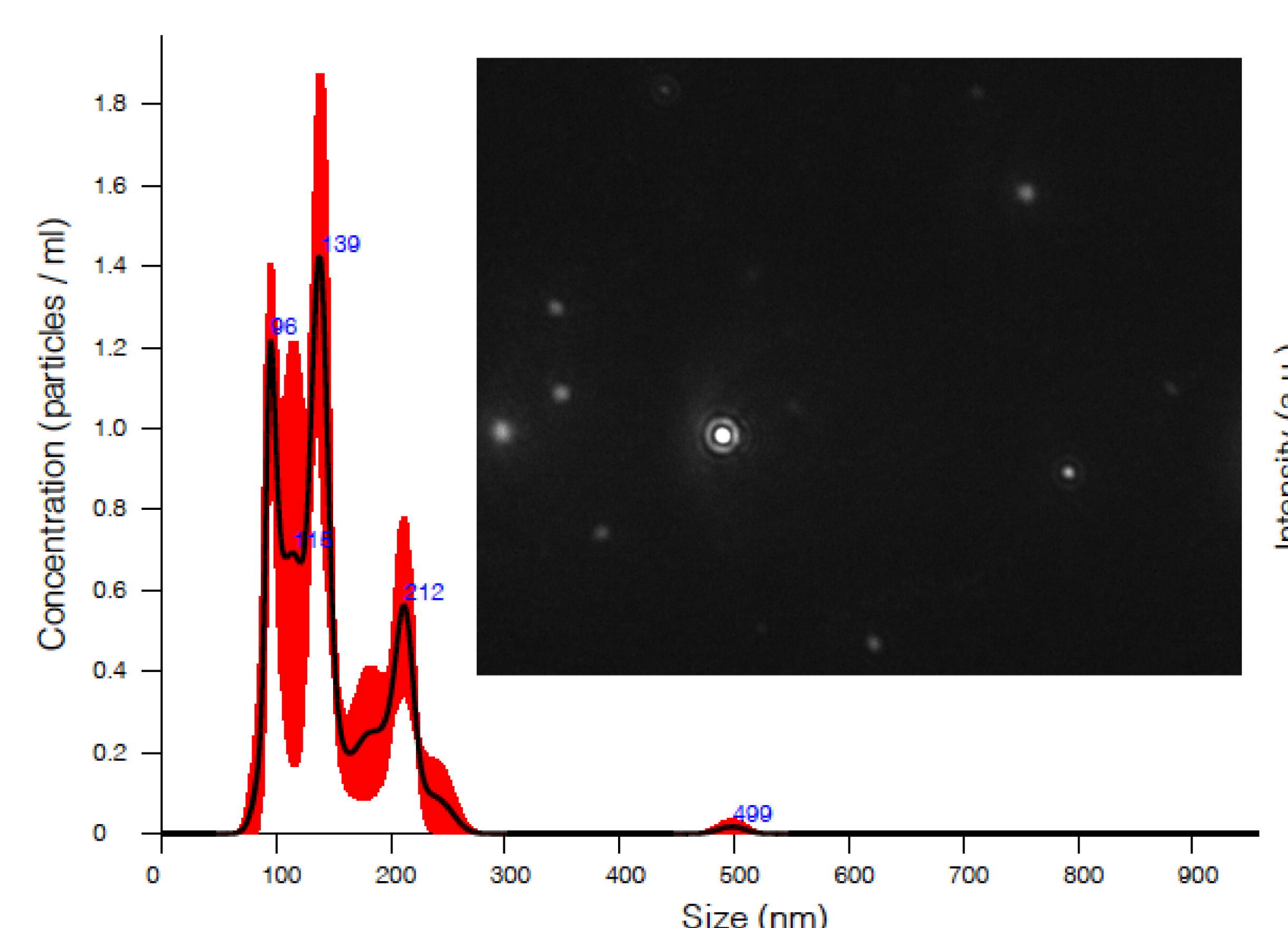

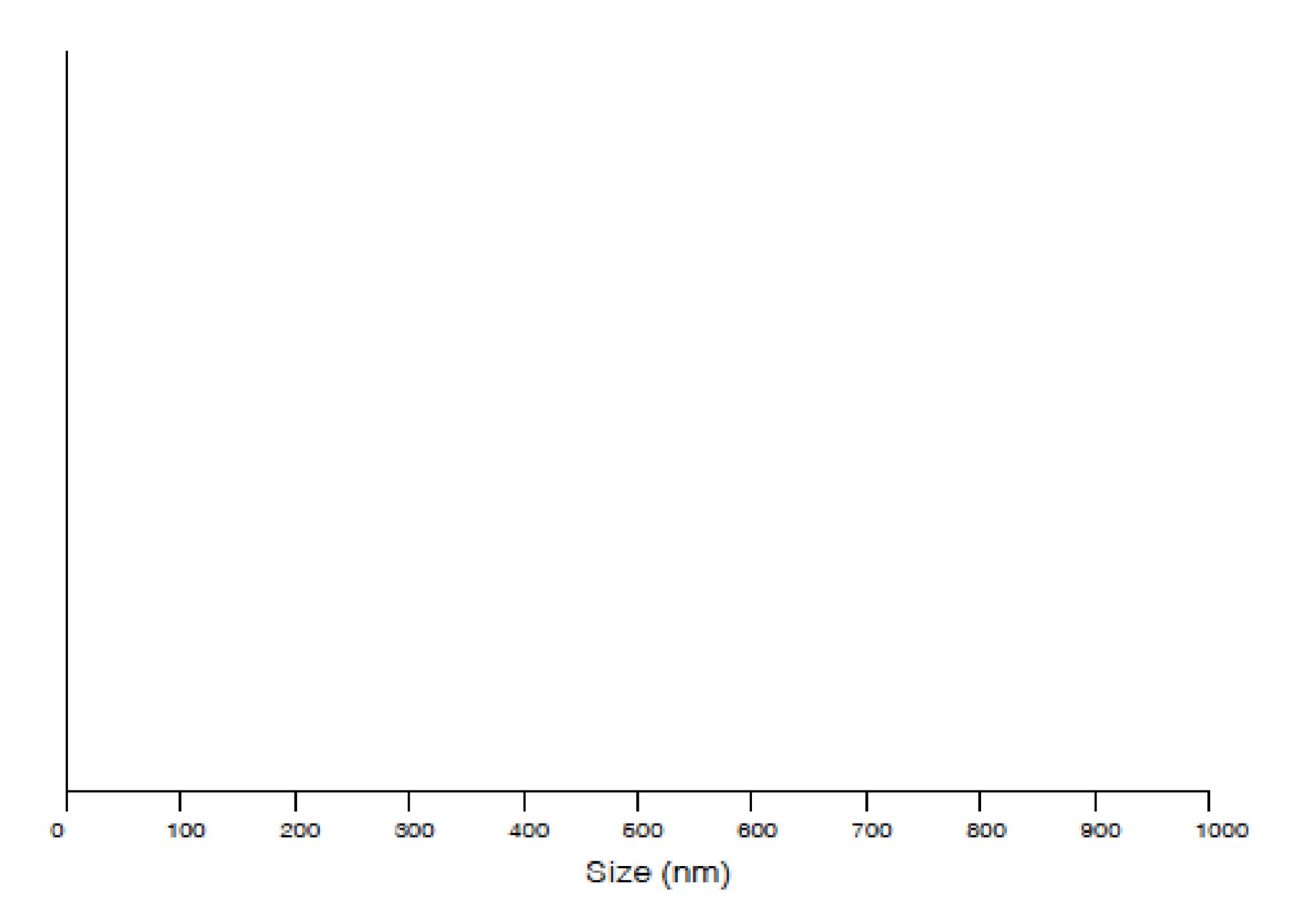

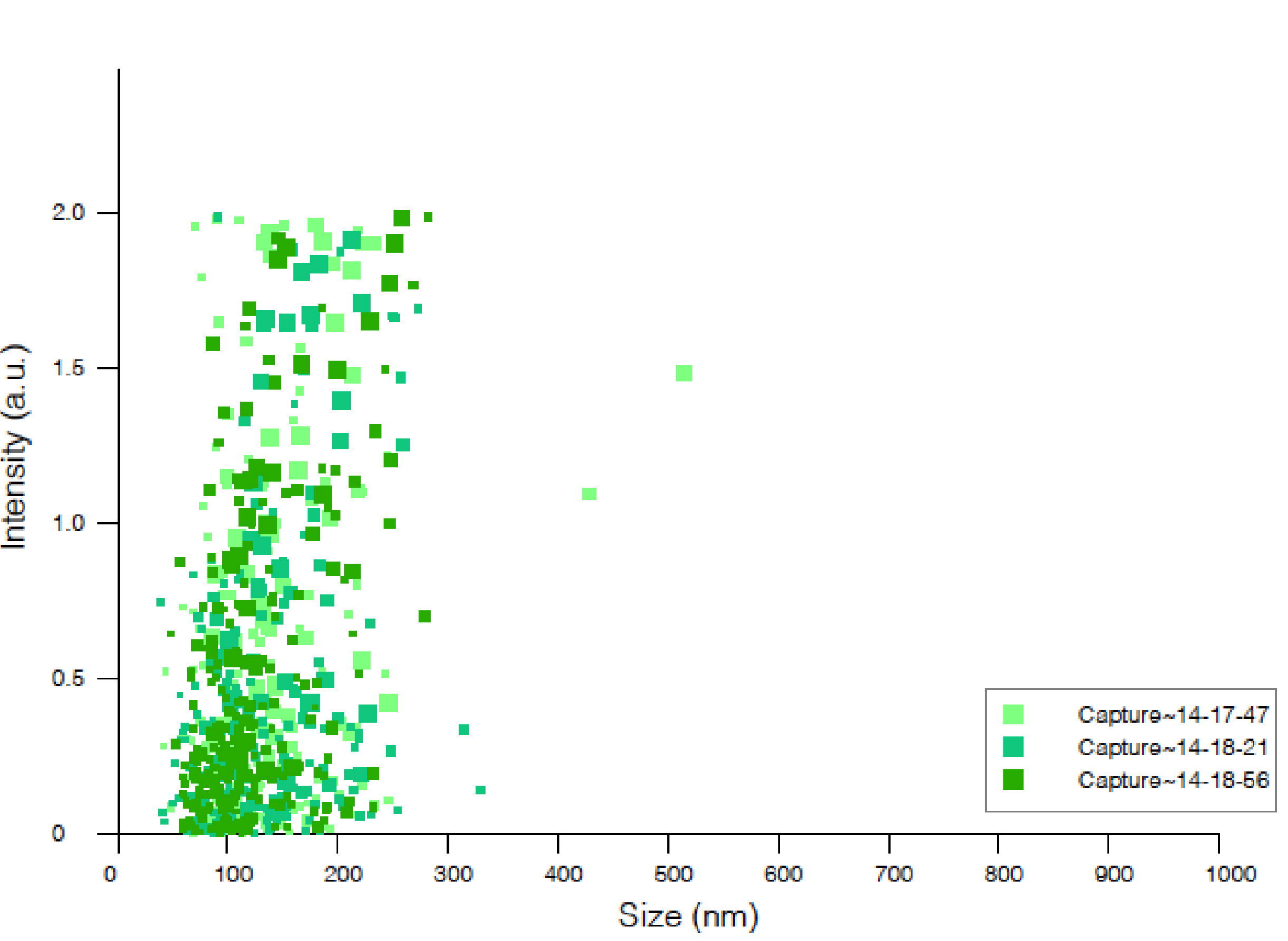


A
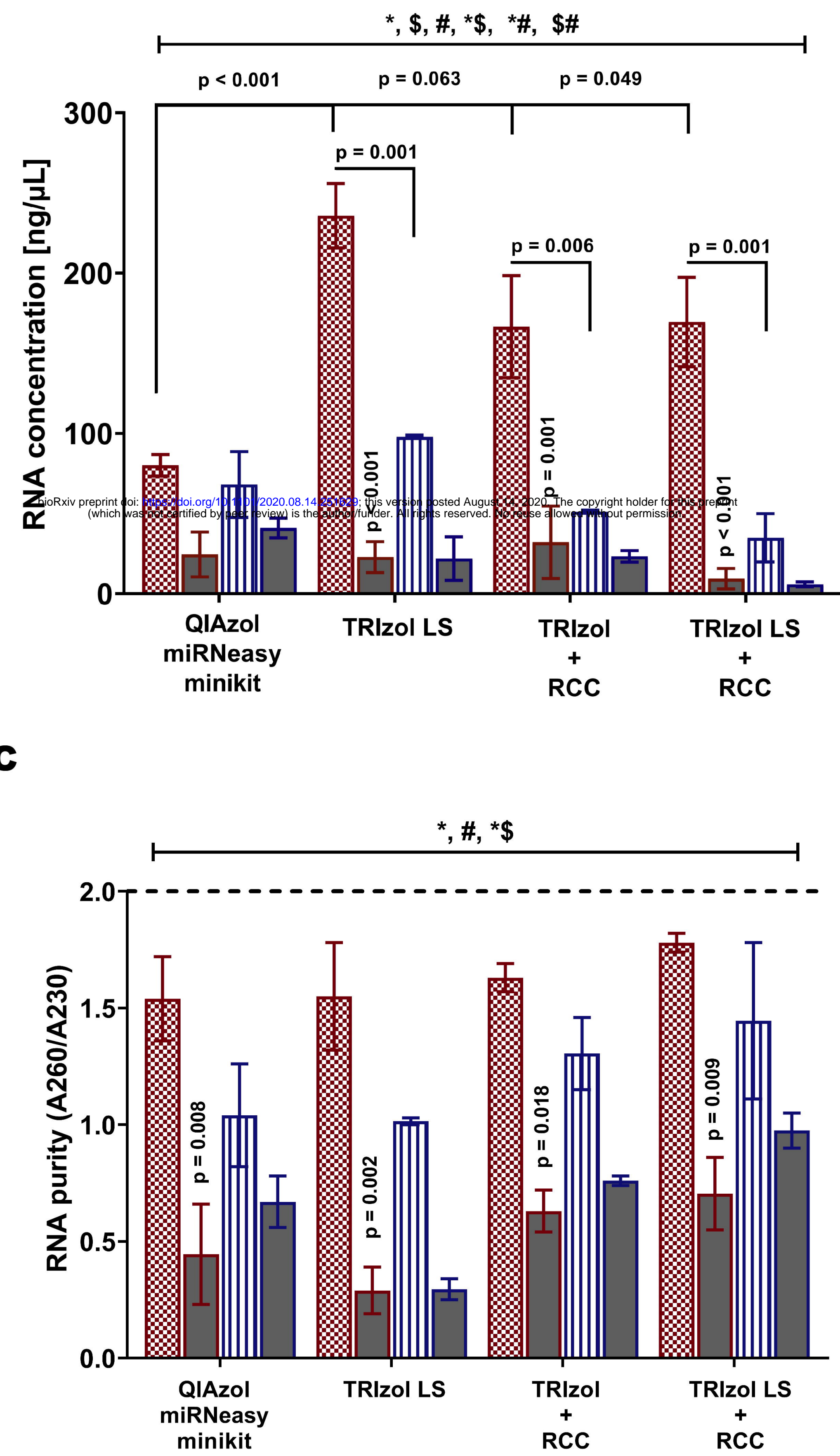

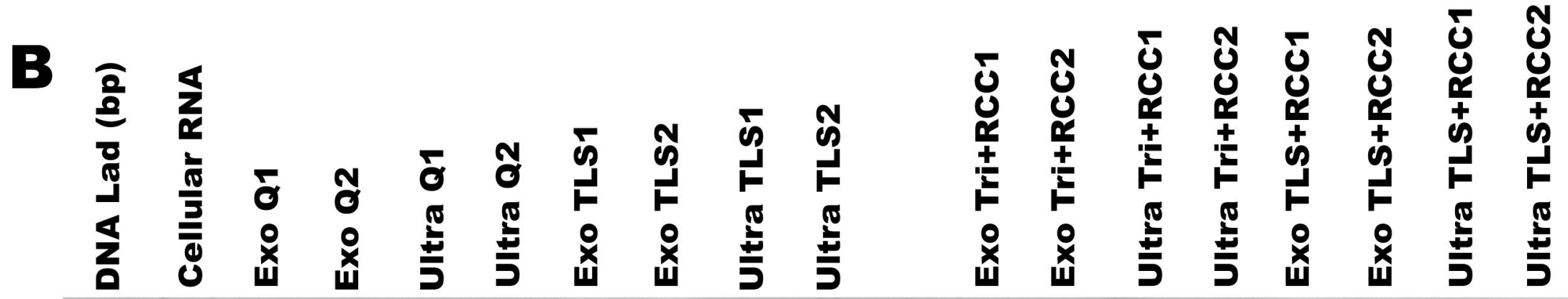

Pellet

Supernatant
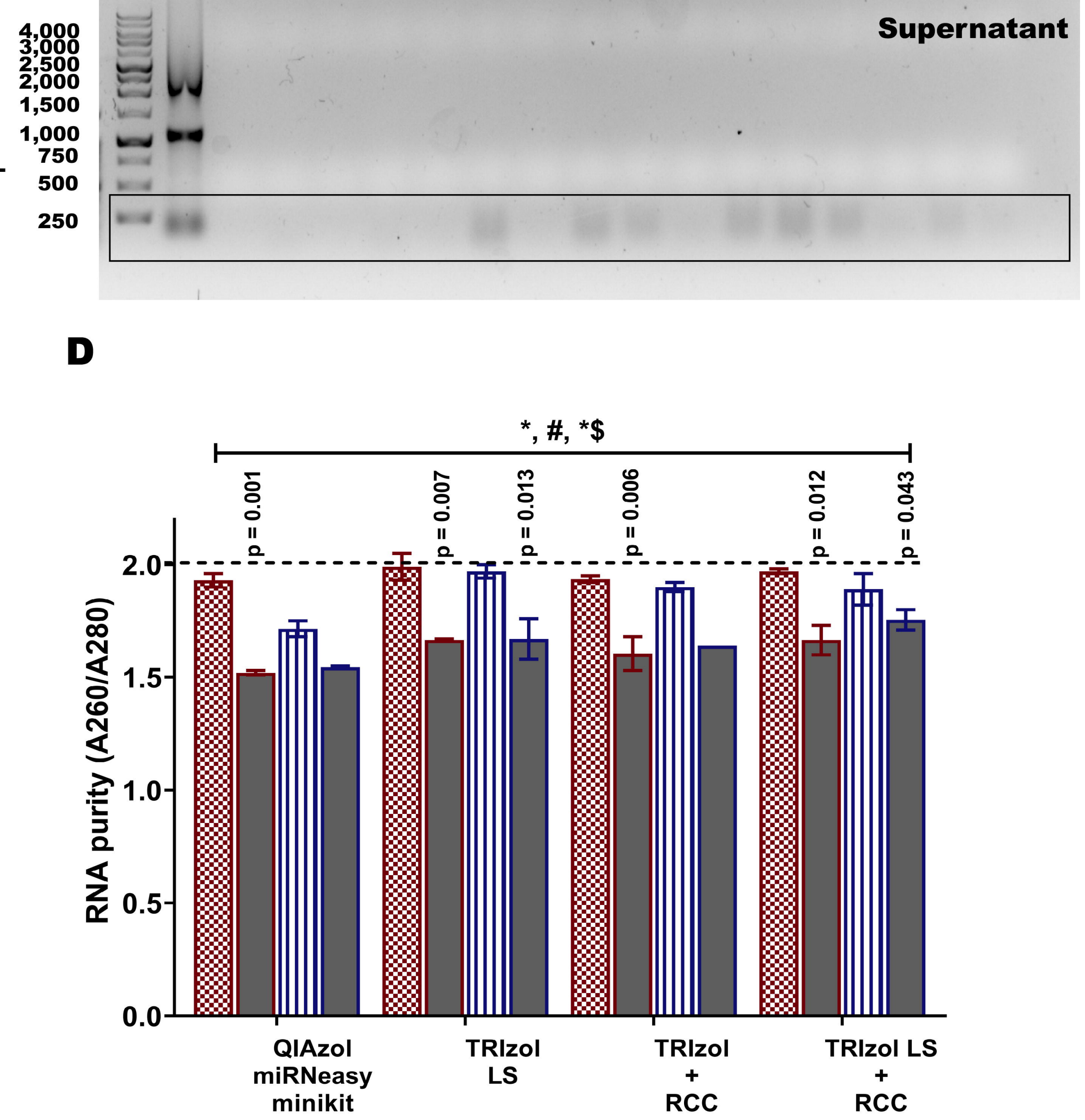

|l|I Ultra Pellet Ultra Supernatant 


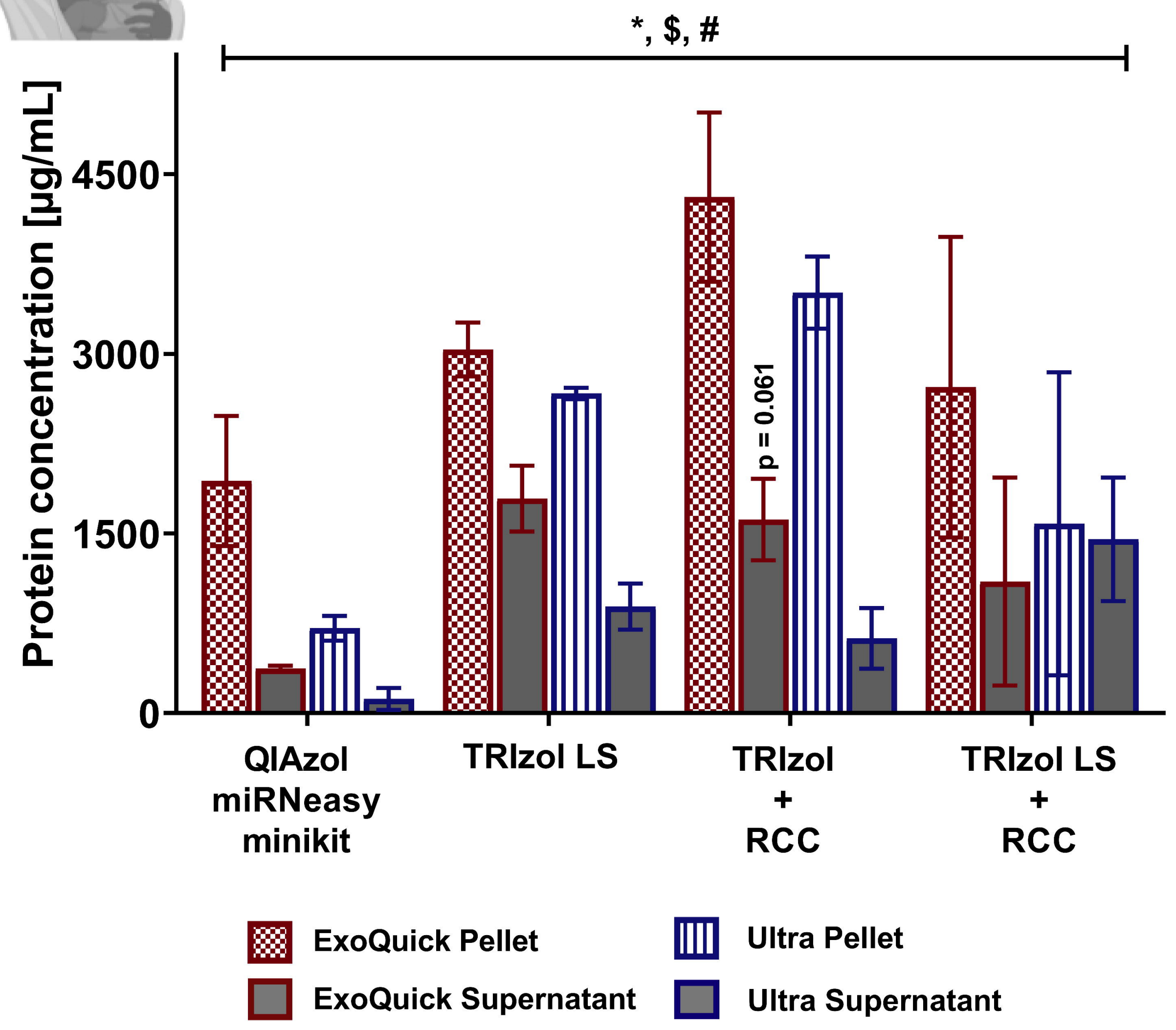

